\title{
Estabilidade assintótica e estrutural de campos vetoriais
}

\author{
Benito Frazão Pires
}




\title{
Estabilidade assintótica e estrutural de campos vetoriais.
}

\author{
Benito FrazÃo Pires*
}

Orientador: Prof. Dr. CARlos TeObaldo Gutierrez Vidalon

Tese apresentada ao Instituto de Ciências Matemáticas e de Computação da Universidade de São Paulo, ICMC-USP, como parte dos requisitos para obtenção do título de Doutor em Ciências-Área Matemática.

\author{
USP - São Carlos \\ Julho/2006 \\ (versão revisada)
}

${ }^{*}$ Este trabalho teve suporte financeiro da Fapesp e da Capes 
À minha família:

Meus pais, minhas irmãs, Ana Paula $\triangleright$ Bebezinho. 


\section{Agradecimentos}

Gostaria de externar meus agradecimentos a todos aqueles que contribuíram para a realização deste trabalho, sobretudo...

Ao Prof. Carlos Gutierrez pela presteza e perspicácia sempre presentes em sua orientação; e sobretudo por ter tornado esta jornada pelos sistemas dinâmicos, desde os meus primeiros passos, muito motivante e prazerosa.

Aos meus pais e às minhãs irmãs, presença constante em todos os momentos.

À Ana Paula pela paciência e compreensão, sobretudo nesta etapa final.

Aos apoios financeiros da FAPESP, durante o meu doutorado no Brasil, e da CAPES, durante o meu estágio de doutoramento na Universidade Autônoma de Barcelona, sem os quais este trabalho não teria tido êxito.

Ao Prof. Carlos Biasi, pelas brilhantes aulas de matemática, com as quais aprendi muito.

Ao Prof. Jaume Llibre por ter supervisionado meu estágio de doutoramento na UAB e ao Prof. Armengol Gasull pelo interessante curso de Teoria de Bifurcações.

A todos os meus amigos, sobretudo àqueles que sempre me incentivaram a trilhar este caminho.

A todos os funcionários da USP e da UAB pela presteza e prontidão. 


\section{Resumo}

O objetivo deste trabalho é provar um Closing Lema Parcial para variedades bidimensionais compactas, orientáveis ou não-orientáveis. Para enunciá-lo, considere um campo vetorial

$X \in \mathfrak{X}^{r}(M), r \geq 2$, de classe $C^{r}$ em uma variedade bidimensional compacta $M$, e seja $\Sigma$ um segmento transversal a $X$ passando por um ponto recorrente não-trivial $p$ de $X$. Seja $P: \Sigma \rightarrow \Sigma$ a correspondente transformação de primeiro retorno. O primeiro resultado deste trabalho consiste em mostrar que se $P$ tem a propriedade de que para todo $n \geq N$ e $x \in \operatorname{dom}\left(P^{n}\right),\left|D P^{n}(x)\right|<\lambda$, onde $N \in \mathbb{N}$ e $0<\lambda<1$, então existe um campo vetorial $Y$ arbitrariamente próximo de $X$ na topologia $C^{r}$ tendo uma trajetória periódica passando por $p$. O segundo resultado consiste em apresentar condições, sobre os expoentes de Lyapunov de $P$, para que $\left|D P^{n}\right|<\lambda$ para todo $n \geq N$. Nesta tese, também incluímos um resultado sobre a estabilidade assintótica no infinito de campos planares diferenciáveis, mas não necessariamente de classe $C^{1}$. 


\begin{abstract}
The aim of this work is to provide a Partial $C^{r}$ Closing Lemma for compact surfaces, orientable or non-orientable. To state it, let $X \in \mathfrak{X}^{r}(M), r \geq 2$, be a $C^{r}$ vector field on a compact surface $M$ and let $\Sigma$ be a transverse segment to $X$ passing through a non-trivial recurrent point $p$ of $X$. Let $P: \Sigma \rightarrow \Sigma$ be the corresponding first return map. The first result of this work consists in showing that if $P^{n}$ has the property that for all $n \geq N$ and $x \in \operatorname{dom}\left(P^{n}\right),\left|D P^{n}(x)\right|<\lambda$, where $N \in \mathbb{N}$ e $0<\lambda<1$, then there exists a vector field $Y$ arbitrarily close to $X$ in the $C^{r}$ topology such that $p$ is a periodic point of $Y$. The second result consists in presenting sufficient conditions, upon the Lyapunov exponents of $P$, so that $\left|D P^{n}\right|<\lambda$ for all $n \geq N$. In this thesis, we also include a result concerning the asymptotic stability at infinity of planar differentiable vector fields, not necessarily of class $C^{1}$.
\end{abstract}




\section{Sumário}

$\begin{array}{ll}\text { Introdução } & 1\end{array}$

1 Connecting Lema e Closing Lema 9

1.1 Introdução . . . . . . . . . . . . . . . . . . 9

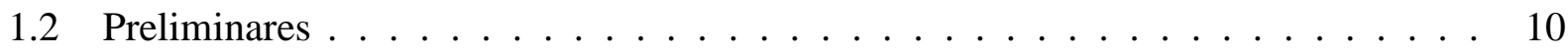

1.3 Fluxos em Superfícies . . . . . . . . . . . . . . . . . 13

1.4 Tranformações de Primeiro Retorno Contrativas . . . . . . . . . . . . . . . . . 21

1.5 Connecting Lema . . . . . . . . . . . . . . . . . . . 25

1.6 Closing Lema . . . . . . . . . . . . . . . . . . . . 30

1.7 Medida Transversal e Transformações de Primeiro Retorno . . . . . . . . . . . . . 32

1.8 Sobre o divergente de um campo vetorial nas suas selas . . . . . . . . . . 33

1.9 O caso unicamente ergódico . . . . . . . . . . . . . . . . . 34

2 Estabilidade Assintótica no Infinito para Campos Vetoriais Diferenciáveis 41

2.1 Introdução . . . . . . . . . . . . . . . . . . . 41

2.2 Campos Vetoriais Diferenciáveis . . . . . . . . . . . . . . 43

2.3 Seções Transversais a Campos Vetoriais Contínuos . . . . . . . . . . . . . . 49

2.4 Pseudo Setor Hiperbólico no Infinito . . . . . . . . . . . . . . . . 50 
2.5 Círculos Transversais ao Redor do Infinito . . . . . . . . . . . . . . . . . . 54

2.6 Estabilidade Assintótica no Infinito . . . . . . . . . . . . . . . . . . 57 


\section{Introdução}

O presente trabalho aborda dois problemas que podem ser inseridos dentro do contexto da dinâmica bidimensional. O primeiro atende pelas denominações genéricas "Connecting Lema" e "Closing Lema" para fluxos. O segundo problema consiste em estudar a estabilidade assintótica ao redor do infinito de campos vetoriais planares diferenciáveis, mas não necessariamente de classe $C^{1}$.

De maneira informal, o primeiro problema consiste em saber se uma trajetória recorrente nãotrivial de um fluxo em uma variedade bidimensional compacta pode ser fechada por perturbações apropriadas e arbitrariamente pequenas do fluxo original. O segundo problema consiste em determinar sob que condições as trajetórias de um campo vetorial planar diferenciável vão (resp. vêm) do infinito no sentido de que elas cruzam todos os círculos centrados na origem com diâmetros suficientemente grandes.

Passemos agora a discutir o primeiro problema. O problema aberto conhecido como "Closing Lema de classe $C^{r}$ " pode ser enunciado da seguinte maneira:

Problema 1 (Closing Lema de classe $C^{r}$ ) Sejam $M$ uma variedade suave compacta, $r \geq 2$ um número inteiro, $X \in \mathfrak{X}^{r}(M)$ um campo vetorial de classe $C^{r}$ em $M$, e p um ponto não-errante de $X$. Existe $Y \in \mathfrak{X}^{r}(M)$ arbitrariamente próximo de $X$ na topologia $C^{r}$ tal que $p$ seja um ponto periódico de $Y$ ?

Um versão local deste problema é a seguinte:

Problema 2 (Closing Lema de classe $C^{r}$ localizado) Sejam $M$ uma variedade suave compacta, $r \geq 2$ um número inteiro, $X \in \mathfrak{X}^{r}(M)$ um campo vetorial de classe $C^{r}$ em $M$, e $p$ um ponto nãoerrante de $X$. Para cada vizinhança $V$ de $p$, e para cada vizinhança $\mathscr{V}$ de $X$ em $\mathfrak{X}^{r}(M)$, existe $Y \in \mathscr{V}$ que é uma perturbação de $X$ suportada em $V$ tal que $Y$ tenha uma trajetória periódica passando por $V$ ? 
É óbvio que se o Problema 2 tiver uma resposta positiva para uma classe de campos vetoriais $\mathscr{N} \subset \mathfrak{X}^{r}(M)$, então o mesmo será verdade para o Problema 1 , com relação à mesma classe $\mathscr{N}$. Foi provado por Pugh [54] que o Teorema 2 é verdadeiro em classe $C^{1}$, enquanto que há contraexemplos em classe $C^{2}$ (ver [22]).

Vamos nos restringir aos campos vetoriais sobre variedades bidimensionais compactas. Por variedade bidimensional compacta entendemos uma variedade conexa, compacta, sem fronteira e de classe $C^{\infty}$. Neste caso, no enunciado do Closing Lema (Problema 1) podemos assumir que $p$ é um ponto recorrente não-trivial [51]. Nosso objetivo é provar um Closing Lema de classe $C^{r}$ para uma classe de fluxos em variedades bidimensionais compactas, orientáveis ou não, admitindo recorrência não-trivial. Relembramos que a esfera $\mathbb{S}^{2}$, o plano projetivo $\mathbb{P}^{2}$, e a garrafa de Klein $\mathbb{K}^{2}$ [42] não admitem fluxos com recorrência não-trivial. Aparte destes casos, todas as outras variedades bidimensionais compactas admitem fluxos com recorrência não-trivial [38]. Além disso, sabe-se que a única superfície admitindo fluxos sem singularidades e com recorrência não-trivial é o toro $\mathbb{T}^{2}$. Quando $M=\mathbb{T}^{2}$ e o campo vetorial não tem singularidades, Peixoto [52] provou que $C^{r}$-closing vale. Assim iremos considerar somente campos vetoriais tendo pelo menos uma singularidade.

Como uma etapa intermediária na direção do nosso Closing Lema de classe $C^{r}$, vamos dar uma resposta positiva parcial à seguinte versão do Connecting Lema de classe $C^{r}$ :

Problema 3 (Connecting Lema de classe $C^{r}$ fraco) Sejam M uma variedade bidimensional compacta e $X \in \mathfrak{X}^{r}(M), r \geq 1$, um campo vetorial que tem singularidades, todas hiperbólicas e uma trajetória recorrente não-trivial. Existe $Y \in \mathfrak{X}^{r}(M)$ arbitrariamente próximo de $X$ na topologia $C^{r}$ tendo uma conexão de sela a mais que $X$ ?

Antes de enunciar nossos resultados, vamos dar algumas definições.

Seja $X \in \mathfrak{X}^{r}(M)$ um campo vetorial de classe $C^{r}$ que tem uma trajetória recorrente não-trivial $\gamma$ e sejam $\Sigma_{1}, \Sigma_{2}$ segmentos transversais (resp. círculos transversais) a $X$ passando por $\gamma$. A aplicação de Poincaré induzida por $X$ em $\Sigma_{1}, \Sigma_{2}$ é a aplicação, não necessariamente definida em todos os pontos, que leva o ponto $p \in \Sigma_{1}$ na primeira intersecção de $\gamma_{p}^{+}-\{p\} \Sigma_{2}$, onde $\gamma_{p}^{+}$denota a semitrajetória positiva de $X$ iniciando-se em $p$. No caso em que $\Sigma_{1}=\Sigma_{2}=\Sigma$, denominamos a aplicação $P$ a transformação de primeiro retorno induzida por $X$ em $\Sigma$ (ou simplesmente a correspondente transformação de primeiro retorno). Referimo-nos a estas aplicações como os isomorfismos canônicos. Com relação a aplicações de primeiro retorno $P$ induzidas em segmentos 
transversais $\Sigma$, por convenção, vamos remover do domínio de $P$ aqueles pontos que são levados por $P$ nos extremos de $\Sigma$ (evitando assim pontos de descontinuidade dentro do domínio de $P$ ).

Seja $\Sigma$ um segmento transversal (resp. círculo transversal) a um campo vetorial $X \in \mathfrak{X}^{r}(M)$. A derivada $D P$ da correspondente transformação de primeiro retorno $P: \Sigma \rightarrow \Sigma$ induz canonicamente uma aplicação real $D P: \Sigma \rightarrow \mathbb{R}$. Estamos interessados naquelas aplicações de primeiro retorno $P$ cujo enésimo iterado $P^{n}$ é uma contração no sentido da seguinte definição.

Definição 0.0.1 ( $\lambda$-contração infinitesimal) Sejam $\Sigma$ um segmento transversal (resp. círculo transversal) a um campo vetorial de classe $C^{r}, P: \Sigma \rightarrow \Sigma$ a correspondente transformação de primeiro retorno e $0<\lambda<1$. Dado $n \in \mathbb{N}$, dizemos que $P^{n}$ é uma $\lambda$-contração infinitesimal se $\left|D P^{n}(x)\right|<\lambda$, para todo $x \in \operatorname{dom}\left(P^{n}\right)$.

Seja $X \in \mathfrak{X}^{r}(M)$ uma campo vetorial de classe $C^{r}$ que tem uma trajetória recorrente não-trivial $\gamma$ cujo fecho topológico denotamos por $\operatorname{cl}(\gamma)$. Dada uma vizinhança aberta $V$ de $\gamma$, seja $\left\{\Sigma_{\alpha}\right\}_{\alpha \in \mathscr{A}}$ a coleção de todos os segmentos transversais a $X$ passando por $\gamma$ e contidos em $V$. Uma medida transversal para $\gamma$ é uma tripla $\left(V,\left\{\Sigma_{\alpha}\right\}_{\alpha \in \mathscr{A}},\left\{v_{\alpha}\right\}_{\alpha \in \mathscr{A}}\right)$, onde $V$ e $\left\{\Sigma_{\alpha}\right\}_{\alpha \in \mathscr{A}}$ são como acima, e cada $v_{\alpha}$ é uma medida de probabilidade de Borel em $\Sigma_{\alpha}$, não-atômica, suportada em $\operatorname{cl}(\gamma) \cap \Sigma_{\alpha}$, e invariante por isomorfismos canônicos. Por abuso de notação, deixamos o termo medida transversal referir-se também à cada medida $v_{\alpha}$ em $\Sigma_{\alpha}$. Toda trajetória recorrente não-trivial pode ser provida com uma medida transversal (ver, por exemplo, [20, 39]). Reciprocamente, se $\Sigma$ é um segmento transversal (resp. círculo transversal) passando por $\gamma$, então toda medida de probabilidade de Borel não-atômica e $P$-invariante em $\Sigma$ induz uma medida transversal em uma pequena vizinhança de $\gamma$. Relembramos que uma medida é não-atômica ou não tem átomos se ela associar medida zero a todo conjunto discreto.

Sejam $N$ uma variedade diferenciável, $f: N \rightarrow \mathbb{R}$ uma função Borel mensurável definida em $\operatorname{dom}(f) \subset N$ e $v$ uma medida de Borel em $N$. Definimos:

$$
f^{+}(x)=\max \{f(x), 0\}, \quad f^{-}(x)=\max \{-f(x), 0\} .
$$

Dizemos que $f$ é $v$-quase-integrável com relação à medida $v$ se

$$
\min \left\{\int f^{+} d v, \int f^{-} d v\right\}<\infty
$$

e neste caso definimos

$$
\int f d v=\int f^{+} d v-\int f^{-} d v
$$


que é um número bem definido da reta real estendida $[-\infty, \infty]$.

Sejam $X \in \mathfrak{X}^{r}(M)$ e $p \in M$. O divergente de $X$ em $p$, denotado $\operatorname{div} X(p)$, é definido como sendo o traço da derivada de $X$ em $p$.

Introduzimos agora duas classes de campos vetoriais.

Definição 0.0.2 (A classe $\mathscr{C}$ ) Sejam $M$ uma variedade bidimensional compacta, $r \geq 2$ um inteiro e $X \in \mathfrak{X}^{r}(M)$. Dizemos que uma trajetória positivamente recorrente não-trivial $\gamma$ de $X$ tem a propriedade $(\star)$ se existirem $p \in \gamma$, um segmento transversal $\Sigma$ passando por $p, 0<\lambda<1$, e $N \in \mathbb{N}$ tal que a correspondente transformação de primeiro retorno $P: \Sigma \rightarrow \Sigma$ tem a seguinte propriedade:

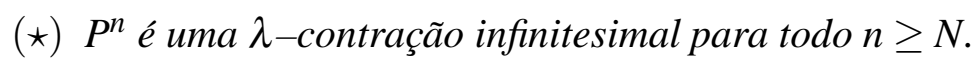

Denominamos $\lambda$ a constante de contração. Denotamos por $\mathscr{C}$ a classe de todos os campos vetoriais de classe $C^{r}$ que têm singularidades, todas hiperbólicas, e uma trajetória positivamente recorrente não-trivial satisfazendo $(\star)$.

Observação 0.0.3 Note que a Definição 0.0.2 tem um sentido local. Mais precisamente, se $\gamma$ tem a propriedade $(\star)$ e $\varepsilon>0$ é dado, então existe uma vizinhança aberta $V$ de $\gamma$ tal que cada segmento transversal $\Sigma^{\prime} \subset V$ intersectando $\gamma$ tem a propriedade $(\star)$ com constante de contração $\lambda+\varepsilon$.

Definição 0.0.4 (A classe $\mathscr{H}$ ) Sejam $M$ uma variedade bidimensional compacta, $r \geq 2$ um inteiro, e $X \in \mathfrak{X}^{r}(M)$. Dizemos que uma trajetória positivamente recorrente não-trivial $\gamma$ de $X$ tem as propriedades $(a)-(c)$ se existirem $p \in \gamma$ e um segmento transversal $\Sigma$ passando por $p$ tal que a correspondente transformação de primeiro retorno $P: \Sigma \rightarrow \Sigma$ satisfaz o seguinte:

(a) $P: \Sigma \rightarrow \Sigma$ é unicamente ergódica;

(b) A função $\log |D P|$ é $v$-quase-integrável, e $\int \log |D P| \mathrm{d} v<-c$, para algum $c>0$;

(c) O divergente de X em cada uma de suas selas é menor ou igual a zero,

onde a medida transversal $v$ em $\Sigma$ é a única medida de probabilidade em $\Sigma$ invariante por $P$. Denotamos por $\mathscr{H}$ a classe de todos os campos vetoriais de classe $C^{r}$ que têm singularidades, todas hiperbólicas, e uma trajetória positivamente recorrente não-trivial satisfazendo $(a)-(c)$. 
Observação 0.0.5 Todas as considerações feitas na Observação 0.0.3, com as devidas adaptações, podem ser aplicadas à Definição 0.0.4.

Agora enunciamos os resultados obtidos com relação ao primeira tema de pesquisa.

Teorema A. Sejam $M$ uma variedade bidimensional compacta, $r \geq 2$ um inteiro, $X \in \mathscr{C}, \gamma$ uma trajetória de $X$ satisfazendo $(\star)$, e $p \in \gamma$. Existe $Y \in \mathfrak{X}^{r}(M)$ arbitrariamente próximo de $X$ na topologia $C^{r}$ tendo uma trajetória periódica passando por $p$.

Teorema B. Sejam $M$ uma variedade bidimensional compacta, $r \geq 2$ um inteiro, e $X \in \mathscr{C}$. Existe $Y \in \mathfrak{X}^{r}(M)$ arbitrariamente próximo de $X$ na topologia $C^{r}$ tendo uma conexão de sela a mais que $X$.

Teorema C. Considere as classes de campos vetoriais $\mathscr{H}$ e $\mathscr{C}$. A seguinte inclusão vale $\mathscr{H} \subset \mathscr{C}$.

Cabe observar que para provar os Teoremas A e B foram utilizadas perturbações twist de classe $C^{r}$.

Alguns comentários históricos sobre resultados do tipo "Closing Lema de classe $C$ " são necessários para situar este trabalho dentro da pesquisa matemática em sistemas dinâmicos.

A importância do Closing Lema de classe $C^{r}$ situa-se no fato de que uma resposta positiva a ele levaria a conclusões profundas em Sistemas Dinâmicos, em tópicos relacionados à teoria de Generecidade, Estabilidade e Bifurcação. Como consequência de seu papel, há vários tipos de Closing Lema de classe $C^{r}$. Como amostra de alguns resultados importantes, gostaríamos de mencionar: o Connecting Lema de classe $C^{r}$ de Peixoto [51], o Closing Lema de classe $C^{1}$ de Pugh [54], o Closing Lemma Ergódico de classe $C^{1}$ de Mañé [41], o contraexemplo de classe $C^{r}$ do Gutierrez [22], o Closing Lema de classe $C^{r}$ do Herman [36, 37], e o Connecing Lema de classe $C^{1}$ do Hayashi [33-35]. Além dos artigos que serão citados no decorrer do texto, da mesma maneira que acima, gostaríamos de mencionar [6, 41, 48, 49, 55-58]. Como amostra de alguns resultados muito recentes que usam o Connecting Lema de classe $C^{1}$, poderíamos citar [1]. Os problemas abordados aqui estão intimamente relacionados como o seguinte problema aberto ( $\Sigma^{r}$ denota o subconjunto de $\mathfrak{X}^{r}(M)$ formado pelos campos vetoriais de Morse-Smale ):

Problema 4 (Conjectura de Peixoto) Seja M uma variedade bidimensional compacta. Um campo vetorial $X \in \mathfrak{X}^{r}(M)$ de classe $C^{r}$ é estruturalmente estável se e somente se $X \in \Sigma^{r}$. Além disso, $\Sigma^{r}$ é aberto e denso em $\mathfrak{X}^{r}(M)$. 
Peixoto [51] deu uma resposta positiva ao Problema 4 para $M$ orientável. Como conseqüência do trabalho de Peixoto e do Closing Lema de classe $C^{1}$ de Pugh $[54,58]$, segue-se que $\Sigma^{1}$ é denso em $\mathfrak{X}^{1}(M)$. Há alguns resultados parciais com relação à Conjectura de Peixoto. Em classe $C^{r}$, $r \geq 1$ : o Problem 4 é verdadeiro tanto para o plano projetivo $\mathbb{P}^{2}$ como para garrafa de Klein $\mathbb{K}^{2}$ (ver a prova em [42] de que fluxos em $\mathbb{K}^{2}$ não têm recorrência não-trivial). Gutierrez [19] simplificou a prova para $\mathbb{K}^{2}$ e mostrou que no toro com um cross-cap $\mathbb{L}^{2}$ (uma variedade não-orientável), as recorrências não-triviais são "orientáveis". Assim a prova de Peixoto para variedades bidimensionais orientáveis também funciona neste caso. Portanto, tem-se a densidade dos campos de Morse-Smale em $\mathfrak{X}^{\mathfrak{r}}(M)$ para qualquer $r \geq 1$ quando $M$ é uma variedade bidimensional orientável, ou $M=\mathbb{P}^{2}, \mathbb{K}^{2}$ ou $\mathbb{L}^{2}$. Em [51], foi mostrado que o Problema 4 é equivalente ao Connecting Lema de classe $C^{r}$ fraco (Problema 3).

Deve-se observar que as respostas parciais ao Problema 3 (e equivalentemente ao Problema 4) devidas a Peixoto e a Gutierrez, ambas utilizaram perturbações twist de classe $C^{r}$. Além disso, a resposta positiva ao Closing Lema de classe $C^{r}$, dada em [24], para uma larga classe de fluxos em variedades bidimensionais orientáveis, foi obtida por meio de perturbações twist de classe $C^{r}$ (ver também [4, 7]). Há algum tipo de obstrução para provar, em toda a sua generalidade, os problemas abordados até aqui, através de perturbações twist de classe $C^{r}$ arbitrariamente pequenas no sentido de que esta abordagem não pode abranger todos os casos [25]. Mais especificamente, em [25] mostramos que existe um campo vetorial em alguma variedade bidimensional não-orientável cujo comportamento recorrente não-trivial persiste sob perturbações twist de classe $C^{r}$ arbitrariamente pequenas do campo vetorial original.

O conteúdo até aqui apresentado integra o primeiro capítulo desta tese, intitulado “Connecting Lema e Closing Lema”. Passemos agora à segunda parte, que integra o Capítulo intitulado “Estabilidade Assintótica no Infinito para Campos Vetoriais Diferenciáveis".

Ao estudarem campos vetoriais planares de classe $C^{1}$, Markus e Yamabe conjecturaram o seguinte:

Problema 5 (Conjectura de Markus-Yamabe) Seja X um campo vetorial de classe $C^{1}$ em $\mathbb{R}^{n}$ tal que $X(0)=0$. Se, em todo o $\mathbb{R}^{n}$, as partes reais dos autovalores da derivada de $X$ são estritamente menores do que zero, então a origem é um atrator global.

Esta conjectura foi resolvida por Gutierrez [23] no caso de campos vetoriais planares de classe $C^{1}$. Uma versão deste resultado para campos apenas diferenciáveis, não necessariamente de classe $C^{1}$, foi conseguida mais recentemente por Gutierrez et al [15]. Sabe-se que este problema pode ser 
reduzido ao estudo da injetividade de aplicações planares [16].

O trabalho pioneiro de C. Olech $[46,47]$ mostrou a existência de uma forte conexão entre a estabilidade assintótica global de um campo vetorial $X: \mathbb{R}^{2} \rightarrow \mathbb{R}^{2}$ e a injetividade de $X$ (considerado como uma aplicação). Esta conexão foi fortalecida e ampliada em trabalhos subseqüentes (ver por exemplo [11, 15-18, 23, 26-30]). Este trabalho dá prosseguimento a este estudo.

Para entender o comportamento global de um campo vetorial planar é absolutamente necessário entender seu comportamento em torno do infinito. Neste sentido, o infinito pode ser considerado como uma singularidade de um campo vetorial $X: \mathbb{R}^{2} \rightarrow \mathbb{R}^{2}$. Tem havido um grande interesse no estudo local de campos vetoriais em torno de suas singularidades. Uma amostragem deste estudo são os trabalhos feitos por C. Chicone, F. Dumortier, J. Sotomayor, R. Roussarie, F. Takens (ver por exemplo $[9,13,14,59,62])$.

O problema que consideramos foi abordado no caso $C^{1}$ por Gutierrez e Teixeira [28]. Estendemos este resultado para o caso em que o campo vetorial é apenas diferenciável, não necessariamente de classe $C^{1}$.

No próximo teorema, $\sigma>0, \bar{D}_{\sigma}=\left\{z \in \mathbb{R}^{2}:\|z\| \leq \sigma\right\}$, e $\operatorname{Spec}(X)$ denota o conjunto dos autovalores de $D_{p} X$ quando $p$ percorre o conjunto $\bar{D}_{\sigma}$. Como usualmente, $\mathscr{R}(z)$ denote a parte real do número complexo $z$ e Traço $(D X): U \rightarrow \mathbb{R}$ refere-se à função que a cada ponto $p \in U$ assume o valor $\operatorname{Traço}\left(D_{p} X\right)$.

Agora seja

$\mathscr{D}(U)=\left\{X: U \rightarrow \mathbb{R}^{2}: X\right.$ é diferenciável e Traço $(D X)$ é Lebesgue quase-integrável em $\left.U\right\}$.

Definimos o índice de $X \in \mathscr{D}\left(\mathbb{R}^{2} \backslash \bar{D}_{\sigma}\right)$ no infinito como sendo o número da reta real estendida $[-\infty, \infty]$ definido por

$$
\mathscr{I}(X)=\int_{\mathbb{R}^{2}} \operatorname{Traço}(D \widehat{X}) d x \wedge d y
$$

onde $\widehat{X} \in \mathscr{D}\left(\mathbb{R}^{2}\right)$ é qualquer extensão globalmente injetiva diferenciável de $\left.X\right|_{\mathbb{R}^{2} \backslash D_{s}}$, para algum $s>\sigma$, o divergente da qual é Lebesgue quase-integrável em $\mathbb{R}^{2}$. Mostraremos (ver Corolário 2.2.12) que $\mathscr{I}(X)$ está bem definido. Podemos enunciar agora o seguinte resultado.

Teorema D. Seja $X: \mathbb{R}^{2} \backslash \bar{D}_{\sigma} \rightarrow \mathbb{R}^{2}$ um campo vetorial diferenciável (mas não necessariamente de classe $\left.C^{1}\right)$. Se para algum $\varepsilon>0$, $\operatorname{Spec}(X)$ é disjunto de $(-\varepsilon, 0] \cup\{z \in \mathbb{C}: \mathscr{R}(z) \geq 0\}$, então 
a) Para todo $p \in \mathbb{R}^{2} \backslash \bar{D}_{\sigma}$, existe uma única semitrajetória positiva de X se iniciando em $p$;

b) $\mathscr{I}(X)$, o índice de $X$ no infinito, é um número bem definido da reta real estendida $[-\infty, \infty)$;

c) Existe um vetor constante $v \in \mathbb{R}^{2}$ tal que se $\mathscr{I}(X)$ é $<0$ (resp. $\geq 0$ ), então o ponto no infinito $\infty$ da esfera de Riemann $\mathbb{R}^{2} \cup\{\infty\}$ é um repulsor (resp. um atrator) do campo vetorial $X+v$. 


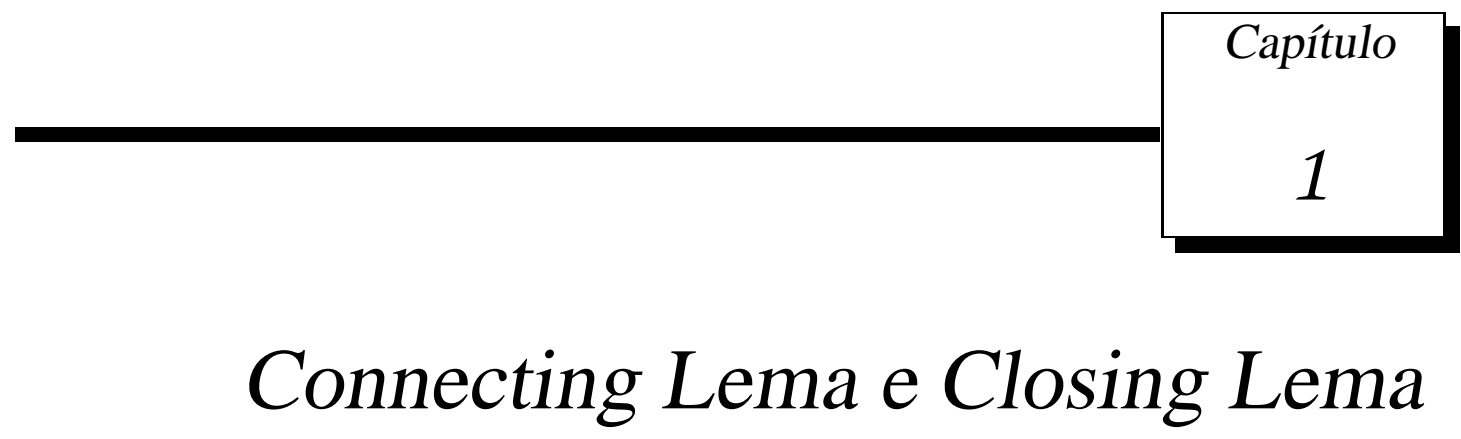

\subsection{Introdução}

Neste capítulo provaremos os Teoremas A, B, e C enunciados na introdução. É conveniente relembrar as definições abaixo.

Definição 1.1.1 (A classe $\mathscr{C}$ ) Sejam $M$ uma variedade bidimensional compacta, $r \geq 2$ um inteiro e $X \in \mathfrak{X}^{r}(M)$. Dizemos que uma trajetória positivamente recorrente não-trivial $\gamma$ de $X$ tem a propriedade $(\star)$ se existirem $p \in \gamma$, um segmento transversal $\Sigma$ passando por $p, 0<\lambda<1$, e $N \in \mathbb{N}$ tal que a correspondente transformação de primeiro retorno $P: \Sigma \rightarrow \Sigma$ tem a seguinte propriedade:

(*) $P^{n}$ é uma $\lambda$-contração infinitesimal para todo $n \geq N$.

Denominamos $\lambda$ a constante de contração. Denotamos por $\mathscr{C}$ a classe de todos os campos vetoriais de classe $C^{r}$ que têm singularidades, todas hiperbólicas, e uma trajetória positivamente recorrente não-trivial satisfazendo $(\star)$.

Definição 1.1.2 (A classe $\mathscr{H}$ ) Sejam $M$ uma variedade bidimensional compacta, $r \geq 2$ um inteiro, e $X \in \mathfrak{X}^{r}(M)$. Dizemos que uma trajetória positivamente recorrente não-trivial $\gamma$ de $X$ tem as propriedades $(a)-(c)$ se existir $p \in \gamma$ e um segmento transversal $\Sigma$ passando por $p$ tal que a correspondente transformação $P: \Sigma \rightarrow \Sigma$ de primeiro retorno satisfaz o seguinte:

(a) $P: \Sigma \rightarrow \Sigma$ é unicamente ergódica; 
(b) A função $\log |D P|$ é $v$-quase-integrável, e $\int \log |D P| \mathrm{d} v<-c$, para algum c $>0$;

(c) O divergente de X em cada uma de suas selas é menor ou igual a zero,

onde a medida transversal $v$ em $\Sigma$ é a única medida de probabilidade em $\Sigma$ invariante por $P$. Denotamos por $\mathscr{H}$ a classe de todos os campos vetoriais de classe $C^{r}$ que têm singularidades, todas hiperbólicas, e uma trajetória positivamente recorrente não-trivial satisfazendo $(a)-(c)$.

Os respectivos enunciados são os seguintes:

Teorema A. Sejam $M$ uma variedade bidimensional compacta, $r \geq 2$ um inteiro, $X \in \mathscr{C}, \gamma$ uma trajetória de $X$ satisfazendo $(\star)$, e $p \in \gamma$. Existe $Y \in \mathfrak{X}^{r}(M)$ arbitrariamente próximo de $X$ na topologia $C^{r}$ tendo uma trajetória periódica passando por $p$.

Teorema B. Sejam $M$ uma variedade bidimensional compacta, $r \geq 2$ um inteiro, e $X \in \mathscr{C}$. Existe $Y \in \mathfrak{X}^{r}(M)$ arbitrariamente próximo de $X$ na topologia $C^{r}$ tendo uma conexão de sela a mais que $X$.

Teorema C. Considere as classes de campos vetoriais $\mathscr{H}$ e $\mathscr{C}$. A seguinte inclusão vale $\mathscr{H} \subset \mathscr{C}$.

\subsection{Preliminares}

Nesta seção introduzimos a terminologia e a notação que será usada adiante. Seja $M$ uma variedade bidimensional de classe $C^{\infty}$, compacta, conexa, sem fronteira e seja $\mathfrak{X}^{r}(M), r \geq 1$, o espaço dos campos vetoriais de classe $C^{r}$ em $M$. Assumimos que $\mathfrak{X}^{r}(M)$ é dotado com a topologia $C^{r}$. Um fluxo de classe $C^{r}$ em $M$ é uma $\mathbb{R}$-ação, isto é, uma aplicação $\phi: \mathbb{R} \times M \rightarrow M$ de classe $C^{r}$ tal que $\phi(s, \phi(t, x))=\phi(s+t, x)$. Usamos a notação $\phi_{t}(x)=\phi(t, x)$. Um campo vetorial $X \in \mathfrak{X}^{r}(M)$ de classe $C^{r}$ determina um único fluxo $\phi$ de classe $C^{r}$ em $M$. A semitrajetória positiva (resp. semitrajetória negativa) passando por $p \in M$ é o conjunto

$$
\gamma_{p}^{+}=\left\{\phi_{t}(p): t \in[0, \infty)\right\} \quad\left(\text { resp. } \gamma_{p}^{-}=\left\{\phi_{t}(p): t \in(-\infty, 0]\right\}\right)
$$

A trajetória de $X$ passando por $p$ é o conjunto $\gamma_{p}=\gamma_{p}^{+} \cup \gamma_{p}^{-}$. Um arco de trajetória de $X$ é um subconjunto conexo de uma trajetória. Um ponto $p \in M$ é uma singularidade (resp. um ponto regular) 
se $X(p)=0$ (resp. $X(p) \neq 0$ ). Um ponto $p \in M$ é um ponto periódico se $p$ não é uma singularidade e $\phi_{t}(p)=p$ para algum $t>0$. Uma trajetória de $X$ é, ou um único ponto (uma singularidade) ou uma curva imersa (uma trajetória regular). Uma trajetória regular é uma trajetória periódica se ela contiver somente pontos periódicos.

Uma subvariedade unidimensional $\Sigma$ de $M$ é dita ser uma transversal ao campo vetorial $X \in \mathfrak{X}^{r}(M)$ se $\Sigma$ não contiver nenhuma singularidade de $X$ e for transversal a cada trajetória regular de $X$ (por definição, $\Sigma$ é transversal a toda trajetória que não a intercepta). Uma transversal $\Sigma$ é um segmento transversal (resp. um círculo transversal) se ela for homeomorfa a um subintervalo de $\mathbb{R}$ (resp. homeomorfa ao círculo $\mathbb{S}^{1}$ ). Dado um segmento transversal $\Sigma$, denotamos por $\partial \Sigma$ o conjunto dos extremos de $\Sigma$ e definimos $\Sigma^{\circ}=\Sigma \backslash \partial \Sigma$. Assumimos que todos os segmentos transversais são fechados $(\Sigma \supset \partial \Sigma)$ e não-degenerados $(\stackrel{\circ}{\Sigma} \neq \emptyset)$. Dizemos que um segmento transversal $\Sigma$

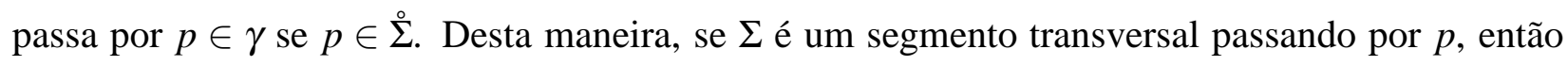
$\Sigma \backslash\{p\}$ tem duas componentes conexas (resp. uma componente conexa).

Sejam $X \in \mathfrak{X}^{\mathfrak{r}}(M)$ e $V$ uma subvariedade de $M$ disjunta das singularidades de $X$. Dizemos que $V$ é uma caixa de fluxo de $X$ do tipo retangular (resp. tipo cilíndrica) se existir um difeomorfismo $H:[0, T] \times[-1,1] \rightarrow V$ (resp. $H:[0, T] \times \mathbb{R} / \mathbb{Z} \rightarrow V)$ de classe $C^{r}$ tal que $H(t, y)=\phi_{t}(H(0, y))$, para todo $t \in[0, T]$ e $y \in[-1,1]$ (resp. $y \in \mathbb{R} / \mathbb{Z}$ ), onde $T>0$ e $\phi$ é o fluxo associado com $X$. O difeomorfismo inverso $g=H^{-1}$ é denominado um difeomorfismo retificador para $V$. $\mathrm{O}$ segmento transversal $H(\{0\} \times[-1,1])$ (resp. círculo transversal $H(\{0\} \times \mathbb{R} / \mathbb{Z})$ ) é denominado a lateral esquerda de $V$. Similarmente, definimos a lateral direita de $V$ como sendo o segmento transversal $H(\{T\} \times[-1,1])$ (resp. o círculo transversal $H(\{T\} \times \mathbb{R} / \mathbb{Z})$ ). Dada uma transversal $\Sigma$ a $X$ (i.e. um segmento transversal ou um círculo transversal) e uma caixa de fluxo $V$ de $X$, dizemos que $V$ é uma caixa de fluxo especial para $\Sigma$ se a lateral esquerda de $V$ for precisamente $\Sigma$. Com respeito à existência de caixas de fluxo, apresentamos agora uma versão do Teorema da Caixa de Fluxo tal qual enunciado por Hirsch em [38].

Teorema 1.2.1 (Teorema da Caixa de Fluxo) Sejam $X \in \mathfrak{X}^{r}(M)$, x um ponto regular não-periódico de $X$ e $\Sigma$ uma transversal a $X$. As seguintes afirmações são verdadeiras:

(a) Para cada $T>0$, existe uma caixa de fluxo $V$, do tipo retangular, contendo $x$, e um difeomorfismo retificador $g: V \rightarrow[0, T] \times[-1,1]$ tal que $g(p)=(0,0)$;

(b) Se $\Sigma$ é um segmento transversal a X então existe uma caixa de fluxo especial $V$ para $\Sigma$ do tipo retangular; Mais especificamente, existem $T>0$, uma caixa de fluxo $V$ contendo $\Sigma$, $e$ um difeomorfismo retificador $g: V \rightarrow[0, T] \times[-1,1]$ tal que $g(\Sigma)=\{0\} \times[-1,1]$; 
(c) Se $\Sigma$ é um círculo transversal a X então existe uma caixa de fluxo especial $V$ para $\Sigma$ do tipo cilíndrica; Mais especificamente, existem $T>0$, uma caixa de fluxo $V$ contendo $\Sigma$, e um difeomorfismo retificador $g: V \rightarrow[0, T] \times \mathbb{R} / \mathbb{Z}$ tal que $g(\Sigma)=\{0\} \times \mathbb{R} / \mathbb{Z}$.

Demonstração: Ver Hirsch [38, Teorema 2].

Observamos que o Teorema da Caixa de Fluxo é muito útil para realizar construções geométricas com fluxos (ver, por exemplo, Hartman [32] ou Palis e de Melo [50]).

Seja $\gamma_{p}$ uma trajetória de $X \in \mathfrak{X}^{r}(M)$. Denotamos o conjunto $\omega$-limite (resp. conjunto $\alpha$-limite) de $\gamma_{p}$ por $\omega\left(\gamma_{p}^{+}\right)$ou $\omega\left(\gamma_{p}\right)$ (resp. $\alpha\left(\gamma_{p}^{-}\right)$ou $\alpha\left(\gamma_{p}\right)$ ). A trajetória $\gamma_{p}$ é dita ser positivamente recorrente (resp. negativamente recorrente) se $\gamma_{p} \subset \omega\left(\gamma_{p}\right)$ (resp. $\gamma_{p} \subset \alpha\left(\gamma_{p}\right)$ ). Uma trajetória que é positivamente recorrente ou negativamente recorrente (resp. positivamente recorrente e negativamente recorrente) é dita ser uma trajetória recorrente (resp. trajetória recorrente bilateral). Uma trajetória recorrente pode ser trivial (uma singularidade ou uma trajetória periódica) ou não-trivial. Dizemos que um ponto $p \in M$ é recorrente (resp. positivamente recorrente, recorrente não-trivial, etc...) conforme $\gamma_{p}$ seja recorrente (resp. positivamente recorrente, recorrente não-trivial, etc...)

Seja $p \in M$ uma singularidade de $X \in \mathfrak{X}^{r}(M)$. Denotamos por $\operatorname{Spec}\left(D X_{p}\right)$ o conjunto de autovalores da derivada de $X$ em $p$. De acordo com isto, $\operatorname{Spec}\left(D X_{p}\right) \subset \mathbb{C}$. Dizemos que $p$ é uma singularidade hiperbólica se $\operatorname{Spec}\left(D X_{p}\right)$ é disjunto do eixo imaginário. Com relação a seu comportamento assintótico, uma singularidade hiperbólica pode ser de três tipos: atratora, repulsora ou uma sela.

Sejam $p, q \in M$ selas hiperbólicas de $X \in \mathfrak{X}^{r}(M)$. Dizemos que uma trajetória $\gamma$ de $X$ é uma separatriz estável de $p$ (resp. separatriz instável de $p$ ), se $\omega(\gamma)=p(\operatorname{resp} . \alpha(\gamma)=p$ ). No caso em que $\alpha(\gamma)=p$ e $\omega(\gamma)=q$ dizemos que $\gamma$ é uma conexão de selas. Se, além disso, $p=q$, então $\gamma$ é denominado um loop. Um grafo de $X$ é um subconjunto fechado e conexo de $M$ consistindo de selas $\left\{p_{i}\right\}_{i=1}^{m}$ e conexões de sela $\left\{\gamma_{i}\right\}_{i=1}^{m}$ tais que $\alpha\left(\gamma_{i}\right)=p_{i}$, para todo $1 \leq i \leq m$, e $\omega\left(\gamma_{i}\right)=p_{i+1}$ para todo $1 \leq i \leq m-1$, e $\omega\left(\gamma_{m}\right)=p_{1}$. Quando $m=1$, o grafo é um loop.

Seja $\Sigma_{1}$ (resp. $\Sigma_{2}$ ) uma transversal a $X \in \mathfrak{X}^{r}(M)$. A transformação de Poincaré induzida pelo fluxo $\phi$ associado com $X$ é a aplicação $P: \Sigma_{1} \rightarrow \Sigma_{2}$, não necessariamente definida em todos os pontos, tal que $p \in \operatorname{dom}(P)$ e $P(p)=q$ se e somente se $p \in \Sigma_{1}, \phi(\tau, p)=q \in \Sigma_{2}$ para algum $\tau>0$ e $\left\{\phi_{t}(p) \mid 0<t<\tau\right\}$ é disjunto de $\Sigma_{1} \cup \Sigma_{2}$. Quando $\Sigma_{1}=\Sigma_{2}=\Sigma$, denominamos $P: \Sigma \rightarrow \Sigma$ a transformação de primeiro retorno. Sempre assumiremos $\operatorname{dom}(P) \neq \emptyset$.

Com relação a transformações de primeiro retorno induzidas em círculos transversais, temos o 
seguinte resultado.

Teorema 1.2.2 Seja $X \in \mathfrak{X}^{r}(M)$ um campo vetorial de classe $C^{r}$ que tem singularidades, todas hiperbólicas, e uma trajetória recorrente não-trivial $\gamma$. Sejam $C$ um círculo transversal a X passando por $\gamma$ e $P: C \rightarrow C$ a correspondente transformação de primeiro retorno. Então o domínio de $P, \operatorname{dom}(P)$, é um subconjunto próprio de $C$ constituído por um número finito de intervalos abertos dois a dois disjuntos:

$$
\operatorname{dom}(P)=\bigcup_{n=1}^{r}\left(a_{n}, b_{n}\right) .
$$

Além disso, para cada $1 \leq n \leq r, \gamma_{a_{n}}^{+}$e $\gamma_{b_{n}}^{+}$vão para selas hiperbólicas de $X$. Ainda, $\left.P\right|_{\left(a_{n}, b_{n}\right)} e^{\prime}$ um difeomorfismo de classe $C^{1}$ sobre a sua imagem e uma aplicação uniformemente contínua para cada $1 \leq n \leq r$.

Demonstração: Ver Palis e de Melo [50, p. 145].

Observação 1.2.3 As conclusões do Teorema 1.2.2 também valem para a aplicação inversa $P^{-1}: C \rightarrow C$ que, de fato, é uma transformação de primeiro retorno do campo vetorial $-X$.

\subsection{Fluxos em Superfícies}

Seja $X \in \mathfrak{X}^{r}(M)$. Um conjunto que é o fecho de uma trajetória recorrente não-trivial de $X$ é denominado um conjunto quasiminimal de $X$. O próximo Teorema, devido à Cherry [8], esclarece a estrutura dos conjuntos quasiminimais.

Teorema 1.3.1 (Cherry) Todo conjunto quasiminimal de um campo vetorial $X \in \mathfrak{X}^{r}(M)$ contém um contínuo de trajetórias recorrentes bilaterais não-triviais, cada uma das quais é densa no conjunto quasiminimal.

Demonstração: Para uma prova, ver [3, Teorema 2.1, p. 57] ou [45, Teorema 2.3.3, p. 21].

Proposição 1.3.2 Seja $X \in \mathfrak{X}^{r}(M)$ um campo vetorial de classe $C^{r}$ que tem uma trajetória recorrente não-trivial $\gamma$ passando através de um segmento transversal (resp. círculo transversal) $\Sigma$ $a X$. Então $X$ também tem uma trajetória recorrente bilateral não-trivial $\widetilde{\gamma}, \operatorname{com} \operatorname{cl}(\gamma)=\operatorname{cl}(\widetilde{\gamma})$, passando por $\Sigma$ e satisfazendo $\operatorname{cl}(\gamma \cap \Sigma)=\operatorname{cl}(\widetilde{\gamma} \cap \Sigma)$. 
Demonstração: É uma consequência direta do Teorema 1.3.1.

Desta maneira, não há perda de generalidade em sempre assumir que uma trajetória recorrente não-trivial é também um trajetória recorrente bilateral não-trivial.

Apresentamos a seguir alguns teoremas de fluxos em superfícies que serão muito úteis.

Teorema 1.3.3 (Teorema de Estrutura dos Conjuntos Quasiminimais) Seja $X \in \mathfrak{X}^{r}(M)$ um campo vetorial de classe $C^{r}$ que tem número finito de singularidades, todas hiperbólicas, e seja $N$ um conjunto quasiminimal de $X$. Então toda trajetória recorrente não-trivial de $\left.X\right|_{N}$ é densa em $N$. Além disso, $N$ contém somente os seguintes tipos de trajetórias:

(i) Trajetórias recorrentes bilaterais não-triviais ;

(ii) Separatrizes que são trajetórias recorrentes não-triviais;

(iii) Separatrizes conectando selas hiperbólicas (loops são permitidos);

(iv) Selas Hiperbólicas.

Demonstração: Ver [45, p. 31].

Teorema 1.3.4 (Teorema de Estrutura dos Conjuntos Limites) Seja $X \in \mathfrak{X}^{r}(M)$ um campo vetorial de classe $C^{r}$ que tem um número finito de singularidades, todas hiperbólicas, e seja $\gamma_{p}^{+}$(resp. $\left.\gamma_{p}^{-}\right)$uma semitrajetória positiva (resp. negativa) de X. Então $\omega\left(\gamma_{p}^{+}\right)\left(\right.$resp. $\left.\alpha\left(\gamma_{p}^{-}\right)\right)$é um e somente um dos seguintes conjuntos compactos não-vazios:

(i) Uma singularidade

(ii) Uma trajetória periódica;

(iii) Um grafo;

(iv) Um conjunto quasiminimal.

Demonstração: Ver [45, pp. 35-36].

Sejam $X \in \mathfrak{X}^{r}(M)$ e $\phi$ o fluxo associado com $X$. Dizemos que $N \subset M$ é um conjunto invariante de $X$ (ou um conjunto $\phi$-invariante) se $\phi_{t}(N) \subset N$, para todo $t \in \mathbb{R}$. Dizemos que $K \subset M$ é um conjunto minimal de $X$ se $K$ é fechado, não-vazio e invariante, e não existe nenhum subconjunto próprio de $K$ com estas propriedades. Os conjuntos minimais de um campo vetorial de classe $C^{2}$ foram classificados por Denjoy-Schwartz. 
Teorema 1.3.5 (Denjoy-Schwartz) Sejam $X \in \mathfrak{X}^{r}(M), r \geq 2$, um campo vetorial de classe $C^{r}$ e $S$ um conjunto minimal de X. Então exatamente um dos seguintes casos ocorre:

(i) S consiste de uma singularidade;

(ii) S é uma trajetória periódica;

(iii) $S=M$, e neste caso $M$ é o toro $\mathbb{T}^{2}$ e X é topologicamente equivalente ao fluxo irracional.

Demonstração: Sob as hipóteses sobre $X$, é fácil ver que $S$ é uma singularidade, uma trajetória periódica, ou $S=M$ (ver [60] ou [12, pp. 39-40]). Neste último caso, por um resultado de Markley [43], $M=\mathbb{T}^{2}$, e por um resultado de Gutierrez [21], $X$ é topologicamente equivalente ao fluxo irracional.

Com relação a um campo vetorial $X \in \mathfrak{X}^{1}(M)$ de classe $C^{1}$, sabe-se que se $\gamma$ é uma trajetória recorrente não-trivial de $X$ então existem uma separatriz instável $\gamma_{1}$ e uma separatriz estável $\gamma_{2}$ tais que $\omega\left(\gamma_{1}\right) \cap \alpha\left(\gamma_{2}\right) \supset \gamma$ (ver [50]). Se, além disso, o campo é de classe $C^{2}$, então estas separatrizes podem ser tomadas dentro do mesmo conjunto quasiminimal que contém $\gamma$. Isto é o que mostra o próximo resultado.

Proposição 1.3.6 Seja $X \in \mathfrak{X}^{r}(M), r \geq 2$, um campo vetorial de classe $C^{r}$ que tem singularidades, todas hiperbólicas, e um trajetória recorrente não-trivial $\gamma$. Então existem uma separatriz de sela $\gamma_{1}$ instável e uma separatriz de sela $\gamma_{2}$ estável, ambas trajetórias recorrentes não-triviais de $X$ satisfazendo $\mathrm{cl}\left(\gamma_{1}\right)=\mathrm{cl}\left(\gamma_{2}\right)=\mathrm{cl}(\gamma)$, tais que para qualquer círculo transversal (resp. segmento transversal) $\Sigma$ a X passando por $\gamma$ temos que

(a) $\omega\left(\gamma_{1}\right) \supset \gamma_{2}$ e $\alpha\left(\gamma_{2}\right) \supset \gamma_{1}$;

(b) $\operatorname{cl}\left(\gamma_{1} \cap \Sigma\right)=\operatorname{cl}\left(\gamma_{2} \cap \Sigma\right)=\operatorname{cl}(\gamma \cap \Sigma)$.

Demonstração: Pela Proposição 1.3.2, podemos assumir que $\gamma$ é uma trajetória recorrente nãotrivial bilateral. Primeiramente mostraremos que $\mathrm{cl}(\gamma)$ contém singularidades. Assuma o contrário. Então cl $(\gamma)$ não contém nem singularidades nem separatrizes de sela. Sob estas circunstâncias, pelo Teorema 1.3.3, temos que $\mathrm{cl}(\gamma)$ é um conjunto minimal. Agora pelo Teorema 1.3.5, $\mathrm{cl}(\gamma)=M$. Isto é uma contradição porque $M$ contém pelo menos uma singularidade. Assim, $\mathrm{cl}(\gamma)$ contém singularidades, todas sendo selas hiperbólicas. Se $p_{1} \in \operatorname{cl}(\gamma)$ é uma sela hiperbólica, como cada 
sela hiperbólica em $\mathrm{cl}(\gamma)$ é acumulada por $\gamma$, temos que $\mathrm{cl}(\gamma)$ contém pelo menos uma separatriz instável $\sigma_{1}$ de $p_{1}$ e uma separatriz estável $\tau_{1}$ de $p_{1}$. Segue-se do Teorema 1.3.3 que ou $\sigma_{1}$ é uma trajetória recorrente não-trivial que é densa em $\operatorname{cl}(\gamma)$ ou $\omega\left(\sigma_{1}\right)$ é uma sela hiperbólica $p_{2}$ contida em $\mathrm{cl}(\gamma)$. Então podemos tomar uma separatriz instável $\sigma_{2}$ de $p_{2}$ contida em $\operatorname{cl}(\gamma)$ e repetir o raciocínio. Como há um número finito de separatrizes, depois de um número finito de passos, digamos $n$, vamos obter ou uma separatriz instável positivamente recorrente $\sigma_{n}$ contida e densa em $\operatorname{cl}(\gamma)$, ou um grafo. Mas $\mathrm{cl}(\gamma)=\omega\left(\gamma^{+}\right)$de modo que pelo Teorema 1.3.4 não há grafo em $\operatorname{cl}(\gamma)$. Desta maneira, $\gamma_{1}=\sigma_{n}$ é a trajetória positivamente recorrente que procurávamos. Procedendo da mesma maneira com respeito à $\tau_{1}$, obtemos uma separatriz estável $\gamma_{2}=\tau_{m}$ em $\operatorname{cl}(\gamma)$ que é uma trajetória negativamente recorrente satisfazendo $\mathrm{cl}\left(\gamma_{2}\right)=\mathrm{cl}(\gamma)$. Isto prova $(a)$. Usando que $\operatorname{cl}\left(\gamma_{1}\right)=\operatorname{cl}\left(\gamma_{2}\right)=\operatorname{cl}(\gamma)$ e o Teorema da Caixa de Fluxo, podemos facilmente provar $(b)$.

Para adentrar a estrutura dos conjuntos quasiminimais, é necessário usar o Teorema de Estrutura do Gutierrez [21]. Para tanto, faz-se necessário introduzir algumas definições. Uma transformação de intercâmbio de intervalos, abreviadamente iet, é uma aplicação diferenciável injetora $T: \mathbb{R} / \mathbb{Z} \rightarrow \mathbb{R} / \mathbb{Z}$ definida em $\mathbb{R} / \mathbb{Z}$ menos finitos pontos e tal que $\left|T^{\prime}(x)\right|=1$ para todo $x \in \operatorname{dom}(T)$ (seu domínio de definição). A semitrajetória positiva (resp. negativa) de $T$ passando pelo ponto $x \in \mathbb{R} / \mathbb{Z}$ é o conjunto

$$
O^{+}(x)=\left\{T^{n}(x) / n \in \mathbb{Z}, n \geq 0 \text { e } x \in \operatorname{dom}\left(T^{n}\right)\right\}
$$

(resp. $O^{-}(x)=\left\{T^{n}(x) / n \in \mathbb{Z}, n \leq 0\right.$ e $\left.x \in \operatorname{dom}\left(T^{n}\right)\right\}$ ), onde $T^{0}$ denota a aplicação identidade de $\mathbb{R} / \mathbb{Z}$. A trajetória de $E$ passando por $x$ é o conjunto $O(x)=O^{+}(x) \cup O^{-}(x)$. Dizemos que a trajetória $O(x)$ é infinita (resp. finita) se ela for um conjunto enumerável (resp. finito). Uma iet é minimal se toda trajetória dela é infinita e densa em $\mathbb{R} / \mathbb{Z}$. O conjunto $A \subset O(p)$ é um arco de trajetória de $T$ conectando os pontos $x$ e $y$ se existirem $m>0$ e uma ordenação $A=\left\{p_{n}\right\}_{n=0}^{m}$ de $A$, with $p_{0}=x$ e $p_{m}=y$ tal que $p_{n+1}=T\left(p_{n}\right)$ para todo $0 \leq n \leq m-1$. As definições anteriores também valem para aplicações de primeiro retorno.

Seja $f: M_{1} \rightarrow M_{1}$ (resp. $g: M_{2} \rightarrow M_{2}$ ) uma transformação de uma variedade $M_{1}$ (resp. $M_{2}$ ) nela mesma, com domínio $\operatorname{dom}\left(f_{1}\right) \subset M_{1}$ (resp. $\left.\operatorname{dom}\left(f_{2}\right) \subset M_{2}\right)$. Dizemos que $f_{1}$ é topologicamente conjugada (resp. topologicamente semiconjugada) a $f_{2}$ se existir um homeomorfismo (resp. uma aplicação contínua sobrejetora que preserva orientação) $h: M_{1} \rightarrow M_{2}$ tal que $h\left(\operatorname{dom}\left(f_{1}\right)\right) \subset$ $\operatorname{dom}\left(f_{2}\right)$ e $h \circ f_{1}(z)=f_{2} \circ h(z)$ para todo $z \in \operatorname{dom}\left(f_{1}\right)$. Neste caso, $h: M_{1} \rightarrow M_{2}$ é denominada uma conjugação (resp. semiconjugação) entre $f_{1}$ e $f_{2}$. 
Relembramos que o número de conjuntos quasiminimais de um fluxo em um variedade compacta bidimensional é finito. Mais especificamente, vamos precisar do seguinte resultado.

Teorema 1.3.7 Seja $\phi: \mathbb{R} \times M \rightarrow M$ um fluxo contínuo em uma variedade bidimensional compacta M. O fecho das trajetórias recorrentes não-triviais de $\phi$ determina um número finito de subconjuntos $N_{1}, N_{2}, \ldots, N_{m}$ de $M$ compactos e $\phi$-invariantes tais que qualquer trajetória recorrente não-trivial de $\phi$ é um subconjunto denso de algum $N_{i}$. Além disso, existe uma coleção $\left\{V_{i}\right\}_{i=1}^{m}$ de subconjuntos de $M$ abertos, conexos, dois a dois disjuntos tais que cada $V_{i}$ contém todas as trajetórias recorrentes não-triviais intersectando $N_{i}$. Cada conjunto $V_{i}$ é denominado uma região de recorrência para $N_{i}$.

\section{Demonstração: Ver [21].}

O próximo corolário é uma conseqüência imediata do Teorema 1.3.7. Como de costume, $\gamma_{x}^{+}$ denota a semitrajetória positiva de $X$ iniciando-se no ponto $x$, e $\gamma_{x}^{+}(t)$ denota a parametrização de $\gamma_{x}^{+}$induzida pelo fluxo.

Corolário 1.3.8 Seja $X \in \mathfrak{X}^{r}(M)$ um campo vetorial de classe $C^{r}$ que tem singularidades, todas hiperbólicas, e uma trajetória recorrente não-trivial $\gamma$. Existe uma vizinhança aberta $U$ de $\gamma$ tal que para qualquer segmento transversal (resp. círculo transversal) $\Sigma$ passando por $\gamma$ e contido em $U$ o seguinte vale $(P: \Sigma \rightarrow \Sigma$ denota a correspondente transformação de primeiro retorno $)$ :

(i) $\Sigma$ não intersecta nem trajetórias periódicas nem conexões de selas de X;

(ii) Para cada $x \in \cap_{n=1}^{\infty} \operatorname{dom}\left(P^{n}\right), \omega\left(\gamma_{x}^{+}\right)=\operatorname{cl}(\gamma)$, e para cada $y \in \cap_{n=1}^{\infty} \operatorname{dom}\left(P^{-n}\right), \alpha\left(\gamma_{y}^{-}\right)=$ $\operatorname{cl}(\gamma)$.

Demonstração: Sejam $\left\{N_{i}\right\}_{i=1}^{m}$ os conjuntos quasiminimais de $X$ e $\left\{V_{i}\right\}_{i=1}^{m}$ uma coleção de regiões de recorrência para $X$ como no Teorema 1.3.7. Assim $N_{i} \subset \operatorname{cl}\left(V_{i}\right)$ para cada $1 \leq i \leq m$, e $V_{i} \cap$ $V_{j}=\emptyset$ sempre que $i \neq j$. Sem perda de generalidade podemos assumir que $N_{1}=\operatorname{cl}(\gamma)$ e $\gamma \subset V_{1}$. Seja $U \subset V_{1}$ uma vizinhança aberta de $\gamma$ disjunta de trajetórias periódicas e conexões de selas de $X$ (relembramos que uma trajetória recorrente não-trivial não pode ser acumulada por trajetórias periódicas e que há somente um número finito de conexões de selas). Sejam $\Sigma \subset U$ um segmento transversal (resp. círculo transversal) passando por $\gamma$ e $P: \Sigma \rightarrow \Sigma$ a correspondente transformação de primeiro retorno. Item $(i)$ segue da construção de $U$. Vamos provar (ii). Para tanto, seja 
$x \in \cap_{n=1}^{\infty} \operatorname{dom}\left(P^{n}\right)$. Por $(i)$ temos que $\omega\left(\gamma_{x}^{+}\right)=N_{i}$ para algum $1 \leq i \leq m$. A prova de (ii) estará terminada se mostrarmos que $i=1$. Assuma o contrário. Então $\omega\left(\gamma_{x}^{+}\right)=N_{i}$ para algum $1<i \leq m$. Como $N_{i} \subset \operatorname{cl}\left(V_{i}\right) \subset M \backslash V_{1}$ e $\Sigma \subset U \subset V_{1}$, não é difícil ver que $\omega\left(\gamma_{x}^{+}\right)=N_{i}$ implica que existe $T>0$ tal que $\gamma_{x}^{+}(t) \cap \Sigma=\emptyset$, para todo $t \geq T$. Isto contradiz a hipótese de que $x \in \cap_{n=1}^{\infty} \operatorname{dom}\left(P^{n}\right)$. Assim temos que $i=1$. A prova do outro caso é similar.

Teorema 1.3.9 (Teorema de Estrutura do Gutierrez) Seja $\phi: \mathbb{R} \times M \rightarrow M$ um fluxo contínuo em uma variedade bidimensional compacta $M$. Para cada trajetória recorrente não-trivial $\gamma$ de $\phi$, existe um círculo transversal C passando por $\gamma$ e contido em uma região de recorrência associada com $\gamma$ tal que se $P: C \rightarrow C$ é a correspondente transformação de primeiro retorno então:

(a) $\mathrm{cl}(\gamma) \cap C=C$ ou $\mathrm{cl}(\gamma) \cap C$ é um conjunto de Cantor;

(b) P é topologicamente semiconjugada a uma transformação de intercâmbio de intervalos $T: \mathbb{R} / \mathbb{Z} \rightarrow \mathbb{R} / \mathbb{Z}$ minimal;

(c) Toda trajetória de $P$ é infinita. Equivalentemente, para cada $q \in C, \gamma_{q}$ intersecta $C$ um número infinito de vezes, isto é, $\gamma_{q} \cap C$ é um conjunto infinito.

Denominamos o círculo C um círculo transversal especial.

Demonstração: Ver [21].

Observação 1.3.10 Note que todo círculo transversal especial tem as propriedades $(i)$ e $(i i)$ do Corolário 1.3.8.

Proposição 1.3.11 Seja $X \in \mathfrak{X}^{r}(M)$ um campo vetorial de classe $C^{r}$ que tem singularidades, todas hiperbólicas, e uma trajetória recorrente não-trivial $\gamma$. Assuma que $C$ seja um círculo transversal a $X$ passando por $\gamma$ e seja $P: C \rightarrow C$ a correspondente transformação de primeiro retorno. Para cada $p \in C \backslash \operatorname{dom}(P)$ temos que $\omega\left(\gamma_{p}^{+}\right)$ou é uma sela hiperbólica ou existe algum segmento transversal $I_{p} \subset C$ contendo p tal que $I_{p} \subset \cap_{n=1}^{\infty} \operatorname{dom}\left(P^{-n}\right)$.

Demonstração: Primeiramente assuma que $C$ seja um círculo transversal especial e denote por $P: C \rightarrow C$ a correspondente transformação de primeiro retorno. Então ou $p$ pertence à fronteira de $\operatorname{dom}(P)$ ou existe uma vizinhança $I_{p} \subset C$ de $p$ tal que $I_{p} \cap \operatorname{dom}(P)=\emptyset$. No primeiro 
caso, pelo Teorema 1.2.2, $\omega\left(\gamma_{p}^{+}\right)$é uma sela de $X$. No segundo caso, por $(c)$ do Teorema 1.3.9, $I_{p} \subset \cap_{n=1}^{\infty} \operatorname{dom}\left(P^{-n}\right)$. Por $(i i)$ do Corolário 1.3.8 isto implica que $I_{p} \subset \cap_{n=1}^{\infty} \operatorname{dom}\left(P_{I_{p}}^{-n}\right)$, onde $P_{I_{p}}$ é a transformação de primeiro retorno induzida por $X$ em $I_{p}$. É fácil ver que qualquer subintervalo de $I_{p}$ intersectando $\gamma$ herda esta propriedade. Mais geralmente, usando transformações de Poincaré, podemos mostrar que qualquer segmento transversal suficientemente pequeno passando por $\gamma$ tem essa mesma propriedade. Em particular, se $\widetilde{C}$ é um círculo transversal passando por $\gamma$, existe um subintervalo $J \subset \widetilde{C}$ tal que $J \subset \cap_{n=1}^{\infty} \operatorname{dom}\left(P_{J}^{n}\right) \subset \cap_{n=1}^{\infty} \widetilde{P}^{n}$, onde $P_{J}: J \rightarrow J$ (resp. $\widetilde{P}: \widetilde{C} \rightarrow \widetilde{C}$ ) é a transformação de primeiro retorno induzida por $X$ em $J$ (resp. em $\widetilde{C}$ ).

A próxima proposição diz que dada uma trajetória recorrente não-trivial $\gamma$ de $X \in \mathfrak{X}^{r}(M)$, podemos sempre construir círculos transversais passando por $\gamma$ que herdam as propriedades de segmentos transversais passando por $\gamma$. Vamos precisar das seguintes definições:

$$
\Omega_{+}=\bigcap_{n=1}^{\infty} \operatorname{dom}\left(P^{n}\right), \quad \Omega_{-}=\bigcap_{n=1}^{\infty} \operatorname{dom}\left(P^{-n}\right)
$$

e do seguinte lema.

Lema 1.3.12 Seja $P: \Sigma \rightarrow \Sigma$ uma transformação de primeiro retorno suave tendo a propriedade $(\star)$. Então para cada $0<\lambda<1$, existe $N \in \mathbb{N}$ tal que $P^{n}$ é uma $\lambda$-contração infinitesimal para todo $n \geq N$.

Demonstração: Por hipótese, existem $0<\xi<1$ e $L \in \mathbb{N}$ tais que $P^{n}$ é uma $\xi$-contração infinitesimal para todo $n \geq L$. Dado $0<\lambda<1$, seja $q \in \mathbb{N}$ tal que $\xi^{q+1}<\lambda$. Defina $N=(q+1) L$. Podemos colocar cada $n \geq N$ na forma $n=r+q L$, onde $r \geq L$. Assim, $P^{r}$ é uma $\xi$-contração infinitesimal e $P^{q L}$ uma $\xi^{q}$-contração infinitesimal. Portanto $P^{n}=P^{r} \circ P^{q L}$ é uma $\xi^{q+1}$-contração infinitesimal e, como tal, uma $\lambda$-contração infinitesimal, para todo $n \geq N$.

Na próxima proposição, $d: U_{p} \times U_{p} \rightarrow \mathbb{R}$ denota uma função distância em uma vizinhança $U_{p}$ de $p$.

Proposição 1.3.13 Seja $X \in \mathfrak{X}^{r}(M)$ uma campo vetorial de classe $C^{r}$ que tem singularidades, todas hiperbólicas, e uma trajetória positivamente recorrente não-trivial $\gamma$ tendo a propriedade ( $\star$. Para cada $0<\lambda<1, p \in \gamma$ e $\varepsilon>0$, existem um círculo transversal $C$ passando por $\gamma$ e $N \in \mathbb{N}$ tais que a correspondente transformação de primeiro retorno $P: C \rightarrow C$ tem as seguintes propriedades: 
(i) Toda trajetória de P é infinita;

(ii) $P^{n}$ é uma $\lambda$-contração infinitesimal para todo $n \geq N$;

(iii) $\Omega_{+}$é todo o círculo C menos um conjunto enumerável de pontos, cujas trajetórias positivas vão para selas hiperbólicas de $X$;

(iv) C intersecta $\gamma$ em $\bar{p}$, onde $d(\bar{p}, p)<\varepsilon$;

Denominamos C um círculo transversal distinguido.

Demonstração: Por hipótese, como $\gamma$ tem a propriedade $(\star)$, existe um segmento transversal $\Sigma$ passando por $p, N_{1} \in \mathbb{N}$, e $0<\xi<1$ tais que a transformação de primeiro retorno $P_{\Sigma}: \Sigma \rightarrow \Sigma$ tem a seguinte propriedade: $\left(P_{\Sigma}\right)^{n}$ é uma $\xi$-contração infinitesimal para todo $n \geq N_{1}$. Além disso, pelo Teorema 1.3.9, podemos assumir que toda trajetória de $P_{\Sigma}$ é infinita. Agora procedemos com a construção do círculo transversal. Seja $\Sigma$ munido de alguma orientação. Dizemos que um par $(x, y)$ de pontos $\gamma \cap \Sigma$ é sucessivo se $x \in \operatorname{dom}\left(P_{\Sigma}\right)$ e $P_{\Sigma}(x)=y$. Substituindo $\Sigma$ por um subsegmento se necessário (isto não afeta as asserções anteriores), podemos assumir o seguinte: existe um par sucessivo de pontos $(x, y)$, com $d(x, p)<\frac{\varepsilon}{2}$, e segmentos transversais $\Sigma_{1} \subset \Sigma, \Sigma_{3} \subset \Sigma$, com $x \in \stackrel{\circ}{\Sigma}_{1}$, $y \in \stackrel{\circ}{\Sigma}_{3}, \Sigma_{1} \cap \Sigma_{3}=\emptyset$, de tal maneira que a transformação de primeiro retorno $P_{\Sigma_{1}, \Sigma_{3}}: \Sigma_{1} \rightarrow \Sigma_{3}$ é uma aplicação sobrejetora que preserva orientação e está definida em todo ponto de $\Sigma_{1}$ (ver a prova deste fato em Katok [39, pp. 453-454]). Seja $B$ a caixa de fluxo que tem $\Sigma_{1}$ e $\Sigma_{3}$ como as laterais esquerda e direita, respectivamente (ver a Figura 1.1). Por um procedimento padrão podemos construir um segmento transversal $\Sigma_{4} \subset B$ que cruza todo arco de trajetória de $X$ contido em $B$ precisamente uma vez. Então colamos ele com um segmento transversal $\Sigma_{2}$, de uma maneira suave, para obter o desejado círculo transversal $C=\Sigma_{2} \cup \Sigma_{4}$ (ver a Figura 1.1). Denote por $\gamma_{q}$ a trajetória de $X$ iniciando-se em $q$, e por \# a cardinalidade de um conjunto. Como, para qualquer $q \in \Sigma$, $\#\left(\gamma_{q} \cap \Sigma\right)=\infty$ e $x \in \gamma \subset\left(\alpha\left(\gamma_{q}\right) \cup \omega\left(\gamma_{q}\right)\right)$ (ver $(i i)$ do Corolário 1.3.8), temos que \# $\left(\gamma_{q} \cap \Sigma_{1}\right)=\infty$ sempre que $q \in \Sigma_{2}$. Agora como $\Sigma_{1}$ é levado pelo fluxo associado com $X$ difeomorficamente sobre $\Sigma_{4}$ temos que $\#\left(\gamma_{q} \cap \Sigma_{4}\right)=\infty$ sempre que $q \in \Sigma_{2}$. Em particular, \# $\left(\gamma_{q} \cap C\right)=\infty$ sempre que $q \in \Sigma_{2}$. Usando argumentos similares podemos mostrar que $\#\left(\gamma_{q} \cap C\right)=\infty$ sempre que $q \in \Sigma_{4}$. Assim, como $C=\Sigma_{2} \cup \Sigma_{4}$, temos que toda trajetória de $P$ é infinita, onde $P: C \rightarrow C$ denota a transformação de primeiro retorno induzida por $X$ em $C$. Acabamos de provar $(i)$. Para provar $(i i)$, temos de usar que $\left(P_{\Sigma}\right)^{n}$ é uma $\xi$-contração infinitesimal para todo $n \geq N_{1}$. Não é difícil mostrar que em uma vizinhança de cada $q \in \operatorname{dom}\left(P^{n}\right), P^{n}$ coincide com uma das seguintes aplicações: $P_{\Sigma_{1}, \Sigma_{4}} \circ\left(P_{\Sigma}\right)^{m(n)} \circ P_{\Sigma_{4}, \Sigma_{1}},\left(P_{\Sigma}\right)^{m(n)} \circ P_{\Sigma_{4}, \Sigma_{1}}, P_{\Sigma_{1}, \Sigma_{4}} \circ\left(P_{\Sigma}\right)^{m(n)}$ or $\left(P_{\Sigma}\right)^{m(n)}$, onde $m(n)>n$. Como $P_{\Sigma_{1}, \Sigma_{4}}$ e $P_{\Sigma_{4}, \Sigma_{1}}$ são aplicações suaves definidas em conjuntos compactos temos que existe $N_{2} \geq N_{1}$ tal 


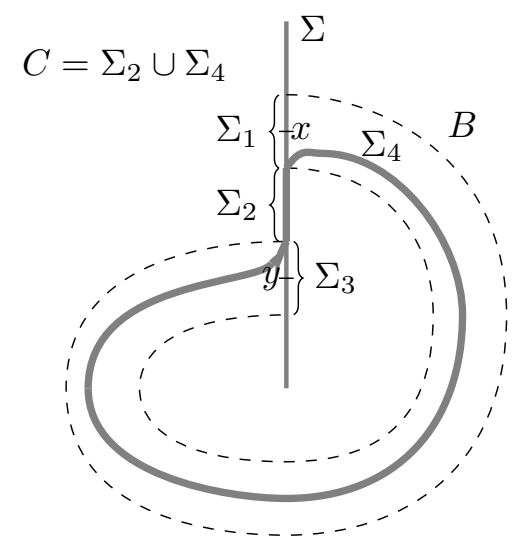

Figura 1.1: Construção do círculo transversal distinguido.

que $\left|D P^{n}(x)\right|<\xi$, para todo $n \geq N_{2}$, e $x \in \operatorname{dom}\left(P^{n}\right)$. Assim $P^{n}$ é uma $\xi$-contração infinitesimal para todo $n \geq N_{2}$. Dado $0<\lambda<1$, o Lema 1.3 .12 assegura que existe $N>N_{2}$ tal que $P^{n}$ é uma $\lambda$-contração infinitesimal para todo $n \geq N$. Isto prova (ii). Agora provamos (iii). Seja $q \in C \backslash \operatorname{dom}(P)$, e assuma que $\omega\left(\gamma_{q}^{+}\right)$não seja uma sela hiperbólica. Pela proposição 1.3.11 existe algum intervalo $I_{q} \subset C$ contendo $q$ tal que $I_{q} \subset \Omega_{-}$. Assim, $P^{-n}\left(I_{q}\right)$ está contido numa componente conexa de $\operatorname{dom}\left(P^{-n}\right)$ para todo $n \in \mathbb{N}$. Por $(i i)$, existe $N \in \mathbb{N}$ tal que $\left.P^{-n}\right|_{\Omega_{-}}$é uma $\frac{1}{\lambda}$-expansão infinitesimal para todo $n \geq N$. Para chegar a uma contradição, seja $[a, b] \subset I_{q}$, onde $\ell([a, b])=$ $b-a>0$. Seja $I_{n}=P^{-n}([a, b])$. Então

$$
\ell\left(I_{k N}\right)=\ell\left(P^{-k N}([a, b])\right)>\frac{1}{\lambda^{k}} \ell([a, b])=\frac{1}{\lambda^{k}}(b-a)
$$

onde $\ell$ denota o comprimento de arco. Assim $\ell\left(I_{k N}\right) \rightarrow \infty$ quando $k \rightarrow \infty$, o que é um absurdo porque $I_{k N} \subset C$ e $C$ é compacto. Para provar $(i v)$, note que podemos construir o círculo transversal $C$ de tal maneira que $C$ intersecta $\gamma$ em $\bar{p} \in \Sigma_{4}$ satisfazendo $d(\bar{p}, x)<\frac{\varepsilon}{2}$. Logo $d(\bar{p}, p)<\varepsilon$.

\subsection{Tranformações de Primeiro Retorno Contrativas}

Nesta seção derivamos algumas propriedades de campos vetoriais na classe $\mathscr{C}$. Apresentamos também o conceito de perturbação twist de um campo vetorial.

Definição 1.4.1 (Perturbação de um campo vetorial) Seja $X \in \mathfrak{X}^{r}(M) e V \subset M$. Dado $Y \in \mathfrak{X}^{r}(M)$, dizemos que $X+Y$ é uma perturbação de classe $C^{r}$ de $X$ localizada em $V$ se o suporte de $Y$ estiver contido em $V$. 
Definição 1.4.2 (Perturbação twist de um campo vetorial) $\operatorname{Sejam} X \in \mathfrak{X}^{r}(M)$ e $V \subset M$ uma caixa de fluxo compacta de $X$. Dado $Y \in \mathfrak{X}^{r}(M)$ com o suporte contido em $V$, dizemos que $X+Y$ é uma perturbação twist de $X$ de classe $C^{r}$ localizada em $V$ se $\left.X\right|_{V}$ for transversal a $\left.Y\right|_{V^{\circ}}$, onde $\stackrel{\circ}{V}$ denota $o$ interior de $V$.

Definição 1.4.3 (Perturbação de uma transformação de primeiro retorno) Seja $C$ um círculo transversal a $X_{i} \in \mathfrak{X}^{r}(M), i=1,2$, e seja $P_{i}: C \rightarrow C$ a transformação de primeiro retorno induzida por $X_{i}$ em $C$. Dizemos que $P_{2}$ é uma perturbação (resp. perturbação twist) de $P_{1}$ de classe $C^{r}$ localizada em um segmento transversal $\Sigma \subset C$ se $X_{2}$ for uma perturbação (resp. perturbação twist) de $X_{1}$ de classe $C^{r}$ localizada em uma caixa de fluxo especial para $\Sigma$. Neste caso, existe um difeomorfismo $G: C \rightarrow C$ (resp. um difeomorfismo $G: C \rightarrow C$ sem pontos fixos) de classe $C^{r}$ tal que $\left.G\right|_{C \backslash \Sigma}$ é a aplicação identidade, e $P_{2}=P_{1} \circ G$ sempre que ambos os lados estiverem definidos.

Doravante, a mesma notação dos intervalos da reta será usada para subintervalos (isto é, segmentos orientados) $[a, b],[a, b), \ldots$ de um segmento transversal ou círculo transversal $\Sigma$. Desta maneira, dado $x \in \stackrel{\circ}{\Sigma}$, podemos usar expressões da forma $x+\delta, \delta>0$ pequeno, para fazer referência ao único ponto de $\Sigma$ satisfazendo $\ell([x, x+\delta])=\delta$, onde $\ell$ denota comprimento de arco.

Definição 1.4.4 Seja $\Sigma$ um segmento transversal ou um círculo transvsersal a $X \in \mathfrak{X}^{r}(M)$. Dados $x \in \sum^{\circ} e \delta>0$ pequeno, defina $I_{x}(\delta)=[x-\delta, x+\delta] \subset \Sigma$ como sendo o subintervalo de $\Sigma$ de raio $\delta$ centrado em $x$. Vamos denotar por $V_{x}(\delta)$ qualquer caixa de fluxo especial para $I_{x}(\delta)$.

Proposição 1.4.5 Sejam $X \in \mathfrak{X}^{r}(M), \bar{p} \in M$ um ponto positivamente recorrente não-trivial de $X$, $\Sigma$ um segmento transversal ou um círculo transversal passando por $\bar{p}$, e $P: \Sigma \rightarrow \Sigma$ a correspondente transformação de primeiro retorno. Para cada $\bar{N} \in \mathbb{N}$, existe $\delta>0$ tal que $I_{\bar{p}}(2 \delta) \subset \operatorname{dom}\left(P^{\bar{N}-1}\right) e$

$$
P^{n}\left(I_{\bar{p}}(2 \delta)\right) \cap I_{\bar{p}}(2 \delta)=\emptyset \quad \text { para todo } \quad 1 \leq n \leq \bar{N}-1
$$

onde $I_{\bar{p}}(2 \delta) \subset \Sigma$ é o subintervalo de $\Sigma$ de raio $2 \delta$ centrado em $\bar{p}$.

Demonstração: Isto é uma consequência imediata do Teorema da Caixa de Fluxo.

Proposição 1.4.6 Sejam $X \in \mathfrak{X}^{r}(M), \bar{p} \in M$ um ponto positivamente recorrente não-trivial de $X$, $\Sigma$ um círculo transversal passando por $\bar{p}$, e $P: \Sigma \rightarrow \Sigma$ a correspondente transformação de primeiro 
retorno. Para cada $\bar{N} \in \mathbb{N}$, existe $\rho>0$, tal que para todo $0<\delta<\rho$ e para toda perturbação $\widetilde{P}$ de $P$ de classe $C^{r}$ localizada em $I_{\bar{p}}(\delta)$, temos que $I_{\bar{p}}(2 \delta) \subset \operatorname{dom}\left(\widetilde{P}^{\bar{N}-1}\right) e$

$$
\widetilde{P}^{n}\left(I_{\bar{p}}(2 \delta)\right) \cap I_{\bar{p}}(2 \delta)=\emptyset \quad \text { para todo } \quad 1 \leq n \leq \bar{N}-1
$$

onde $I_{\bar{p}}(\boldsymbol{\delta})$ (resp. $\left.I_{\bar{p}}(2 \delta)\right)$ denota o subintervalo de $\Sigma$ de raio $\delta$ (resp. $2 \delta$ ) centrado em $\bar{p}$.

Demonstração: A prova segue da Proposição 1.4 .5 e da igualdade $P\left(I_{\bar{p}}(2 \delta)\right)=\widetilde{P}\left(I_{\bar{p}}(2 \delta)\right)$.

Lema 1.4.7 Sejam $X \in \mathscr{C}$ e $\gamma$ uma trajetória positivamente recorrente não-trivial de $X$ satisfazendo ( $\star$. Sejam C um círculo transversal distinguido passando por $\bar{p} \in \gamma$, e $P: C \rightarrow C$ a correspondente transformação de primeiro retorno, tudo como na Proposição 1.3.13. Para cada $0<\lambda<1$, existe $\delta>0$ tal que o seguinte vale: para toda perturbação $\widetilde{P}$ de $P$ de classe $C^{r}$ localizada em $I_{\bar{p}}(\delta) \subset I_{\bar{p}}(2 \delta) \subset \operatorname{dom}(P)$ satisfazendo $\|\widetilde{P}-P\|_{C^{r}}<\delta$, e para cada intervalo $I \subset I_{\bar{p}}(2 \delta)$ satisfazendo

(i) $\ell(I)<\delta$

(ii) $\widetilde{P}^{n}(I)$ é um intervalo contido em $\operatorname{dom}(\widetilde{P})$ para todo $1 \leq n \leq L$, onde L $>0$ é um inteiro;

Temos que para cada $n^{*} \in\{1,2, \cdots, L\}$ satisfazendo $\widetilde{P}^{n^{*}}(I) \cap I_{\bar{p}}(\boldsymbol{\delta}) \neq \emptyset,\left.\quad \widetilde{P}^{n^{*}}\right|_{I} \quad$ é uma $\sqrt[16]{\lambda}$-contração infinitesimal. Além disso, $\widetilde{P}^{n^{*}}(I) \subset I_{\bar{p}}(2 \delta)$.

Demonstração: Seja $0<\lambda<1$ dado. Pela proposição 1.3 .13 , existe $N \in \mathbb{N}$ tal que $P^{n}$ é uma $\lambda$-contração infinitesimal para todo $n \geq N$. Necessitamos especificar quão pequeno $\delta>0$ tem de ser. Sejam $\bar{N}=N$ e $\rho>0$ como na Proposição 1.4.6, e assuma que $\delta<\rho$. Então para toda perturbação $\widetilde{P}$ de $P$ de classe $C^{r}$ localizada em $I_{\bar{p}}(\delta)$ temos que

$$
\widetilde{P}^{n}\left(I_{\bar{p}}(2 \delta)\right) \cap I_{\bar{p}}(2 \delta)=\emptyset \quad \text { para todo } \quad 1 \leq n \leq N-1
$$

Além disso, temos que $\operatorname{dom}(P)=\operatorname{dom}(\widetilde{P})$. Como $\|\widetilde{P}-P\|_{C^{r}}<\delta$, tomando $\delta>0$ ainda menor, podemos assumir que

$$
\sup _{x \in \operatorname{dom}(P)}|\log | D \widetilde{P}(x)|-\log | D P(x)||<\frac{|\log \lambda|}{64 N}<\frac{|\log \lambda|}{8 N} .
$$


Finalmente assumimos que $\delta>0$ é tão pequeno que a variação de $\log |D P|$ em cada intervalo $I_{\bar{p}}(2 \delta)$ é muito pequena; Mais precisamente, requeremos que para todo $x, y \in I_{\bar{p}}(2 \delta)$,

$$
|\log | D P(x)|-\log | D P(y)||<\frac{|\log \lambda|}{32 N} .
$$

Note que segue-se imediatamente da primeira desigualdade em (1.4) e de (1.5) que para todo $x, y \in I_{\bar{p}}(2 \delta)$,

$$
|\log | D \widetilde{P}(x)|-\log | D \widetilde{P}(y)||<\frac{|\log \lambda|}{16 N} .
$$

Pode indução finita podemos reduzir a prova do Lemma 1.4.7 ao seguinte caso. Para algum $n_{1} \in$ $\{0,1, \cdots, L\}$ e para algum $n * \in\left\{1,2, \cdots, L-n_{1}\right\}$, assuma que $I_{1}=\widetilde{P}^{n_{1}}(I) \subset I_{\bar{p}}(2 \delta) ; \widetilde{P}^{k}\left(I_{1}\right) \cap$ $I_{\bar{p}}(\delta)=\emptyset$, para todo $1 \leq k \leq n^{*}-1 ; \widetilde{P}^{n^{*}}\left(I_{1}\right) \cap I_{\bar{p}}(\delta) \neq \emptyset$; e $\ell\left(I_{1}\right)<\delta$. A prova estará terminada se $\left.\widetilde{P}^{n^{*}}\right|_{I_{1}}$ for uma $\sqrt[16]{\lambda}$-contração e $\widetilde{P}^{n^{*}}\left(I_{1}\right) \subset I_{\bar{p}}(2 \delta)$. Como $I_{1} \subset I_{\bar{p}}(2 \delta)$, segue-se de (1.3) que $n^{*} \geq N$. Assim, podemos escrever $n^{*}=r+k N$, onde $N \leq r<2 N$, e $k \in \mathbb{N}$. Além disso, como $P^{n}$ é uma $\lambda$-contração infinitesimal para todo $n \geq N$, temos pela Regra da Cadeia que para todo $y \in \operatorname{dom}\left(P^{n^{*}}\right)$

$$
\left|D P^{n^{*}}(y)\right|=\left|D P^{r}\left(P^{k N}(y)\right)\right|\left|D P^{k N}(y)\right|<\lambda^{k+1} .
$$

Consequentemente, em $\operatorname{dom}\left(P^{n *}\right)$, temos que (note que $\log \lambda<0$ )

$$
\frac{1}{n^{*}} \log \left|D P^{n^{*}}(y)\right|<\frac{(k+1) \log \lambda}{n^{*}}<\frac{\log \lambda}{4 N} .
$$

Pela definição de perturbação de classe $C^{r}$ de uma transformação de primeiro retorno, existe um difeomorfismo $G: \Sigma \rightarrow \Sigma$ de classe $C^{r}$ tal que $G$ é a aplicação identidade em $\Sigma \backslash I_{\bar{p}}(\delta), G\left(I_{\bar{p}}(\delta)\right) \subset$ $I_{\bar{p}}(\delta), \mathrm{e}$

$$
\widetilde{P}(x)=P(G(x)) \quad \text { para todo } \quad x \in \operatorname{dom}(P)
$$

Assim, como a perturbação está localizada em $I_{\bar{p}}(\delta)$, obtemos que

$$
\widetilde{P}^{k}(x)=P^{k-1}(\widetilde{P}(x)) \text { para todo } x \in I_{1} \cap \operatorname{dom}\left(P^{k}\right) \text { e para todo } 1 \leq k \leq n^{*}
$$

de maneira que de (1.9),

$$
\widetilde{P}^{k}(x)=P^{k}(y) \quad \text { para todo } \quad x \in I_{1} \cap \operatorname{dom}\left(P^{k}\right) \quad \text { e } \quad 1 \leq k \leq n^{*},
$$

onde $y=G(x) \in I_{\bar{p}}(2 \delta)$. 
Portanto, de (1.10) obtemos que para todo $x \in I_{1} \cap \operatorname{dom}\left(P^{n^{*}}\right)$,

$$
\begin{aligned}
\frac{1}{n^{*}} \log \left|D \widetilde{P}^{n^{*}}(x)\right| & =\frac{1}{n^{*}} \sum_{k=0}^{n^{*}-1} \log \left|D \widetilde{P}\left(\widetilde{P}^{k}(x)\right)\right|= \\
& =\frac{1}{n^{*}} \sum_{k=0}^{n^{*}-1} \log \left|D \widetilde{P}\left(P^{k}(y)\right)\right|+\frac{\log |D \widetilde{P}(x)|-\log |D \widetilde{P}(y)|}{n^{*}}
\end{aligned}
$$

onde $y=G(x) \in I_{\bar{p}}(2 \delta)$. Agora usando a segunda desigualdade em (1.4) e (1.8),

$$
\begin{array}{r}
\frac{1}{n^{*}} \sum_{k=0}^{n^{*}-1} \log \left|D \widetilde{P}\left(P^{k}(y)\right)\right|= \\
=\frac{1}{n^{*}} \sum_{k=0}^{n^{*}-1}\left(\log \left|D \widetilde{P}\left(P^{k}(y)\right)\right|-\log \left|D P\left(P^{k}(y)\right)\right|\right)+\frac{1}{n^{*}} \log \left|D P^{n^{*}}(y)\right| \\
<\frac{|\log \lambda|}{8 N}+\frac{\log \lambda}{4 N}=\frac{\log \lambda}{8 N} .
\end{array}
$$

Assim, usando (1.6) e (1.11), não é difícil mostrar que

$$
\frac{1}{n^{*}} \log \left|\left(D \widetilde{P}^{n^{*}}\right)(x)\right|<\frac{|\log \lambda|}{16 N}+\frac{\log \lambda}{8 N}=\frac{\log \lambda}{16 N}
$$

Finalmente, para todo $x \in I_{1} \cap \operatorname{dom}\left(P^{n^{*}}\right)$,

$$
\log \left|\left(D \widetilde{P}^{n^{*}}\right)(x)\right|<\frac{n^{*}}{N} \frac{\log \lambda}{16}<\frac{\log \lambda}{16}
$$

Logo $\left.P^{n^{*}}\right|_{I_{1}}$ é uma $\sqrt[16]{\lambda}$-contração infinitesimal. Agora como $\ell\left(I_{1}\right)<\delta$, temos que $\ell\left(\widetilde{P}^{n^{*}}\left(I_{1}\right)\right)<$ $\ell\left(I_{1}\right)<\delta$. Como $\widetilde{P}^{n^{*}}\left(I_{1}\right) \cap I_{\bar{p}}(\delta) \neq \emptyset$, temos que $\widetilde{P}^{n^{*}}\left(I_{1}\right) \subset I_{\bar{p}}(2 \delta)$.

Observação 1.4.8 Note que, contanto que a perturbação $\widetilde{P}$ de P seja pequena e o segmento transversal $I_{\bar{p}}(\delta)$ seja pequeno, a constante de contração $\sqrt[16]{\lambda}$ of $\left(\left.\widetilde{P}\right|_{I}\right)^{n^{*}}$ não depende nem do intervalo I, nem de $n^{*}$, nem da perturbação; ela depende somente da constante de contração $\lambda$ da transformação de primeiro retorno original $P$.

\subsection{Connecting Lema}

O primeiro passo na direção de obter-se trajetórias periódicas intersectando uma vizinhança $V_{p}$ de um ponto recorrente não-trivial $p$ é destruir todas as trajetórias recorrentes não-triviais inter- 
sectando $V_{p}$. O segundo passo é mostrar que, depois da perturbação, existe um segmento transversal $\Sigma$ passando por $p$ tal que a transformação de Poincaré $P: \Sigma \rightarrow \Sigma$ tem a propriedade de que $\cap_{n=1}^{\infty} \operatorname{dom}\left(P^{n}\right) \neq \emptyset$. Estes dois passos são tudo o que é necessário para provar um Closing Lemma Localizado de classe $C^{r}$ para a classe de campos vetoriais $\mathscr{C}$, o que implica um Closing Lemma para $\mathscr{C}$.

Relembramos que, pelo Teorema 1.3.6, sempre que existir uma trajetória recorrente não-trivial, também existe um par de separatrizes recorrentes não-triviais $\gamma_{1}, \gamma_{2}$ densas e contidas no conjunto quasiminimal $\mathrm{cl}(\gamma)$, e satisfazendo $\omega\left(\gamma_{1}\right) \cap \gamma_{2} \neq \emptyset$. Estes tipos de separatrizes são conhecidas como separatrizes não estabilizadas. Assim, uma maneira de se livrar de recorrência não-trivial ao redor de $p$ é conectar estas separatrizes por meio de perturbações twist locais. Isto é feito por meio de um Connecting Lema Fraco de classe $C^{r}$, o mais conhecido do qual sendo o Connecing Lemma de Peixoto. O próximo teorema é um passo intermediário na direção da prova do Teorema A. Ele inclui como caso particular o Teorema B.

Teorema 1.5.1 Sejam $X \in \mathscr{C}, \gamma$ uma trajetória de $X$ satisfazendo $(\star), p \in \gamma$ e $\varepsilon>0$. Sejam $C$ um círculo transversal distinguido passando por $\bar{p} \in \gamma$, onde $d(\bar{p}, p)<\varepsilon$, como na Proposição 1.3.13, e $Y \in \mathfrak{X}^{r}(M)$ uma perturbação de $X$ de classe $C^{r}$ localizada em $V_{\bar{p}}(\delta)$ tal que $\|\widetilde{P}-P\|_{C^{r}}<\delta$, onde $\delta>0$ é pequeno e $P$ (resp. $\widetilde{P})$ é a transformação de primeiro retorno induzida por $X$ (resp. $Y$ ) em C. Assuma que $Y$ tenha uma trajetória recorrente não-trivial $\widetilde{\gamma}$ acumulando-se em $\bar{p}$. Então Y pode ser arbitrariamente aproximado na topologia $C^{r}$ por um campo vetorial $Z$ que tem uma conexão de sela a mais que $Y$ e que é uma perturbação twist de classe $C^{r}$ de $Y$ (e assim uma perturbação de $X$ de classe $C^{r}$ ) localizada em $V_{\bar{p}}(\boldsymbol{\delta})$.

Demonstração: Seja $0<\lambda<1$ um número real satisfazendo a seguinte desigualdade:

$$
\frac{\sqrt[16]{\lambda}}{1-\sqrt[16]{\lambda}}<\frac{1}{16}
$$

Seja $\widetilde{P}: C \rightarrow C$ a transformação de primeiro retorno induzida por $Y$ em $C$. Note que por causa das hipóteses sobre $Y$ temos que $\widetilde{P}$ é uma perturbação de $P$ de classe $C^{r}$ localizada em $I_{\bar{p}}(\delta)$, a lateral esquerda de $V_{\bar{p}}(\delta)$. Escolhemos $\delta>0$ como no Lemma 1.4.7.

Pelo Teorema 1.3.6, existem uma separatriz instável $\gamma_{1}^{+}$de $Y$ e uma separatriz estável $\gamma_{2}^{-}$de $Y$, ambas trajetórias recorrentes não-triviais, tais que $\operatorname{cl}\left(\gamma_{1}^{+}\right)=\operatorname{cl}\left(\gamma_{2}^{-}\right)=\operatorname{cl}(\widetilde{\gamma})$. Assim, $\operatorname{cl}\left(\gamma_{1}^{+} \cap\right.$ $C)=\operatorname{cl}\left(\gamma_{2}^{-} \cap C\right)=\operatorname{cl}(\widetilde{\gamma} \cap C)$. Como $\widetilde{\gamma}$ se acumula em $\bar{p}$, existe $q \in \gamma_{2}^{-} \cap I_{\bar{p}}\left(\frac{\delta}{2}\right)$ e um intervalo $I_{q}\left(\delta^{\prime}\right) \subset I_{\bar{p}}(\delta)$ centrado em $q$ e não interceptando nenhuma das conexões de selas de $Y$, onde $\delta^{\prime}$ 
é muito menor que $\delta$. Iremos suportar a perturbação em uma caixa de fluxo especial $V_{q}\left(\delta^{\prime}\right)$ de $X$ para $I_{q}\left(\delta^{\prime}\right)$ de maneira que toda conexão de selas previamente existente não será destruída pela perturbação. (ver Figura 1.2).

Podemos escrever $I_{q}\left(\delta^{\prime}\right)-\{q\}=I_{q}^{-}\left(\delta^{\prime}\right) \cup I_{q}^{+}\left(\delta^{\prime}\right)$ como a união de suas duas componentes conexas $I_{q}^{-}\left(\delta^{\prime}\right)$ e $I_{q}^{+}\left(\delta^{\prime}\right)$, onde $I_{q}^{-}\left(\delta^{\prime}\right)=\left[q-\delta^{\prime}, q\right)\left(\right.$ resp. $\left.I_{q}^{+}\left(\delta^{\prime}\right)=\left(q, q+\delta^{\prime}\right]\right)$. Como $q$ é acumulada por $\gamma_{1}^{+}$, temos que $q$ é um ponto de aderência ou de $\gamma_{1}^{+} \cap I_{q}^{-}\left(\delta^{\prime}\right)$ ou de $\gamma_{1}^{+} \cap I_{q}^{+}\left(\delta^{\prime}\right)$. Sem perda de generalidade, podemos assumir que o primeiro caso acontece, o que geometricamente significa que $\gamma_{1}^{+}$acumula-se em $q$ por baixo (isto inclui o caso em que $q$ é acumulado por ambos os lados).

Relembramos que $V_{\bar{p}}(\boldsymbol{\delta})$ é uma caixa de fluxo especial de $X$ para $I_{\bar{p}}(\boldsymbol{\delta})$. Agora seja $V_{q}\left(\boldsymbol{\delta}^{\prime}\right)$ uma caixa de fluxo especial de $X$ para $I_{q}\left(\delta^{\prime}\right)$. Como $\delta^{\prime}$ é muito menor que $\delta$, a lateral esquerda $I_{q}\left(\delta^{\prime}\right)$ de $V_{q}\left(\delta^{\prime}\right)$ está contida na lateral direita $I_{\bar{p}}(\boldsymbol{\delta})$ de $V_{p}(\boldsymbol{\delta})$. Assumimos também que a lateral direita de $V_{q}\left(\boldsymbol{\delta}^{\prime}\right)$ está contida na lateral direita de $V_{\bar{p}}(\boldsymbol{\delta})$. Em particular, temos que $V_{q}\left(\boldsymbol{\delta}^{\prime}\right) \subset V_{\bar{p}}(\boldsymbol{\delta})$ (ver Figura 1.2).

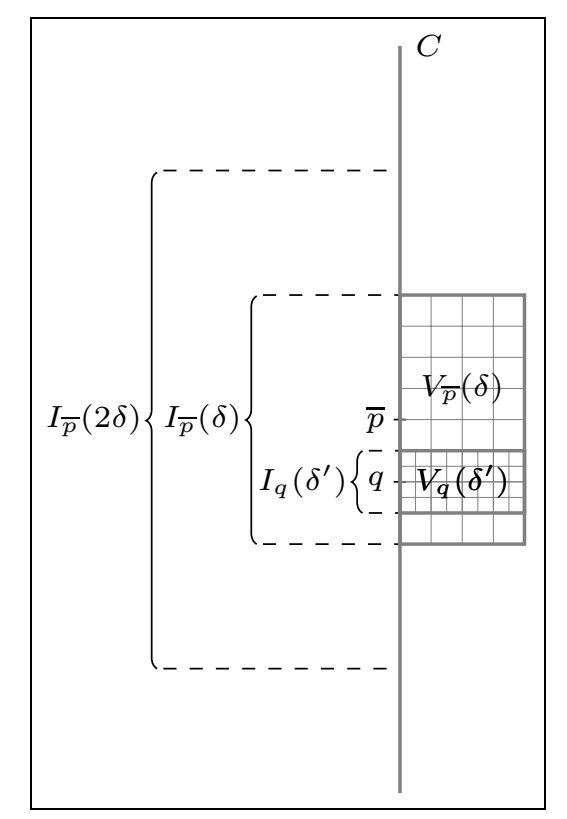

Figura 1.2: A caixa de perturbação.

Procedemos agora com a construção de uma família de perturbações twist de classe $C^{r}$ do campo vetorial $Y$ suportadas em $V_{q}\left(\delta^{\prime}\right) \subset V_{\bar{p}}(\boldsymbol{\delta})$. Seja $Y^{\perp}$ um campo vetorial unitário de classe $C^{r}$ definido em uma vizinhança aberta de $V_{q}\left(\delta^{\prime}\right)$ tal que $\left.Y^{\perp}\right|_{V_{q}\left(\delta^{\prime}\right)}$ é ortogonal a $\left.Y\right|_{V_{q}\left(\delta^{\prime}\right)}$ e aponta para cima. Seja $\xi: M \rightarrow[0,1]$ uma função bacia de classe $C^{\infty}$ suportada em $V_{q}\left(\delta^{\prime}\right)$. Seja $\left\{Y_{s}\right\}_{s \in[0, \sigma]}$ a família a um parâmetro de perturbações twist de classe $C^{r}$ de $Y$ suportadas em $V_{q}\left(\delta^{\prime}\right) \subset V_{\bar{p}}(\delta)$ e definidas por

$$
Y_{s}=Y+s k \xi Y^{\perp}
$$




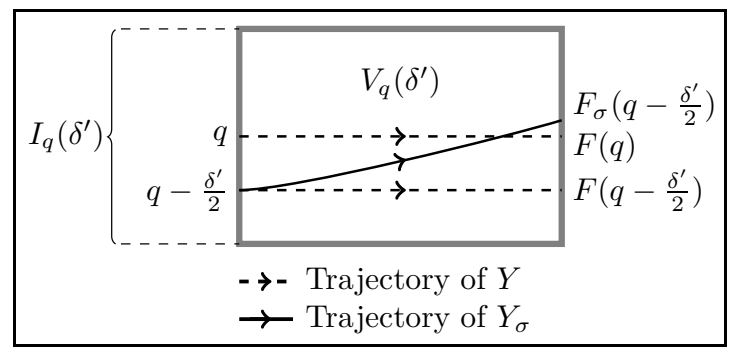

Figura 1.3: Minimum lift property.

onde $k$ é uma constante positiva (o fator de inclinação). Doravante, omitiremos o subscrito dos termos respectivos sempre que $s=0$. Desta maneira, os símbolos $Y, F, \widetilde{\phi} \ldots$ irão significar os símbolos $Y_{0}, F_{0}, \widetilde{\phi}_{0} \ldots$ respectivamente.

Escolhendo $k$ grande o bastante, podemos assumir que há uma zona $V_{q}\left(\frac{\delta^{\prime}}{2}\right)$ centrada em $q$ de raio $\frac{\delta^{\prime}}{2}$ onde o campo vetorial $Y_{\sigma}$ experimenta um grande desvio. De uma maneira mais precisa, seja $F_{S}$ a transformação de Poincaré que leva a lateral esquerda $I_{\bar{p}}(\delta)$ de $V_{\bar{p}}(\delta)$ na lateral direita de $V_{\bar{p}}(\delta)$ induzida pelo campo vetorial $Y_{s}$. Munimos a lateral esquerda $I_{\bar{p}}(\delta) \subset C$ de $V_{\bar{p}}(\delta)$ com a orientação induzida por $C$ e colocamos na lateral direita de $V_{\bar{p}}(\delta)$ a orientação induzida pela transformação de Poincaré $F_{S}$ (esta orientação não depende de $s$ ). Ajustando o fator de inclinação $k$, podemos assumir que $F_{\sigma}(x)>F(q)$, para todo $x \in I_{q}\left(\frac{\delta^{\prime}}{2}\right)$ (ver Figura 1.3). Referimo-nos a isto como "minimum lift property"de Peixoto.

Sejam $\widetilde{\phi}^{s}: M \times \mathbb{R} \rightarrow \mathbb{R}$ o fluxo associado com $Y_{S}$ e $\widetilde{P}_{S}: C \rightarrow C$ a transformação de primeiro retorno induzida por $\widetilde{\phi}^{s}$ em $C$. Como $\alpha\left(\gamma_{1}^{+}\right)$é uma sela, existe $q_{0} \in \gamma_{1}^{+} \cap I_{q}\left(\delta^{\prime}\right)$ tal que $\left\{\widetilde{\phi}\left(t, q_{0}\right) \mid t \in\right.$ $\left.\mathbb{R}_{-}\right\} \cap I_{q}\left(\delta^{\prime}\right)=\left\{q_{0}\right\}$, onde $\widetilde{\phi}$ é o fluxo gerado por $Y$. Considere a região $R$ definida por

$$
R=\left\{\widetilde{\phi}^{s}\left(t, q_{0}\right), s \in[0, \sigma] \text { e } t \geq 0\right\}
$$

Se $R$ contiver selas de $Y$ então a prova estará terminada. De fato, por continuidade, para algum $s \in[0, \sigma]$, a semitrajetória positiva de $Y_{s}$ iniciando-se em $q_{0}$ iria convergir para esta sela. Logo podemos assumir a seguinte hipótese:

(H) A região $R$ não contém nenhuma sela de $Y$.

Desta maneira, por $(i i i)$ da Proposição 1.3.13, todas as semitrajetórias positivas de $Y_{s}, s \in[0, \sigma]$, iniciando-se em $q_{0}$ intersectam $C$ infinitas vezes. Para cada $n \in \mathbb{N}$, sejam $I_{n}=\left\{\left(\widetilde{P}_{s}\right)^{n}\left(q_{0}\right) \mid s \in[0, \sigma]\right\}$, 
e $\Omega=\left\{n \in \mathbb{N}: I_{n} \cap I_{q}\left(\delta^{\prime}\right) \neq \emptyset\right\}$, onde $I_{0}=\left\{q_{0}\right\}$. Podemos escrever $\Omega=\left\{n_{j}\right\}_{j=0}^{\infty}$ como uma sequiência crescente de inteiros positivos. Segue-se por continuidade que cada $I_{n}$ é um conjunto fechado conexo, isto é, um subintervalo de $C$. Ainda, como a perturbação $\widetilde{P}_{S}$ de classe $C^{r}$ de $P$ está localizada em $I_{q}\left(\delta^{\prime}\right)$ temos que $\widetilde{P}_{s}(x)=\widetilde{P}(x)$ para todo $s \in[0, \sigma]$ e $x \in C \backslash I_{q}\left(\delta^{\prime}\right)$. Consequentemente, paa todo $n \notin \Omega$,

$$
I_{n+1}=\widetilde{P}\left(I_{n}\right)
$$

Segue-se de (H) que para todo $n \notin \Omega$,

$$
I_{n} \subset \operatorname{dom}(\widetilde{\mathrm{P}})
$$

Como $\widetilde{P}_{S}$ é uma perturbação twist de classe $C^{r}$ de $P$ localizada em $I_{q}\left(\delta^{\prime}\right)$, temos que existe uma sequência de subintervalos $\left\{I_{n}^{\prime}\right\}_{n=0}^{\infty}$ de $C$ tais que $I_{n}^{\prime}=I_{n}$, para todo $n \notin \Omega$, e para todo $n \in \mathbb{N}$ o seguinte vale:

$$
I_{n}^{\prime} \subset \operatorname{dom}(\widetilde{P}), \quad I_{n+1}^{\prime}=\widetilde{P}\left(I_{n}^{\prime}\right), \quad \ell\left(I_{n}^{\prime}\right) \leq \ell\left(I_{n}\right)+2 \delta^{\prime}
$$

Pelo Lema 1.4.7, e pelo fato que $I_{0}^{\prime} \subset I_{q}\left(\delta^{\prime}\right) \subset I_{q}\left(2 \delta^{\prime}\right) \subset I_{\bar{p}}(2 \delta)$ e $\ell\left(I_{q}\left(\delta^{\prime}\right)\right)<2 \delta^{\prime}$, temos que para todo $j \in \mathbb{N}, I_{n_{j}} \subset I_{\bar{p}}(2 \delta)$, e

$$
\ell\left(I_{n_{j+1}}\right)<\sqrt[16]{\lambda} \ell\left(I_{n_{j}}^{\prime}\right)<\sqrt[16]{\lambda}\left(\ell\left(I_{n_{j}}\right)+2 \delta^{\prime}\right)
$$

Por indução e usando (1.13), podemos mostrar que para todo $j \in N$,

$$
\ell\left(I_{n_{j}}\right)<2 \delta^{\prime} \frac{\sqrt[16]{\lambda}}{1-\sqrt[16]{\lambda}}<\frac{\delta^{\prime}}{4}
$$

Como $\left\{\widetilde{P}^{n}\left(q_{0}\right)\right\}_{n=0}^{\infty}$ acumula-se em $q$ por baixo, tomando uma subsequência se necessário, podemos assumir que $\widetilde{P}^{n_{j}}\left(q_{0}\right) \uparrow q$. Assim, existe $K>0$ tal que para todo $j \geq K$,

$$
q-\widetilde{P}^{n_{j}}\left(q_{0}\right)<\frac{\delta^{\prime}}{4}
$$

De (2.4), como $\left\{\widetilde{P}^{n_{j}}\left(q_{0}\right),\left(\widetilde{P}_{\sigma}\right)^{n_{j}}\left(q_{0}\right)\right\} \subset I_{n_{j}}$, temos que para todo $j \in N$,

$$
\widetilde{P}^{n_{j}}\left(q_{0}\right)-\left(\widetilde{P}_{\sigma}\right)^{n_{j}}\left(q_{0}\right)<\ell\left(I_{n_{j}}\right)<\frac{\delta^{\prime}}{4}
$$


Assim,

$$
\left(\widetilde{P}_{\sigma}\right)^{n_{j}}\left(q_{0}\right)-q<\frac{\delta^{\prime}}{2}
$$

Agora pela minimum lift property, existe algum $s \in[0, \sigma]$ tal que a semitrajetória positiva de $Y_{s}$ iniciando-se em $q_{0}$ converge para uma sela hiperbólica. Esta contradição mostra que $(\mathrm{H})$ é falso, o que implica o resultado. Mais especificamente, existe $s \in[0, \sigma]$, e $Z=Y_{s}$ tendo uma conexão de sela a mais que $Y$.

Observação 1.5.2 Note que na prova do Teorema 1.5.1 podemos construir a família de perturbações $\left\{Y_{s}\right\}_{s \in[0, \sigma]}$ de tal maneira que $\left\|Y_{s}-Y\right\|_{C^{r}}<\eta$, para todo $s \in[0, \sigma]$, onde $\eta$ é qualquer constante positiva previamente dada. Em particular, se escolhermos $\eta<\delta$ podemos aplicar o Teorema 1.5.1 outra vez ao novo campo vetorial $Z$ e assim por diante.

Teorema 1.5.3 Sejam $X \in \mathscr{C}, \gamma$ uma trajetória de $X$ satisfazendo $(\star)$, e $p \in \gamma$. Para cada $\varepsilon>0$, existem $\bar{p} \in \gamma$, com $d(\bar{p}, p)<\varepsilon$, uma vizinhança $V_{\bar{p}}$ de $\bar{p}$, e $Y \in \mathfrak{X}^{r}(M)$ arbitrariamente próximo de $X$ na topologia $C^{r}$ tal que nenhuma trajetória recorrente não-trivial de $Y$ intersecta $V_{\bar{p}}$.

Demonstração: A prova segue-se pela aplicação do Teorema 1.5.1 e da Observação 1.5.2 um número finito de vezes.

\subsection{Closing Lema}

Teorema 1.6.1 Sejam $X \in \mathscr{C}, \gamma$ uma trajetória de $X$ satisfazendo $(\star)$, e $p \in \gamma$. Então para cada $\varepsilon>0$, existem $\bar{p} \in \gamma$, com $d(\bar{p}, p)<\varepsilon$, uma sequência $\left\{X_{n}\right\}_{n=1}^{\infty}$ de campos vetoriais de classe $C^{r}$ tendendo a X na topologia $C^{r}$, e uma sequência de pontos $\left\{\bar{p}_{n}\right\}_{n=1}^{\infty}$ tendendo a $\bar{p}$ tal que $\bar{p}_{n}$ é um ponto periódico de $X_{n}$ para cada $n \in \mathbb{N}$.

Demonstração: Pelo Teorema 1.5.3, existem um segmento transversal $\Sigma_{\bar{p}}$ contendo $\bar{p}$ e um campo vetorial $Y$ arbitrariamente próximo de $X$ na topologia $C^{r}$ sem trajetórias recorrente não-triviais intersectando $\Sigma_{\bar{p}}$. Além disso, $Y$ é uma perturbação local de classe $C^{r}$ de $X$ de maneira que por (iii) da Proposição 1.3.13, existem pontos em $\Sigma_{\bar{p}}$ cujas trajetórias positivas intersectam $\Sigma_{\bar{p}}$ um número infinito de vezes. Seja $x_{0} \in \Sigma_{\bar{p}}$ qualquer destes pontos. Como $\gamma_{x_{0}}^{+}$não é uma trajetória recorrente não-trivial, temos que $\omega\left(\gamma_{x_{0}}^{+}\right)$é ou uma trajetória periódica ou um grafo, cada um dos 
quais intersectando $\Sigma_{\bar{p}}$. Em qualquer caso, podemos aproximar $Y$ arbitrariamente por um campo vetorial de classe $C^{r}$ tendo uma trajetória periódica intersetando $\Sigma_{\bar{p}}$. Para obter uma sequência de pontos periódicos tendendo a $\bar{p}$ considere uma sequência encaixada de segmentos $\left\{\Sigma_{n}\right\}_{n=1}^{\infty}$, com $\Sigma_{n} \supset \Sigma_{n+1}$, tais que $\{\bar{p}\}=\cap_{n=1}^{\infty} \Sigma_{n}$. Então podemos aplicar o argumento anterior para cada $\Sigma_{n}$ para obter uma trajetória periódica de um campo vetorial $X_{n}$ de classe $C^{r}$ que intercepta $\Sigma_{n}$, onde $X_{n} \rightarrow X$ na topologia $C^{r}$ quando $n \rightarrow \infty$.

Lema 1.6.2 Sejam $\left\{\bar{p}_{n}\right\}_{n=1}^{\infty}, \bar{p}$, e $\left\{X_{n}\right\}_{n=1}^{\infty}$ como no Teorema 1.6.1. Então existe uma sequência de campos vetoriais $\left\{Y_{n}\right\}_{n=1}^{\infty}$ de classe $C^{r}$ tendendo a $X$ na topologia $C^{r}$ tal que $\bar{p}$ é um ponto periódico de $Y_{n}$.

Demonstração: Sob as hipóteses e conclusões do Teorema 1.6.1, existe uma sequência $F_{n}: M \rightarrow M$ de difeomorfismos de classe $C^{r}$ tal que $F_{n}\left(\bar{p}_{n}\right)=\bar{p}$. Podemos assumir que $F_{n}$ converge para a identidade no topologia $C^{r+1}$. Portanto, a sequência de campos vetoriais $Y_{n}=F_{n *}\left(X_{n}\right)$ converge para $X$ na topologia $C^{r}$ e cada $F_{n *}\left(X_{n}\right)$ tem uma trajetória periódica passando por $\bar{p}$.

Teorema A. Sejam $M$ uma variedade bidimensional compacta, $r \geq 2$ um inteiro, $X \in \mathscr{C}, \gamma$ uma trajetória de $X$ satisfazendo $(\star)$, e $p \in \gamma$. Existe $Y \in \mathfrak{X}^{r}(M)$ arbitrariamente próximo de $X$ na topologia $C^{r}$ tendo uma trajetória periódica passando por $p$.

Demonstração: Pelo Teorema 1.6.1 e Lema 1.6.2, existem uma sequência de pontos $\bar{p}_{n} \rightarrow p$, e uma sequência $X_{n}$ de campos vetoriais de classe $C^{r}$ convergindo para $X$ na topologia $C^{r}$ tal que cada $\bar{p}_{n}$ é um ponto periódico $X_{n}$. Procedendo como na prova do Lema 1.6.2, podemos construir uma sequência $\left\{Y_{n}\right\}_{n=0}^{\infty}$ de campos vetoriais de classe $C^{r}$ convergindo para $X$ na topologia $C^{r}$ tal que $\bar{p}$ é um ponto periódico de $Y_{n}$.

As seções seguintes são devotadas para a prova do Teorema C. Na Seção 1.7 introduzimos um pouco de teoria da medida que será necessário adiante. Na Seção 1.8 explicamos o que significa requerer que o divergente de um campo vetorial nas suas selas seja menor ou igual a zero. $\mathrm{Na}$ Seção 1.9, usando um pouco de Teoria Ergódica, provamos o Teorema $C$. 


\subsection{Medida Transversal e Transformações de Primeiro Retorno}

Seja $X \in \mathfrak{X}^{r}(M)$ um campo vetorial de classe $C^{r}$ que tem um número finito de singularidades, todas hiperbólicas, e uma trajetória recorrente não-trivial $\gamma$. Sejam $\Sigma$ um segmento transversal ou círculo transversal passando por $\gamma$ e $P: \Sigma \rightarrow \Sigma$ a correspondente transformação de primeiro retorno. Munimos $\Sigma$ com a estrutura $(\Sigma, \mathscr{B}, v)$ de um espaço de medida de Borel, onde $\mathscr{B}$ é a $\sigma$-algebra de Borel em $\Sigma$, e $v: \mathscr{B} \rightarrow[0,1]$ é a medida de probabilidade de Borel em $\Sigma$. Dizemos que um conjunto de Borel $W \subset \operatorname{dom}(P)$ (resp. $W \subset \operatorname{dom}\left(P^{-1}\right)$ ) é positivamente invariante (resp. negativamente invariante) se $P(W) \subset W$ (resp. $\left.P^{-1}(W) \subset W\right)$. Um conjunto de Borel $W \subset \Sigma$ é denominado invariante se $W$ é positivamente e negativamente invariante. Como um subconjunto de $(\Sigma, \mathscr{B}, v), W$ herda a estrutura de espaço de medida. Com algum abuso de notação, denotamos a restrição $\left.P\right|_{W}: W \rightarrow W$ por $P$. O maior conjunto positivamente (resp. negativamente) invariante é o conjunto $\Omega_{+}$(resp. $\Omega_{-}$) de pontos cujas semitrajetórias positivas (resp. negativas) intersectam $\Sigma$ infinitas vezes. Mais precisamente:

$$
\Omega_{+}=\bigcap_{n=1}^{\infty} \operatorname{dom}\left(P^{n}\right) \text { e } \quad \Omega_{-}=\bigcap_{n=1}^{\infty} \operatorname{dom}\left(P^{-n}\right) .
$$

Dizemos que $P$ preserva a medida $v$, ou que $v$ é invariante por $P$, ou que $v$ é $P$-invariante se $v\left(P^{n}(B)\right)=v(B)$, para cada $n \in \mathbb{Z}$ e $B \in \mathscr{B}$. Sem perda de generalidade, sempre assumiremos que os segmentos transversais e círculos transversais são disjuntos de trajetórias periódicas e conexões de selas. Neste caso, toda medida de probabilidade de Borel $P$-invariante é uma medida transversal (ver a Introdução para a definição de medida transversal).

Uma transformação de primeiro retorno $P: \Sigma \rightarrow \Sigma$ é unicamente ergódica se a medida transversal $v$ em $\Sigma$ é a única medida de probabilidade de Borel invariante por $P$. Usando isomorfismos canônicos, podemos transportar esta propriedade a todos os segmentos transversais contidos em uma vizinhança de $\gamma$. Uma medida de probabilidade de Borel $v$ invariante por $P$ é ergódica se sempre que $P^{-1}(B)=B$ para algum conjunto de Borel $B$ tivermos que ou $v(B)=0$ ou $v(B)=1$.

Para tirar vantagem de alguns resultados importantes de teoria ergódica é necessário que a transformação de primeiro retorno $P$ possa ser estendida a um conjunto compacto positivamente invariante (resp. negativamente invariante). Como a próxima proposição mostra, uma transformação de primeiro retorno sempre admite uma boa extensão.

Proposição 1.7.1 Seja $X \in \mathfrak{X}^{r}(M)$ um campo vetorial de classe $C^{r}$ que tem singularidades, to- 
das hiperbólicas, e uma trajetória recorrente não-trivial $\gamma$. Sejam C um círculo transversal passando por $\gamma$ e $P: C \rightarrow C$ a correspondente transformação de primeiro retorno. Assuma que $P$ seja unicamente ergódica. Existe uma extensão unicamente ergódica $\bar{P}$ de P para $\mathrm{cl}\left(\Omega_{+}\right)$tal que $\bar{P}\left(\operatorname{cl}\left(\Omega_{+}\right)\right) \subset \operatorname{cl}\left(\Omega_{+}\right)$.

Demonstração: Primeiramente notemos que $\Omega_{+}=\cap_{n=1}^{\infty} \operatorname{dom}\left(P^{n}\right)$ é um conjunto positivamente invariante. Pelo Teorema 1.2.2, $\partial \operatorname{dom}(P)$ é um conjunto finito e como $\operatorname{cl}\left(\Omega_{+}\right) \backslash \operatorname{dom}(P) \subset \partial \operatorname{dom}(P)$, temos que $P$ está definida em todos os pontos de $\operatorname{cl}\left(\Omega_{+}\right)$excetuando-se um número finito deles. Desta maneira qualquer tranformação $\bar{P}: \operatorname{cl}(\operatorname{dom}(P)) \rightarrow C$ satisfazendo que $\left.\bar{P}\right|_{\operatorname{dom}(P)}=\left.P\right|_{\operatorname{dom}(P)}$ e $\bar{P}(\partial \operatorname{dom}(P)) \subset \Omega_{+}$será uma extensão unicamente ergódica de $P$ tal que $\bar{P}\left(\operatorname{cl}\left(\Omega_{+}\right)\right) \subset \operatorname{cl}\left(\Omega_{+}\right)$.

\subsection{Sobre o divergente de um campo vetorial nas suas selas}

Em um campo vetorial planar apresentando um número finito de singularidades, todas hiperbólicas, a dinâmica das transformações de primeiro retorno associadas com o campo vetorial é, em grande parte, determinada pela dinâmica do sistema ao redor de suas selas hiperbólicas. A situação é mesmo melhor porque estaremos lidando somente com campos vetoriais de classe $C^{2}$. Neste caso um resultado de Hartman [31] nos permite assumir que todas as selas são lineares.

Mostraremos que se $P$ é uma transformação de primeiro retorno de um campo vetorial de classe $C^{2}$ então $\log |D P|$ é limitada superiormente (resp. inferiormente) contanto que o divergente de cada sela seja menor ou igual a zero (resp. maior ou igual a zero). Além disso, veremos que sob algumas hipóteses adicionais podemos substituir a função $\log |D P|$ por uma função escada (i.e. uma função constante por partes).

Como os principais resultados desta seção são assumidos serem conhecidos pela comunidade matemática, iremos omitir suas provas. Como referência indicamos [44].

Proposição 1.8.1 Seja $X \in \mathfrak{X}^{r}(M), r \geq 2$, um campo vetorial de classe $C^{r}$ que tem singularidades, todas hiperbólicas. Assuma que o divergente de X em cada uma de suas selas seja menor ou igual a zero (resp. maior ou igual a zero). Sejam $\Sigma$ um segmento transvesal ou um círculo transversal a $X$ e $P: \Sigma \rightarrow \Sigma$ a correspondente transformação de primeiro retorno cujo domínio é assumido ser não-vazio. Então $\log |D P|$ é limitada superiormente (resp. limitada inferiormente). Além disso, $\log |D P(x)|$ tende a $-\infty$ ou a uma constante (resp. $\log |D P(x)|$ tende a $+\infty$ ou a uma constante) quando $x$ tende monotonamente a $p \in \partial \operatorname{dom}(P)$. 
No próximo teorema por função escada entendemos uma função constante por partes, ou mais especificamente, uma função que é a suma de um número finito de funções indicadoras de intervalos disjuntos dois a dois. Relembramos que uma função indicadora de um conjunto $U$ assume o valor 1 em $U$, e o valor 0 em $X \backslash U$. Note que como a medida transversal se anula no complemento de $\operatorname{dom}(P)$, podemos supor que a função escada está definida em todo o círculo transversal $C$.

Teorema 1.8.2 Sejam $X \in \mathfrak{X}^{r}(M), r \geq 2$, um campo vetorial de classe $C^{r}$ que tem singularidades, todas hiperbólicas, $C$ um círculo transversal especial a $X, P: C \rightarrow C$ a correspondente tranformação de primeiro retorno que é assumidada ser unicamente ergódica, e $v$ a única medida de probabilidade de Borel invariante por $P$.

(i) Se o divergente de X em cada uma de suas selas é menor ou igual a zero e $\int \log |D P| \mathrm{d} v<-c$ para algum $c>0$, existem $N \in \mathbb{N}$ e uma sequência monótona $\left\{\varphi_{n}\right\}_{n=0}^{\infty} \downarrow \log |D P|$ de funções escadas definidas em $\operatorname{dom}(P)$ tais que $\int \varphi_{n} \mathrm{~d} v<-\frac{c}{2}$ para todo $n>N$;

(ii) Se o divergente de $X$ em cada uma de suas selas é maior ou igual a zero e $\int \log |D P| \mathrm{d} v>c$ para algum $c>0$, existem $N \in \mathbb{N}$ e uma sequência monótona $\left\{\psi_{n}\right\}_{n=0}^{\infty} \uparrow \log |D P|$ de funções escada definidas em $\operatorname{dom}(P)$ tais que $\int \psi_{n} \mathrm{~d} v>\frac{c}{2}$ para todo $n>N$.

\subsection{O caso unicamente ergódico}

Nesta seção provaremos o Teorema C. Vamos precisar do seguinte resultado que diz que na Definição 0.0.4 podemos supor que o segmento transversal é um círculo transversal especial.

Proposição 1.9.1 Sejam $X \in \mathscr{H}, \gamma$ uma trajetória de $X$ com as propriedades $(a)-(c), C$ um círculo transversal especial passando por $\gamma$ e $P: C \rightarrow C$ a correspondente transformação de primeiro retorno. Então existe uma constante $c>0$ tal que $\int \log |D P| \mathrm{d} v<-c$, onde $v$ é a única medida de probabilidade de Borel invariante por $P$.

Demonstração: Por hipótese, existem um segmento transversal $\Sigma$ passando por $\gamma$ e uma constante $c>0$ tal que a correspondente transformação de primeiro retorno $P_{\Sigma}: \Sigma \rightarrow \Sigma$ é $v_{\Sigma}-$ quaseintegrável e $\int P_{\Sigma} \mathrm{d} v_{\Sigma}<-c$, onde $v_{\Sigma}$ é a única medida de probabilidade de Borel em $\Sigma$ invariante por $P_{\Sigma}$. Usando transformações de Poincaré apropriadas, podemos assumir que $\Sigma$ está contido em um círculo transversal especial $C$ passando por $\gamma$. Note que à medida $v_{\Sigma}$ está associada uma 
única medida de probabilidade de Borel $v$ em $C$ que é invariante pela tranformação de primeiro retorno $P: C \rightarrow C$ induzida por $X$ em $C$. Pelo Teorema 1.8.2 podemos encontrar uma função escada $\varphi>\log |D P|$ definida em $\operatorname{dom}\left(P_{\Sigma}\right) \subset \Sigma$ tal que $\int \varphi \mathrm{d} \nu_{\Sigma}<-\frac{c}{2}$. Pelo Teorema Ergódico de Birkhoff, existe um conjunto $B_{\Sigma} \subset \Sigma$ positivamente $P_{\Sigma}$-invariante tal que $v_{\Sigma}\left(B_{\Sigma}\right)=1$ e para todo $x \in B_{\Sigma}$ :

$$
\liminf _{n \rightarrow \infty} \frac{1}{n} \sum_{k=0}^{n-1}\left|D P\left(P^{k}(x)\right)\right| \leq \lim _{n \rightarrow \infty} \frac{1}{n} \sum_{k=0}^{n-1} \varphi\left(P^{k}(x)\right)=\int \varphi \mathrm{d} v_{\Sigma}<-\frac{c}{2}
$$

Seja $\beta:(\mathbb{N} \backslash\{0\}) \times B_{\Sigma} \rightarrow \mathbb{N}$ a função definida por:

$$
P_{\Sigma}^{n}(x)=P^{\beta(n, x)}(x)
$$

É claro que $\beta$ é uma função localmente constante em $x$. Conseqüentemente, para todo $x \in B_{\Sigma} \mathrm{e}$ $n \in \mathbb{N} \backslash\{0\}:$

$$
D P_{\Sigma}^{n}(x)=D P^{\beta(n, x)}(x)
$$

Além disso, temos que $\beta(n, x) \geq n$ para todo $x \in B_{\Sigma}$ e $n \in \mathbb{N}$. Um simples argumento combinatório resulta na seguinte igualdade para todo $x \in B_{\Sigma}$ e $n \in \mathbb{N} \backslash\{0\}$ :

$$
\beta(n, x)=\sum_{i=0}^{n-1} \beta\left(1, P_{\Sigma}^{i}(x)\right)
$$

Note que $\beta(1, y)-1$ é o número de intersecções do círculo $C$ com o arco de trajetória de $X$ conectando y a $P_{\Sigma}(y)$. Segue-se dos argumentos de dinâmica topológica que tal número é finito e constante em cada componente conexa de $\operatorname{dom}\left(P_{\Sigma}\right)$ (ver [50, p. 146]). Assim existe uma cosntante $K>0$ tal que para todo $x \in B_{\Sigma}$ e $n \in \mathbb{N} \backslash\{0\}$ :

$$
\beta(n, x) \leq n K
$$

Segue-se do que foi feito acima que para todo $x \in B_{\Sigma}$ :

$$
\begin{aligned}
\liminf _{r \rightarrow \infty} \frac{1}{r} \log \left|D P^{r}(x)\right| & \leq \liminf _{n \rightarrow \infty} \frac{1}{\beta(n, x)} \log \left|D P^{\beta(n, x)}(x)\right| \\
& =\liminf _{n \rightarrow \infty} \frac{1}{n} \log \left|D P_{\Sigma}^{n}(x)\right| \cdot \frac{n}{\beta(n, x)} \\
& \leq \frac{1}{K} \liminf _{n \rightarrow \infty} \frac{1}{n} \log \left|D P_{\Sigma}^{n}(x)\right|<0 .
\end{aligned}
$$


É claro que (1.17) também vale para todo ponto $x \in \cup_{n=0}^{\infty} P^{-n}\left(B_{\Sigma}\right)$, que é um conjunto de medida total em $C$. Pela Proposição 1.8.1, $\log ^{+}|D P|$ é integrável. Este fato, juntamente com (1.17), implica que existe uma constante $c^{\prime}>0$ tal que $\int \log |D P| \mathrm{d} v<-c^{\prime}$.

Seja $X \in \mathfrak{X}^{r}(M)$ um campo vetorial de classe $C^{r}$ que tem singularidades, todas hiperbólicas. Dado um círculo transversal especial $C$ a $X$, denotamos por $P: C \rightarrow C$ a correspondente transformação de primeiro. Em cada ponto de $\operatorname{dom}\left(P^{n}\right)$ definimos as taxas médias de expansão para $P$ como sendo:

$$
\rho_{n}(x)=\frac{1}{n} \sum_{k=0}^{n-1} \log \left|D P\left(P^{k}(x)\right)\right|
$$

Definição 1.9.2 ( $\lambda$-contração infinitesimal $\mu$-expansão infinitesimal) $\quad$ Sejam $P: C \rightarrow C$ uma transformação de primeiro retorno suave, $n \in \mathbb{N}, A \subset \operatorname{dom}\left(P^{n}\right)$ e $0<\lambda<1$ (resp. $\left.\mu>1\right)$. Dizemos que $P^{n} \mid$ A é uma $\lambda$-contração infinitesimal (resp. $\mu$-expansão infinitesimal) se $\left|D P^{n}(x)\right|<\lambda$ (resp. $\left.\left|D P^{n}(x)\right|>\mu\right)$ para todo $x \in A$.

Observação 1.9.3 Note que, substituindo $P$ por $P^{-1}$ nas expressões anteriores, todas as definições dadas até aqui fazendo sentido para $n \in \mathbb{Z}_{-}=\{0,-1,-2, \ldots\}$.

Proposição 1.9.4 Seja $P: C \rightarrow C$ uma transformação de primeiro retorno suave. Dado $0<\lambda<1$, assuma que existam $d>0, n \in \mathbb{N}$, e $A \subset \operatorname{dom}\left(P^{n}\right)$ tais que $e^{-n d}<\lambda$ e $\rho_{n}(x)<-d$, para todo $x \in A$. Então o seguinte vale:

$$
P^{n} \mid \text { A é uma } \lambda \text {-contração infinitesimal. }
$$

Demonstração: É fácil ver que $\rho_{n}(x)=\log \sqrt[n]{\left|D P^{n}(x)\right|}$, para todo $x \in \operatorname{dom}\left(P^{n}\right)$. Por hipótese, $\rho_{n}(x)<-d$ para todo $x \in A$. Assim, $\left|D P^{n}(x)\right|<e^{-d n}<\lambda$ para todo $x \in A$.

Proposição 1.9.5 Seja $P: C \rightarrow C$ uma transformação de primeiro retorno suave. Dado $\mu>1$, assuma que existam $d>0$ e $n \in \mathbb{N}$ tais que $e^{n d}>\mu$ e $\rho_{-n}(x)>d$ para todo $x \in \Omega_{-}$. Então:

$$
\left.P^{-n}\right|_{\Omega_{-}} \text {é uma } \mu \text {-expansão infinitesimal. }
$$

Demonstração: A prova é similar àquela da proposição anterior. 1.9.4. 
Uma boa abordagem para obter um limitante superior (resp. limitante inferior) para as taxas médias de expansão para $P$ (resp. $P^{-1}$ ) é substituir $\log |D P|\left(\right.$ resp. $\left.\log \left|D P^{-1}\right|\right)$ em (1.18) por uma função escada $\varphi$ (resp. $\psi$ ) tal que $\log |D P|<\varphi$ (resp. $\log \left|D P^{-1}\right|>\varphi$ ), e então estudar as respectivas médias de Birkhoff para a função $\varphi$ (resp. $\psi$ ). Relembramos que, sob algumas hipóteses adicionais, o Teorema 1.8.2 nos permite fazer isso. Agora desenvolvemos um pouco de teoria ergódica para funções escada. É de importância fundamental notar que precisamos de resultados que valham em todos os pontos e não apenas num conjunto de medida total.

Na próxima proposição, $\chi_{U}$ denota a função indicadora do conjunto $U$, isto é, $\chi_{U}: X \rightarrow \mathbb{R}$ assume o valor 1 em $U$, e 0 em $X \backslash U$. Como usualmente, $\partial U$ denota a fronteira topológica de $U$.

Lema 1.9.6 Sejam $X=(X, \mathscr{B})$ um espaço mensurável compacto de Borel, e $v$ a única medida de probabilidade de Borel invariante por uma aplicação unicamente ergódica $f: X \rightarrow X$. Então para todo conjunto aberto $U \subset X$ tal que $v(\partial U)=0$ temos que as médias de Birkhoff $(1 / n) \sum_{k=0}^{n-1} \chi_{U}\left(f^{k}(x)\right)$ convergem uniformemente para $v(U)$.

Demonstração: Ver Katok e Hasselblatt [40, Corollary 4.1.14, p. 140].

Proposição 1.9.7 Sejam $P: C \rightarrow C$ uma transformação de primeiro retorno unicamente ergódica, e $v$ a única medida de probabilidade de Borel invariante por $P$. Seja $\varphi: \operatorname{dom}(P) \rightarrow \mathbb{R}$ uma função escada. Então as médias de Birkhoff $(1 / n) \sum_{k=0}^{n-1} \varphi\left(P^{k}(x)\right)\left(\right.$ resp. $\left.(1 / n) \sum_{k=0}^{n-1} \varphi\left(P^{-k}(x)\right)\right)$ convergem uniformemente em $\Omega_{+}$(resp. $\left.\Omega_{-}\right)$para $\int \varphi \mathrm{d} v$.

Demonstração: É uma consequência do Lema 1.9.6.

Proposição 1.9.8 Sejam $X \in \mathscr{H}, \gamma$ uma trajetória de $X$ satisfazendo $(a)-(c)$, C um círculo transversal especial passando por $\gamma$, e $P: C \rightarrow C$ a correspondente transformação de primeiro retorno. Então existem $N_{0} \in \mathbb{N}$, e funções escada $\varphi, \psi$ definidas em $\operatorname{dom}(P)$, satisfazendo $\varphi>\log |D P| e$ $\psi<\log \left|D P^{-1}\right|$, tais que:

(a) $\frac{1}{n} \sum_{k=0}^{n-1} \varphi\left(P^{k}(x)\right)<-\frac{c}{4}$, para todo $x \in \Omega_{+}$e $n \geq N_{0}$;

(b) $\frac{1}{n} \sum_{k=0}^{n-1} \psi\left(P^{-k}(x)\right)>\frac{c}{4}$, para todo $x \in \Omega_{-}$e $n \geq N_{0}$. 
Demonstração: Pela Proposição 1.9.1 e Teorema 1.8.2 existe uma função escada $\varphi$ definida em $\operatorname{dom}(P)$ tal que $\varphi>\log |D P| \mathrm{e}$

$$
\int \varphi \mathrm{d} v<-\frac{c}{2}
$$

Pela Proposição 1.9.7, existe $N_{1} \in \mathbb{N}$ tal que para todo $x \in \Omega_{+}$e para todo $n \geq N_{1}$,

$$
\frac{1}{n} \sum_{k=0}^{n-1} \varphi\left(P^{k}(x)\right)-\int \varphi \mathrm{d} v<\frac{c}{4}
$$

Assim, para todo $x \in \Omega_{+}$e para todo $n \geq N_{1}$,

$$
\frac{1}{n} \sum_{k=0}^{n-1} \varphi\left(P^{k}(x)\right)<-\frac{c}{4}
$$

Agora considere o campo vetorial $-X$ e a correspondente transformação de primeiro retorno $P^{-1}: C \rightarrow C$. É claro que

$$
\log \left|D P^{-1}\right|=-\log \left|D P \circ P^{-1}\right|
$$

Assim, como $P$ preserva a medida $v$, obtemos de (1.19) que:

$$
\int \log \left|D P^{-1}\right| \mathrm{d} v=-\int \log \left|D P \circ P^{-1}\right| \mathrm{d} v=-\int \log |D P| \mathrm{d} v>c
$$

Como o divergente $-X$ em cada de suas selas é maior ou igual a zero, temos pela Proposição 1.9.1 e pelo Teorema 1.8.2, e por (1.20) que existem funções escada $\psi$ definidas em $\operatorname{dom}(P)$ tais que $\psi<\log \left|D P^{-1}\right| \mathrm{e}$

$$
\int \psi \mathrm{d} v>\frac{c}{2}
$$

Pela Proposição 1.9.7, existe $N_{2} \in \mathbb{N}$ tal que para todo $x \in \Omega_{-}$e para todo $n \geq N_{2}$,

$$
\int \psi \mathrm{d} v-\frac{1}{n} \sum_{k=0}^{n-1} \psi\left(P^{-k}(x)\right)<\frac{c}{4}
$$

Assim, para todo $x \in \Omega_{-}$e para todo $n \geq N_{2}$,

$$
\frac{1}{n} \sum_{k=0}^{n-1} \psi\left(P^{-k}(x)\right)>\frac{c}{4}
$$

Para terminar a prova defina $N_{0}=\max \left\{N_{1}, N_{2}\right\}$.

Proposição 1.9.9 Sejam $X \in \mathscr{H}, \gamma$ uma trajetória de $X$ satisfazendo $(a)-(c), C$ um círculo trans- 
versal especial passando por $\gamma$, e $P: C \rightarrow C$ a correspondente transformação de primeiro retorno. Dados $0<\lambda<1$ e $\mu>1$, existe $N_{0} \in \mathbb{N}$ tal que

(a) $\left.P^{n}\right|_{\Omega_{+}}$é uma $\lambda$-contração infinitesimal para todo $n \geq N_{0}$;

(b) $\left.P^{-n}\right|_{\Omega_{-}}$é uma $\mu$-expansão infinitesimal para todo $n \geq N_{0}$.

Demonstração: A prova é uma consequência imediata da Proposições 1.9.4, 1.9.5 e 1.9.8.

Proposição 1.9.10 Sejam $X \in \mathscr{H}, \gamma$ uma trajetória de $X$ satisfazendo $(a)-(c), C$ um círculo transversal especial passando por $\gamma$ e $P: C \rightarrow C$ a correspondente transformação de primeiro retorno. Então:

(i) $\Omega_{+}$é todo o círculo transversal C menos um conjunto enumerável de pontos, cujas trajetórias positivas vão para selas de $X$;

(ii) Para cada $n \in \mathbb{N}$, $\operatorname{dom}\left(P^{n}\right)$ é todo o círculo transversal C menos um conjunto finito de pontos.

Demonstração: A mesma prova da Proposição 1.3.11 funciona neste caso.

Teorema C. A seguinte inclusão vale: $\mathscr{H} \subset \mathscr{C}$. Mais especificamente, se uma trajetória $\gamma$ tem as propriedades $(a)-(c)$, então ela também tem a propriedade $(\star)$.

Demonstração: Sejam $X \in \mathscr{H}, \gamma$ uma trajetória de $X$ tendo as propriedades $(a)-(c), C$ um círculo transversal especial passando por $\gamma$, e $P: C \rightarrow C$ a correspondente transformação de primeiro retorno. Primeiramente note que, pela Proposição 1.9.10, se $x \in \operatorname{dom}\left(P^{n}\right)$, então ou $x \in \Omega_{+}$ou $\omega\left(\gamma_{x}^{+}\right)$é uma sela de $X$. No primeiro caso, pela Proposição 1.9.9, dado $0<\lambda-\varepsilon<\lambda<1$ existe $N_{0} \in \mathbb{N}$ tal que para todo $x \in \Omega_{+}$e $n \geq N_{0}$,

$$
\left|D P^{n}(x)\right|<\lambda-\varepsilon
$$

Considere agora o conjunto $B=\operatorname{dom}\left(P^{n}\right) \backslash \Omega_{+}$. Note que $\omega\left(\gamma_{y}^{+}\right)$é uma sela para todo $y \in B$. Assim, como há somente um número finito de separatrizes de selas, temos que $B$ é um conjunto contável de maneira que $\Omega_{+}$é denso em $B$. Ainda, a aplicação:

$$
x \mapsto\left|D P^{n}(x)\right|=\left|D P\left(P^{n-1}(x)\right)\right| \cdots \cdot|D P(P(x))| \cdot|D P(x)|
$$


é contínua em $\operatorname{dom}\left(P^{n}\right)$. Assim para todo $x \in \operatorname{dom}\left(P^{n}\right)$ e $n \geq N_{0}$ :

$$
\left|D P^{n}(x)\right| \leq \lambda-\varepsilon<\lambda
$$

Logo $P^{n}$ é uma $\lambda$-contração infinitesimal em $C$. É claro que, usando transformações de Poincaré, podemos transportar este resultado para qualquer segmento transversal pequeno passando por $\gamma$. 


\title{
Estabilidade Assintótica no Infinito para
}

\author{
Campos Vetoriais Diferenciáveis
}

\subsection{Introdução}

Neste Capítulo provaremos o Teorema D enunciado na introdução. Para entender seu enunciado, faz-se necessário introduzir algumas definições.

Em todo este trabalho, assumimos que $\mathbb{R}^{2}$ está mergulhado na esfera de Riemann $\mathbb{R}^{2} \cup\{\infty\}$ e que "infinito" refere-se ao ponto no infinito $\infty$ de $\mathbb{R}^{2} \cup\{\infty\}$. Isto se aplica também a subespaços de $\mathbb{R}^{2} \cup\{\infty\}$ da forma $\mathbb{R} \backslash \bar{D}_{\sigma}$, onde $\sigma>0$ e $\bar{D}_{\sigma}=\left\{z \in \mathbb{R}^{2}:\|z\| \leq \sigma\right\}$. Dado um campo vetorial contínuo $X: \mathbb{R}^{2} \backslash \bar{D}_{\sigma} \rightarrow \mathbb{R}^{2}$ do plano, podemos estendê-lo ao campo vetorial $\widehat{X}:\left(\left(\mathbb{R}^{2} \backslash \bar{D}_{\sigma}\right) \cup\right.$ $\{\infty\}, \infty) \rightarrow\left(\mathbb{R}^{2}, 0\right)$ da esfera de Riemann que leva o $\infty$ no 0 . Note que permitimos que $\widehat{X}$ seja descontínuo no $\infty$. Daqui em diante, vamos identificar $X$ com sua extensão $\widehat{X}$.

Seja $X: \mathbb{R}^{2} \backslash \bar{D}_{\sigma} \rightarrow \mathbb{R}^{2}$ um campo vetorial contínuo. Dizemos que uma semitrajetória positiva (resp. negativa) $\gamma_{p}^{+}$(resp. $\gamma_{p}^{-}$) de $X$ vai para infinito (resp. vem do infinito) se $\omega\left(\gamma_{p}^{+}\right)=\infty$ (resp. $\left.\alpha\left(\gamma_{p}^{-}\right)=\infty\right)$. Seja $\left\{\Gamma_{n}\right\}_{1}^{\infty}$ uma seqüência de círculos topológicos. Dizemos que a seqüência $\left\{\Gamma_{n}\right\}_{1}^{\infty}$ tende ao infinito se para toda vizinhança $V$ de $\infty$, existe $N \in \mathbb{N}$ tal que $n \geq N$ implica que $\Gamma_{n} \subset V$.

Definição 2.1.1 Dizemos que $\infty$ é um atrator (resp. um repulsor) de um campo vetorial contínuo X se

(i) Existe uma sequência de círculos transversais a $X$ de classe $C^{1}$ tendendo ao infinito; 
(ii) Para algum $R \geq \sigma$, todas as semitrajetórias positivas (resp. negativas) de $X$ se iniciando em $p \in \mathbb{R}^{2} \backslash \bar{D}_{R}$ vão para o infinito (resp. vêm do infinito).

Alguns comentários são necessários para motivar a Definição 2.1.1. Primeiramente devemos observar que no caso $C^{1}$, a Definição 2.1.1 é equivalente a dizer que o campo vetorial $\widehat{X}$ induzido por $X$ na esfera de Riemann é localmente topologicamente equivalente em uma vizinhança do infinito ou a $p \mapsto-p$ ou a $p \mapsto p$ na origem, ver [2].

No caso contínuo ou diferenciável esta definição é insatisfatória porque não é possível falar aqui de equivalência topológica. Note que dizer que $\infty$ é um atrator ou repulsor de $X$ é mais forte que dizer que no exterior de um disco $\bar{D}_{R}$ todas as trajetórias vão para o infinito. Isto impedi que o infinito seja um atrator ou repulsor do campo vetorial constante, que apresenta setores elípticos no infinito, ver a Figura 2.1a. Além disso, a condição (i) da Definição 2.1.1 não poder ser enfraquecida. Com efeito, existem campos vetoriais que, apesar de admitirem um círculo transversal $\Gamma$ e satisfazerem (ii) da Definição 2.1.1, não admitem uma família de círculos transversais tendendo a infinito, ver a Figura 2.1b.

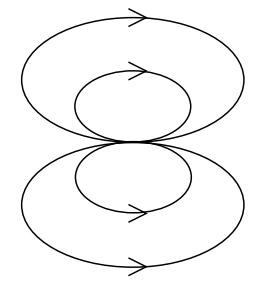

(a)

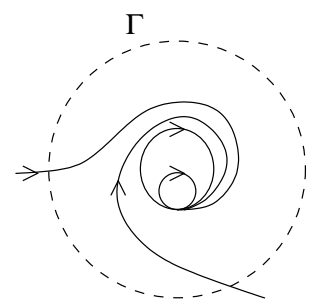

(b)

Figura 2.1: Dois campos vetoriais que não têm o ponto no infinito como um atrator

Seja $A$ um subconjunto Lebesgue mensurável de $\mathbb{R}^{n}$, e seja $f: A \rightarrow \mathbb{R}$ uma função mensurável. Definimos

$$
f^{+}(x)=\max \{f(x), 0\}, \quad f^{-}(x)=\max \{-f(x), 0\}
$$

e dizemos que $f: A \rightarrow \mathbb{R}$ é Lebesgue quase-integrável se

$$
\min \left\{\int_{A} f^{+} d \lambda, \int_{A} f^{-} d \lambda\right\}<\infty
$$

e neste caso definimos

$$
\int_{A} f d \lambda=\int_{A} f^{+} d \lambda-\int_{A} f^{-} d \lambda
$$

que é um número bem definido da reta real estendida $[-\infty, \infty]$.

Dado um campo vetorial diferenciável $X: U \subset \mathbb{R}^{2} \rightarrow \mathbb{R}^{2}$, denotamos por $\operatorname{Spec}(X)$ o conjunto de 
autovalores da derivada $D_{p} X$ de $X$ em $p$ quando $p$ percorre todo o conjunto $U$. Como usualmente, $\mathscr{R}(z)$ denote a parte real do número complexo $z$ e Traço $(D X): U \rightarrow \mathbb{R}$ refere-se à função que a cada ponto $p \in U$ assume o valor $\operatorname{Traço}\left(D_{p} X\right)$.

Agora seja

$\mathscr{D}(U)=\left\{X: U \rightarrow \mathbb{R}^{2}: X\right.$ é diferenciável e Traço $(D X)$ é Lebesgue quase-integrável em $\left.U\right\}$.

Definimos o índice de $X \in \mathscr{D}\left(\mathbb{R}^{2} \backslash \bar{D}_{\sigma}\right)$ no infinito como sendo o número da reta real estendida $[-\infty, \infty]$ definido por

$$
\mathscr{I}(X)=\int_{\mathbb{R}^{2}} \operatorname{Traço}(D \widehat{X}) d x \wedge d y
$$

onde $\widehat{X} \in \mathscr{D}\left(\mathbb{R}^{2}\right)$ é qualquer extensão globalmente injetiva diferenciável de $\left.X\right|_{\mathbb{R}^{2} \backslash D_{s}}$, para algum $s>\sigma$, o divergente da qual é Lebesgue quase-integrável em $\mathbb{R}^{2}$. Mostraremos (ver Corolário 2.2.12) que $\mathscr{I}(X)$ está bem definido. Podemos enunciar agora o Teorema principal deste Capítulo.

Teorema D. Seja $X: \mathbb{R}^{2} \backslash \bar{D}_{\sigma} \rightarrow \mathbb{R}^{2}$ um campo vetorial diferenciável (mas não necessariamente $\left.C^{1}\right)$. Se para algum $\varepsilon>0$, $\operatorname{Spec}(X)$ é disjunto de $(-\varepsilon, 0] \cup\{z \in \mathbb{C}: \mathscr{R}(z) \geq 0\}$, então

a) Para todo $p \in \mathbb{R}^{2} \backslash \bar{D}_{\sigma}$, há uma única semitrajetória positiva de $X$ se iniciando em $p$;

b) $\mathscr{I}(X)$, o índice de $X$ no infinito, é um número bem definido da reta real estendida $[-\infty, \infty)$;

c) Existe um vetor constante $v \in \mathbb{R}^{2}$ tal que se $\mathscr{I}(X)$ é $<0$ (resp. $\left.\geq 0\right)$, então o ponto no infinito da esfera de Riemann $\mathbb{R}^{2} \cup\{\infty\}$ é um repulsor (resp. um atrator) do campo vetorial $X+v$.

\subsection{Campos Vetoriais Diferenciáveis}

Seja $X: U \subset \mathbb{R}^{2} \rightarrow \mathbb{R}^{2}$ um campo vetorial contínuo definido em um conjunto aberto $U \subset \mathbb{R}^{2}$. Dizemos que uma curva curva $\gamma_{p}: I \rightarrow U$ de classe $C^{1}$ é uma solução do campo vetorial $X$ passando pelo ponto $p$ se $\gamma_{p}(0)=p$ e $\gamma_{p}^{\prime}(t)=X\left(\gamma_{p}(t)\right)$, para todo $t \in I$, onde $I \subset \mathbb{R}$ é um intervalo contendo zero. Pelo Teorema de Existência de Peano, por cada ponto $p \in U$, existe uma solução $\gamma_{p}: J\left(\gamma_{p}\right) \rightarrow$ $U$ definido em algum intervalo maximal aberto $J\left(\gamma_{p}\right)$ que depende tanto da solução $\gamma_{p}$ como do ponto de partida $p$. Por simplicidade, identificamos a solução $\gamma_{p}$ com sua imagem ao qual referimonos como a trajetória de $X$ passando por $p$ definida em $J\left(\gamma_{p}\right)$. Analogamente, $\gamma_{p}^{+}$(resp. $\gamma_{p}^{-}$) denotará a semitrajetória positiva (resp. negativa) de $X$ contida em $\gamma_{p}$ e se iniciando em $p$. Desta forma, $\gamma_{p}=\gamma_{p}^{-} \cup \gamma_{p}^{+}$. Dada uma semitrajetória $\gamma_{p}^{+}$positiva (resp. negativa), denotamos por $\omega\left(\gamma_{p}^{+}\right)$ (resp. $\alpha\left(\gamma_{p}^{-}\right)$) seu conjunto $\omega$-limite (resp. conjunto $\alpha$-limite). 
Dizemos que $p \in U$ é uma singularidade (resp. um ponto regular) de $X$ se $X(p)=0$ (resp. $X(p) \neq 0)$. Uma trajetória $\gamma$ é dita ser periódica se ela está definida em $\mathbb{R}$ e existe $\tau>0$ tal que $\gamma(t+\tau)=\gamma(t)$ para todo $t \in \mathbb{R}$. Relembramos que trajetórias de campos vetoriais contínuos podem se auto-interceptar ou interceptar-se mutuamente. Se uma trajetória se auto-intercepta então ela naturalmente contém uma trajetória periódica definida em $\mathbb{R}$. Se $U$ é simplesmente conexo então segue-se da Teoria de Índice que toda trajetória periódica de $X$ tem que conter uma singularidade no seu interior.

Dado um campo vetorial $X=(f, g)$, seja $X^{*}=(-g, f)$ o campo vetorial ortogonal a $X$. A mesma notação para intervalos de $\mathbb{R}$ será utilizada para arcos orientados de trajetória $[p, q],[p, q), \ldots$ (resp. $[p, q]^{*},[p, q)^{*}, \ldots$ ) de $X$ (resp. $X^{*}$ ), conectando os pontos $p$ e $q$. A orientação deste arcos é aquela induzida por $X$ (resp. $\left.X^{*}\right)$.

Definição 2.2.1 (Retângulo Compacto) Um retângulo compacto $R=R\left(p_{1}, p_{2} ; q_{1}, q_{2}\right) \subset U$ de um campo vetorial contínuo $X: U \subset \mathbb{R}^{2} \rightarrow \mathbb{R}^{2}$ é a região compacta cuja fronteira é formada por dois arcos de trajetória $\left[p_{1}, p_{2}\right],\left[q_{1}, q_{2}\right]$ de $X$ e dois arcos de $\left[p_{1}, q_{1}\right]^{*},\left[p_{2}, q_{2}\right]^{*}$ de $X^{*}$. Note que assumimos que o fluxo induzido por $X$ entra em $R$ por $\left[p_{1}, q_{1}\right]^{*}$ e deixa $R$ por $\left[p_{2}, q_{2}\right]^{*}$.

Dado um arco de trajetória $[p, q]^{*}$ of $X^{*}$, defina

$$
L\left([p, q]^{*}\right)=\left|\int_{[p, q]^{*}}\left\|X^{*}\right\| d s\right|
$$

onde $d s$ denota o elemento de comprimento de arco. Dado um arco de trajetória [p,q] (resp. $\left.[p, q]^{*}\right)$, definimos como $\ell([p, q])$ (resp. $\ell\left([p, q]^{*}\right)$ ) o comprimento de arco dele. A próxima fórmula é um corolário da Fórmula de Green tal qual apresentada em [53].

Lema 2.2.2 Seja $R=R\left(p_{1}, p_{2} ; q_{1}, q_{2}\right) \subset U$ um retângulo compacto de $X \in \mathscr{D}(U)$. Então

$$
L\left(\left[p_{2}, q_{2}\right]^{*}\right)-L\left(\left[p_{1}, q_{1}\right]^{*}\right)=\int_{R} \operatorname{Traço}(D X) d x \wedge d y
$$

O próximo resultado diz que um campo vetorial $X \in \mathscr{D}(U)$ cujo divergente é estritamente negativo em $U$ gera um semifluxo positivo.

Teorema 2.2.3 Seja $X \in \mathscr{D}(U)$ um campo vetorial sem singularidades tal que Traço $(D X)<0$ em $U$. Então para cada $p \in V$, há uma única semitrajetória positiva de $X$ passando por $p$. 
Demonstração: Assuma, por contradição, que existam duas semitrajetórias positivas $\gamma_{p}^{+}, \sigma_{p}^{+} \subset U$ iniciando-se em $p$. Então podemos tomar um triângulo (i.e. um retângulo degenerado) $R=R\left(p, q_{1} ; p, q_{2}\right) \subset U \operatorname{com}\left[p, q_{1}\right] \subset \gamma_{p}^{+} \mathrm{e}\left[p, q_{2}\right] \subset \sigma_{p}^{+}$. Pelo Lema 2.2.2,

$$
0<L\left(\left[q_{1}, q_{2}\right]^{*}\right)=\int_{R} \operatorname{Traço}(D X) d x \wedge d y<0
$$

que é uma contradição.

Lema 2.2.4 Seja $X \in \mathscr{D}(U)$ um campo vetorial tal que $\operatorname{Traço}(D X)<0$ em $U$. Assuma que $U$ não contenha nem singularidades nem trajetórias periódicas e que $K \subset U$ seja um conjunto compacto. Então não há semitrajetória positiva (resp. negativa) de X contida em $\mathrm{K}$.

Demonstração: No caso de uma semitrajetória positiva, a prova segue-se facilmente do Teorema 2.2.3 e do Teorema de Poincaré-Bendixson para semifluxos (ver [10]). No caso de uma semitrajetória negativa, iremos dar uma prova explícita baseada na negatividade do divergente de $X$. Assim assumimos que $\gamma^{-}$é uma semitrajetória negativa de $X$ contida num conjunto compacto $K \subset U$. Seja $p \in \alpha\left(\gamma^{-}\right)$e seja $\Sigma$ uma seção compacta ortogonal a $X$ passando por $p$. Sabe-se que nenhuma semitrajetória negativa pode se auto-interceptar, senão ela iria conter uma trajetória periódica. Assim $\gamma^{-}$intersecpta $\Sigma$ monotonamente e um número infinito de vezes. Denote por $\left\{p_{n}\right\}_{1}^{\infty}$ a sequência correspondente formada pelos pontos de intersecção, onde $p_{n} \rightarrow p$ conforme $n \rightarrow \infty$. Então, do Lema 2.2.2:

$$
L\left(\left[p_{j-1}, p_{j}\right]^{*}\right)-L\left(\left[p_{j}, p_{j+1}\right]^{*}\right)<0, \quad \forall j \in \mathbb{N}^{*},
$$

onde $\mathbb{N}^{*}=\mathbb{N} \backslash\{0\}$. Assim,

$$
L\left(\left[p_{0}, p_{1}\right]^{*}\right)-L\left(\left[p_{n}, p_{n+1}\right]^{*}\right)=\sum_{j=1}^{n} L\left(\left[p_{j-1}, p_{j}\right]^{*}\right)-L\left(\left[p_{j}, p_{j+1}\right]^{*}\right)<0, \quad \forall n \in \mathbb{N}^{*}
$$

Isto é,

$$
0<L\left(\left[p_{0}, p_{1}\right]^{*}\right)<L\left(\left[p_{n}, p_{n+1}\right]^{*}\right), \quad \forall n \in \mathbb{N}^{*}
$$

Mas isto é um absurdo pois $L\left(\left[p_{n}, p_{n+1}\right]^{*}\right) \rightarrow 0$ as $n \rightarrow \infty$. Desta forma, $\alpha\left(\gamma^{-}\right)=\emptyset$. Como $K$ é um compacto e $\gamma^{-} \subset K, \alpha\left(\gamma^{-}\right)$não pode ser vazio. Esta contradição encerra a prova.

Definição 2.2.5 Denotamos por $\mathscr{D}_{\sigma}$ o conjunto dos campos vetoriais diferenciáveis $X: \mathbb{R}^{2} \backslash \bar{D}_{\sigma} \rightarrow \mathbb{R}^{2}$ tais que $\operatorname{Spec}(X)$ é disjunto de $(-\varepsilon, 0] \cup\{z \in \mathbb{C}: \mathscr{R}(z) \geq 0\}$ para algum $\varepsilon>0$. 
Primeiramente deduzimos algumas propriedades úteis dos campos vetoriais na classe $\mathscr{D}_{\sigma}$. O próximo resultado mostra que se $X \in \mathscr{D}_{\sigma}$ então $\left.X\right|_{\mathbb{R}^{2} \backslash \bar{D}_{s}} \in \mathscr{D}\left(\mathbb{R}^{2} \backslash \bar{D}_{s}\right)$ para todo $s \geq \sigma$.

Lema 2.2.6 Seja $X \in \mathscr{D}_{\sigma}$ um campo vetorial diferenciável. Então para todo $s \geq \sigma$ temos que $\operatorname{Traço}(D X)<0$ em $\mathbb{R}^{2} \backslash \bar{D}_{s}$ e assim Traço $\left.(D X)\right|_{\mathbb{R}^{2} \backslash \bar{D}_{s}}: \mathbb{R}^{2} \backslash \bar{D}_{s} \rightarrow \mathbb{R}$ é Lebesgue quase-integrável.

Demonstração: Pelas hipóteses em $\operatorname{Spec}(X)$, para cada $p \in \mathbb{R}^{2} \backslash \bar{D}_{\sigma}$, todos os autovalores de $D_{p} X$ têm partes reais negativas de maneira que $\operatorname{Traço}(D X)<0$ em $\mathbb{R}^{2} \backslash \bar{D}_{s} \subset \mathbb{R}^{2} \backslash \bar{D}_{\sigma}$. A integrabilidade de Lebesgue de Traço $\left.(D X)\right|_{\mathbb{R}^{2} \backslash \bar{D}_{s}}: \mathbb{R}^{2} \backslash \bar{D}_{s} \rightarrow \mathbb{R}$ segue da definição.

Na prova do próximo Teorema fazemos uso do seguinte resultado de Gutierrez e Rabanal [26].

Teorema 2.2.7 Seja $X: \mathbb{R}^{2} \backslash \bar{D}_{\sigma} \rightarrow \mathbb{R}^{2}$ um campo vetorial diferenciável. Se para algum $\varepsilon>0$, $\operatorname{Spec}(X) \cap(-\varepsilon,+\infty)=\emptyset$, então existe $s_{0} \geq \sigma$ tal que $\left.X\right|_{\mathbb{R}^{2} \backslash \bar{D}_{s_{0}}}$ pode ser estendido a uma homeomorfismo local globalmente injetivo $\widetilde{X}: \mathbb{R}^{2} \rightarrow \mathbb{R}^{2}$.

Observação 2.2.8 Uma consequência imediata do Teorema 2.2.7 é que se $X \in \mathscr{D}_{\sigma}$ então fora de um disco grande $\bar{D}_{R} \supset \bar{D}_{\sigma}$, o campo vetorial X não tem singularidades. Em adição, pelo Lema 2.2.6, o divergente de X é negativo em $\mathbb{R}^{2} \backslash \bar{D}_{\sigma}$ de maneira que pelo Lema 2.2.2, X admite no máximo uma trajetória periódica contida em $\mathbb{R}^{2} \backslash \bar{D}_{\sigma}$. Assim podemos assumir $R$ grande o bastante para que $\mathbb{R}^{2} \backslash \bar{D}_{R}$ seja uma região livre de singularidades e órbitas periódicas. Colocado de outra maneira, $X$ não tem nem singularidades nem trajetórias periódicas no infinito. Como $\mathscr{D} \sigma$ é invariante por translação (i.e $X+v \in \mathscr{D}_{\sigma}$ sempre que $X \in \mathscr{D}_{\sigma}$ e $v \in \mathbb{R}^{2}$ ), temos que se $X \in \mathscr{D}_{\sigma}$ $e v \in \mathbb{R}^{2}$, então $X+v \in \mathscr{D}_{\sigma}$ e portanto não tem nem singularidades nem trajetórias periódicas no infinito.

Teorema 2.2.9 Seja $X \in \mathscr{D}_{\sigma}$ um campo vetorial diferenciável. Então para algum $s_{0} \geq \sigma$, existe $v \in \mathbb{R}^{2}$, c $>0$ e um homeomorfismo local globalmente injetivo $Y: \mathbb{R}^{2} \rightarrow \mathbb{R}^{2}$ tal que

1. $Y(0)=0$;

2. $\left.Y\right|_{\mathbb{R}^{2} \backslash \bar{D}_{s_{0}}}=\left.X\right|_{\mathbb{R}^{2} \backslash \bar{D}_{s_{0}}}+v$;

3. $\|Y(p)\|>$ c para qualquer $p \in \mathbb{R}^{2} \backslash \bar{D}_{s_{0}}$;

4. Traço $\left.(D Y)\right|_{\mathbb{R}^{2} \backslash \bar{D}_{s_{0}}}: \mathbb{R}^{2} \backslash \bar{D}_{s_{0}} \rightarrow \mathbb{R}_{-}$é Lebesgue quase-integrável; 
5. $\left.Y\right|_{\mathbb{R}^{2} \backslash \bar{D}_{s_{0}}}$ preserva orientação;

6. Y não tem nem singularidades nem trajetórias periódicas em $\mathbb{R}^{2} \backslash \bar{D}_{s_{0}}$;

7. $\left.Y\right|_{\mathbb{R}^{2} \backslash \bar{D}_{s_{0}}}$ gera um semifluxo positivo.

Demonstração: Pelas hipóteses sobre $\operatorname{Spec}(X)$, temos que $\operatorname{Spec}(X) \cap(-\varepsilon,+\infty)=\emptyset$. Então pelo Teorema 2.2.7 existem $s_{0} \geq \sigma$ e um homeomorfismo local globalmente injetivo $\widetilde{X}: \mathbb{R}^{2} \rightarrow \mathbb{R}^{2}$ que estende $\left.X\right|_{\mathbb{R}^{2} \backslash \bar{D}_{s_{0}}}$. Coloque $v=-\widetilde{X}(0)$ e $Y=\widetilde{X}+v$ para conseguir a aplicação desejada $Y$. (1) e (2) seguem imediatamente. (3) segue de (1) e do fato de a aplicação $Y$ ser globalmente injetiva e uma aplicação aberta. (4) segue de (2), da invariância de $\mathscr{D} \sigma$ por translações, e do Lema 2.2.6. Para provar (5), observe que $\operatorname{Det}\left(D_{p} Y\right)=\operatorname{Det}\left(D_{p} X\right)>0$ para todo $p \in \mathbb{R}^{2} \backslash \bar{D}_{s_{0}}$. (6) segue da Observação 2.2.8 sob a hipótese de que $s_{0}$ é grande o suficiente. Finalmente, (7) segue de (4), (6) e do Teorema 2.2.3.

Nas próximas seções, iremos explorar o Teorema 2.2.9 tão completamente quanto possível. Voltamo-nos agora para uma questão de teoria da medida. Para que $\mathscr{I}(X)$ esteja bem definida, temos que mostrar que existe alguma extensão global diferenciável de $\left.X\right|_{\mathbb{R}^{2} \backslash \bar{D}_{r}}$, para algum $r>\sigma$, cujo divergente é Lebesgue quase-integrável em $\mathbb{R}^{2}$. Isto é o propósito do próximo teorema. Note que a extensão contínua $\widetilde{X}: \mathbb{R}^{2} \rightarrow \mathbb{R}^{2}$ dada pelo Teorema 2.2 .7 pode não ser diferenciável em $\bar{D}_{s_{0}}$.

Teorema 2.2.10 Seja $X \in \mathscr{D}\left(\mathbb{R}^{2} \backslash \bar{D}_{\sigma}\right)$. Então, para algum $r>\sigma,\left.X\right|_{\mathbb{R}^{2} \backslash \bar{D}_{r}}$ admite uma extensão global diferenciável $\widetilde{X} \in \mathscr{D}\left(\mathbb{R}^{2}\right)$ cujo divergente é Lebes gue quase-integrável em $\mathbb{R}^{2}$.

Demonstração: Sejam $r_{1}>\sigma$ e $\lambda: \mathbb{R}^{2} \rightarrow[0,1]$ uma função bacia suave tal que $\lambda(z)=0$ para $\|z\| \leq r_{1}$ e $\lambda(z)=1$ para $\|z\| \geq r_{1}+1$. Dado $\varepsilon>0$, seja $X_{1}: \mathbb{R}^{2} \rightarrow \mathbb{R}^{2}$ uma aplicação $C^{1}$ tal que $\left\|X_{1}(z)-X(z)\right\|<\varepsilon$ para todo $r_{1} \leq\|z\| \leq r_{1}+1$. Defina $\widetilde{X}: \mathbb{R}^{2} \rightarrow \mathbb{R}^{2}$ como sendo uma aplicação diferenciável satisfazendo

$$
\widetilde{X}(z)=\lambda(z) X(z)+(1-\lambda(z)) X_{1}(z)
$$

onde como sempre definimos $\lambda(z) X(z)=0$ para $z \in \bar{D}_{\sigma}$.

$$
\begin{gathered}
\text { Seja } A=\bar{D}_{r_{1}} \text {, B }=\bar{D}_{r_{1}+1} \backslash D_{r_{1}} \text { e C }=\mathbb{R}^{2} \backslash D_{r_{1}+1} \text {. Temos que } \mathbb{R}^{2}=A \cup B \cup C \text {. Além disso, } \\
\left.\widetilde{X}\right|_{A}=\left.X_{1}\right|_{A}, \\
\left.\widetilde{X}\right|_{\mathrm{B}}=\left.\left.\lambda\right|_{\mathrm{B}} X\right|_{\mathrm{B}}+\left.\left(1-\left.\lambda\right|_{\mathrm{B}}\right) X_{1}\right|_{\mathrm{B}}, \\
\left.\widetilde{X}\right|_{\mathrm{C}}=\left.X\right|_{\mathrm{C}} .
\end{gathered}
$$


Como $X \in \mathscr{D}\left(\mathbb{R}^{2} \backslash \bar{D}_{\sigma}\right)$, temos que

$$
\min \left\{\int_{\mathbb{R}^{2} \backslash \bar{D}_{\sigma}} \operatorname{Traço}^{+}(D X) d x \wedge d y, \int_{\mathbb{R}^{2} \backslash \bar{D}_{\sigma}} \operatorname{Traço}^{-}(D X) d x \wedge d y\right\}<\infty
$$

Sem perda de generalidade, podemos assumir que

$$
\int_{\mathbb{R}^{2} \backslash \bar{D}_{\sigma}} \operatorname{Traço}^{+}(D X) d x \wedge d y<\infty
$$

Da suavidade de $X_{1}$ e de (2.1), obtemos que $\int_{A} \operatorname{Traço}^{+}(D \widetilde{X}) d x \wedge d y<\infty$. Por outro lado, de (2.3) e (2.4), $\int_{C} \operatorname{Traço}^{+}(D \widetilde{X}) d x \wedge d y \leq \int_{\mathbb{R}^{2} \backslash \bar{D}_{\sigma}} \operatorname{Traço}^{+}(D X) d x \wedge d y<\infty$. A prova estará terminada se mostrarmos que $\int_{B} \operatorname{Traço}^{+}(D \widetilde{X}) d x \wedge d y<\infty$. Diferenciando a equação (2.2) obtemos para $z \in B$,

$$
\begin{aligned}
\operatorname{Traço}\left(D_{z} \widetilde{X}\right)= & \lambda(z) \operatorname{Traço}\left(D_{z} X\right)+(1-\lambda(z)) \operatorname{Traço}\left(D_{z} X_{1}\right)+ \\
& +\lambda_{x}(z)\left(f(z)-f_{1}(z)\right)+\lambda_{y}(z)\left(g(z)-g_{1}(z)\right),
\end{aligned}
$$

onde $X=(f, g)$ e $X_{1}=\left(f_{1}, g_{1}\right)$. Como $\left\|X_{1}-X\right\|<\varepsilon$ em $B$, temos que $f_{1}(z)-f(z)$ e $g(z)-$ $g_{1}(z)$ são limitadas em $B$. A função $\lambda$ e suas derivadas parciais são também limitadas. Além disso, Traço $\left(D_{z} X_{1}\right)$ é uma função suave em um compacto $B$. Finalmente, de (2.4) segue-se que $\int_{B} \operatorname{Traço}^{+}(D X) d x \wedge d y<\infty$. De (2.5) obtemos que $\int_{B} \operatorname{Traço}^{+}(D \widetilde{X}) d x \wedge d y<\infty$. Assim, do que foi feito acima e usando que $\mathbb{R}^{2}=A \cup B \cup C$, segue-se que $\int_{\mathbb{R}^{2}} \operatorname{Traço}^{+}(D \widetilde{X}) d x \wedge d y<\infty$ de modo que $\operatorname{Traço}(D \widetilde{X})$ é Lebesgue quase-integrável. Para terminar a prova assuma $r=r_{1}+1$ e use (2.3).

Vamos precisar do seguinte Lema.

Lema 2.2.11 Seja $X \in \mathscr{D}\left(\mathbb{R}^{2} \backslash \bar{D}_{\sigma}\right)$ e $\widehat{X_{1}}, \widehat{X_{2}} \in \mathscr{D}\left(\mathbb{R}^{2}\right)$ extensões globais diferenciáveis de $\left.X\right|_{\mathbb{R}^{2} \backslash \bar{D}_{r}}$ para algum $r>\sigma$, isto é, $\widehat{X}_{i}(z)=X(z)$, para todo $z$ com $\|z\|>r$ e para $i=1,2$. Então

$$
\int_{\mathbb{R}^{2}} \operatorname{Traço}\left(D \widehat{X}_{1}\right) d x \wedge d y=\int_{\mathbb{R}^{2}} \operatorname{Traço}\left(D \widehat{X}_{2}\right) d x \wedge d y
$$

Demonstração: Devido à Fórmula de Green tal qual apresentada em [53], a prova da Proposição 2.1 de [2] (que é a versão $C^{1}$ do Lema 2.2.11) também funciona neste caso.

Corolário 2.2.12 Seja $X \in \mathscr{D}_{\sigma}$ um campo vetorial diferenciável. Então o índice $\mathscr{I}(X)$ de $X$ no infinito é um número bem definido da reta real estendida $[-\infty, \infty)$. 
Demonstração: Segue-se do Lema 2.2.6 e do Teorema 2.2.10 que, para algum $r>\sigma,\left.X\right|_{\mathbb{R}^{2} \backslash \bar{D}_{r}}$ admite uma extensão global diferenciável $\widehat{X} \in \mathscr{D}\left(\mathbb{R}^{2}\right)$ cujo divergente é Lebesgue quase-integrável em $\mathbb{R}^{2}$. Do Lema 2.2.11, $\mathscr{I}(X)$ não depende da extensão de maneira que ela está bem definida. Como no infinito Traço $(D X)$ é negativo, temos que $\mathscr{I}(X)<\infty$.

\subsection{Seções Transversais a Campos Vetoriais Contínuos}

Quando construindo seções transversais contínuas a campos vetoriais suaves podemos usufruir de várias ferramentas tais como a dependência contínua do fluxos com relação às condições iniciais e o Teorema do Fluxo Tubular. No caso contínuo, o panorama resulta ser diferente porque a unicidade local de soluções falha. Apesar disso, como o seguinte resultado nos mostra, ainda temos algum tipo de dependência contínua com relação às condições iniciais.

Primeiramente introduzimos um pouco de notação. Seja $F: V \subset \mathbb{R}^{n} \rightarrow \mathbb{R}^{n}$ um campo vetorial contínuo. Se $\gamma_{p}$ é uma trajetória de $F$ passando por $p \in V$, então $J\left(\gamma_{p}\right)$ denota seu intervalo maximal de existência. denotamos por $J(p)$ o subconjunto da reta real

$$
J(p)=\bigcap_{\gamma_{p}}\left\{J\left(\gamma_{p}\right): \gamma_{p} \text { é uma trajetória de } F \text { passando por } p\right\}
$$

que, pelo Teorema de Existência de Peano, é um intervalo contendo $p$ (ver [61, Corollary 4]).

Lema 2.3.1 Seja $F: V \subset \mathbb{R}^{n} \rightarrow \mathbb{R}^{n}$ um campo vetorial contínuo definido em um conjunto aberto $V$. Seja $p_{0} \in V$ e assuma que $J\left(p_{0}\right) \supset[0, \tau]$. Então para cada $\varepsilon>0$, existe $\delta>0$, tal que se $\left\|p-p_{0}\right\|<\delta$ então

(i) $J(p) \supset[0, \tau]$

(ii) Para cada trajetória $\gamma_{p}$ de $F$ passando por $p$, existe alguma trajetória $\gamma_{p_{0}}$ de $F$ passando por $p_{0}$ tal que $\left\|\gamma_{p}(t)-\gamma_{p_{0}}(t)\right\|<\varepsilon$ para todo $t \in[0, \tau]$.

Demonstração: Ver [61, Teorema 4] (ver também [5]).

Nos próximos teoremas assumimos que as semitrajetórias positivas de $X$ são únicas e assim que $X$ gera um semifluxo positivo. 
Teorema 2.3.2 Seja $X: U \subset \mathbb{R}^{2} \rightarrow \mathbb{R}^{2}$ um campo vetorial contínuo com semitrajetórias positivas únicas, definidas em um conjunto aberto $U$ sem singularidades; $\gamma$ uma semitrajetória positiva de $X$ com intervalo maximal de existência $J(\gamma) \supset[0, \tau], z_{1}=\gamma(0)$ e $z_{2}=\gamma(\tau)$; e seja $\Sigma_{2}$ uma seção local transvesal a $X$ passando por $z_{2}$. Então, em cada componente conexa de $\Sigma_{2} \backslash\left\{z_{2}\right\}$, existe um ponto $\widetilde{z}_{2}$ arbitrariamente próximo a $z_{2}$, e um segmento $C^{1} \Delta$ transversal a $X$, iniciando-se em $z_{1}$, terminando em $\widetilde{z}_{2}$, e próximo ao subarco de trajetória $\left[z_{1}, z_{2}\right] \subset \gamma$ de $X$.

Demonstração: Como $J(\gamma)$ é aberto, podemos escolher $\bar{\tau}>\tau$ em $J(\gamma)$. Seja $\widetilde{X}: U \subset \mathbb{R}^{2} \rightarrow \mathbb{R}^{2}$ um campo vetorial transversal a $X$. Desejamos encontrar um segmento transversal a $X$ que, para algum $\lambda>0$, é uma trajetória do campo vetorial perturbado $X_{\lambda}: U \subset \mathbb{R}^{2} \rightarrow \mathbb{R}^{2}$ definido por $X_{\lambda}=X+\lambda \widetilde{X}$. Para tanto, expandimos o espaço de fases de modo que ele inclua o parâmetro $\lambda$. Para tal fim, consideramos o campo vetorial estendido $F: U \times[0,1] \rightarrow \mathbb{R}^{3}$ definido por $F(z, \lambda)=\left(X_{\lambda}(z), 0\right)$. Seja $\pi_{1}: \mathbb{R}^{2} \times \mathbb{R} \rightarrow \mathbb{R}^{2}$ e $\pi_{2}: \mathbb{R}^{2} \times \mathbb{R} \rightarrow \mathbb{R}$ as projeções canônicas. É claro que se $\gamma_{(z, \lambda)}$ é uma trajetória de $F$ passando por $(z, \lambda) \in U \times[0,1]$, então $\pi_{1} \circ \gamma_{(z, \lambda)}$ é uma trajetória de $X_{\lambda}$ passando por $z$ e $\left(\pi_{2} \circ \gamma_{(z, \lambda)}\right)(t) \equiv \lambda$. Em particular, como $X_{0}=X$ gera um semifluxo positivo, todas semitrajetórias positivas de $F$ passando por $(z, 0) \in U \times[0,1]$ são únicas. Assim a única semitrajetória positiva de $F$ passando por $\left(z_{1}, 0\right)$ é $\gamma_{\left(z_{1}, 0\right)}(t)=(\gamma(t), 0)$. Desta forma, $J\left(z_{1}, 0\right)=J(\gamma) \supset[0, \bar{\tau}]$. Segue-se do Lema 2.3.1 que dado $\varepsilon>0$, existe $\delta>0$ tal que se $\left\|\left(z_{1}, \lambda\right)-\left(z_{1}, 0\right)\right\|<\delta$ então toda trajetória $\gamma_{\left(z_{1}, \lambda\right)}$ de $F$ passando por $\left(z_{1}, \lambda\right)$ satisfaz $J\left(\gamma_{\left(z_{1}, \lambda\right)}\right) \supset[0, \bar{\tau}]$ e $\left\|\gamma_{\left(z_{1}, \lambda\right)}(t)-(\gamma(t), 0)\right\|<\varepsilon, \forall t \in[0, \bar{\tau}]$. Para cada $\left(z_{1}, \lambda\right) \in U \times[0,1]$, escolha alguma trajetória $\gamma_{\left(z_{1}, \lambda\right)}$ de $F$ se iniciando em $\left(z_{1}, \lambda\right)$ e coloque $\gamma_{\lambda}=\pi_{1} \circ \gamma_{\left(z_{1}, \lambda\right)}$. Assim $\gamma_{\lambda}$ é uma trajetória de $X_{\lambda}$ iniciando-se em $z_{1}$. Pelo que foi feito acima, se $\lambda$ é suficientemente pequeno, então $J\left(\gamma_{\lambda}\right) \supset[0, \bar{\tau}]$ e $\sup _{t \in[0, \bar{\tau}]}\left\|\gamma_{\lambda}(t)-\gamma(t)\right\|<\varepsilon$. Portanto, como $\gamma$ cruza $\Sigma_{2}$ transversalmente em $z_{2}=\gamma(\tau)$, temos que existe $\tau_{2} \in[0, \bar{\tau}]$ tal que $\gamma_{\lambda}\left(\tau_{2}\right) \in \Sigma_{2}$. Coloque $\widetilde{z}_{2}(\lambda)=\gamma_{\lambda}\left(\tau_{2}\right)$ e seja $\Delta(\lambda)=\left[z_{1}, \widetilde{z}_{2}(\lambda)\right] \subset \gamma_{\lambda}$ o subarco de trajetória de $\gamma_{\lambda}$ conectando $z_{1}$ a $\widetilde{z}_{2}(\lambda)$. É fácil ver que se $\lambda>0$ é pequeno o bastante então $\widetilde{z}_{2}=\widetilde{z}_{2}(\lambda)$ e o segmento $\Delta=\Delta(\lambda)$ tem todas as propriedades requeridas. Para conseguir um ponto $\widetilde{z}_{2}$ na outra componente conexa de $\Sigma_{2} \backslash\left\{z_{2}\right\}$, substitua $\widetilde{X}$ por $-\widetilde{X}$ e proceda da mesma maneira.

\subsection{Pseudo Setor Hiperbólico no Infinito}

Definição 2.4.1 (Pseudo Setor Hiperbólico) Seja $X \in \mathscr{D}_{\sigma}$ e $S=S\left(p_{1}, p_{2} ; q_{1}, q_{2},\left\{\sigma_{i}\right\}\right) \subset \mathbb{R}^{2} \backslash \bar{D}_{\sigma} a$ região ilimitada cuja fronteira $\partial S$ é constituída por duas semitrajetórias ilimitadas $\left[q_{1}, \infty\right) e\left(\infty, q_{2}\right]$ de $X$, um arco compacto de trajetória $\left[p_{1}, p_{2}\right]$ de $X$, dois arcos de trajetória $\left[p_{1}, q_{1}\right]^{*},\left[p_{2}, q_{2}\right]^{*}$ de $X^{*}$, e um conjunto no máximo enumerável (que poder ser vazio) de trajetórias duas a duas disjuntas 


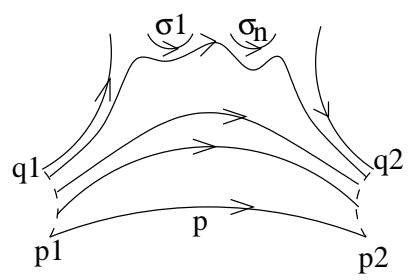

Figura 2.2: Pseudo Setor Hiperbólico

$\sigma_{1}, \sigma_{2}, \cdots, \sigma_{n}, \cdots$ que se iniciam e terminam no $\infty$ (ver Figure 2). Relembramos que tal região é um pseudo setor hiperbólico de X se as seguintes condições são satisfeitas

(1) Para cada $z \in\left[p_{1}, q_{1}\right)^{*}$, existe um arco de trajetória $[z, \pi(z)] \subset S$ de $X$ iniciando-se em $z \in\left[p_{1}, q_{1}\right)^{*}$ e terminando em $\pi(z) \in\left[p_{2}, q_{2}\right]^{*} ;$

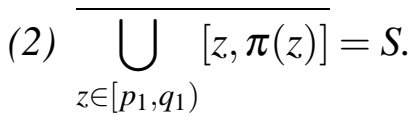

Desta maneira, a aplicação $\pi:\left[p_{1}, q_{1}\right)^{*} \rightarrow\left[p_{2}, q_{2}\right]^{*}$ é nada menos que a Aplicação de Poincaré induzida pelo semifluxo positivo associado com $\left.X\right|_{\mathbb{R}^{2} \backslash \bar{D}_{\sigma}}$ (ver Lema 2.2.6 e Teorema 2.2.3). Vamos chamar a parte ilimitada de $\partial S$ o conjunto

$$
\partial_{+} S=\left[q_{1}, \infty\right) \cup\left(\infty, q_{2}\right] \cup \bigcup_{i=1}^{\infty} \sigma_{i} \subset \partial S
$$

Daqui em diante todos os esforços serão concentrados na direção de provar o teorema principal desta seção, enunciado abaixo. No que segue, o campo vetorial $Y$ e a constante positiva $s_{0}$ são como no Teorema 2.2.9.

Teorema 2.4.2 Não há nenhum pseudo setor hiperbólico de $Y$ contido em $\mathbb{R}^{2} \backslash \bar{D}_{s}$, para qualquer $s \geq s_{0}$.

Antes de provar o Teorema 2.4.2, damos alguns lemas preparatórios.

Lema 2.4.3 Sejam $s \geq s_{0} e\left[p_{1}, q_{1}\right]^{*} \in \mathbb{R}^{2} \backslash \bar{D}_{s}$ um arco de trajetória de $Y^{*}$ fixado. Então, existe um $K>0$ tal que para qualquer retângulo compacto $R=R\left(p_{1}, p ;, r_{1}, r\right) \subset \mathbb{R}^{2} \backslash \bar{D}_{s}$ de $Y$ satisfazendo $\left[p_{1}, r_{1}\right]^{*} \subset\left[p_{1}, q_{1}\right]^{*}$ temos que $\ell\left([p, r]^{*}\right)<K$. Ver a Figura 3. 
Demonstração: Do Lema 2.2.2 segue-se que para qualquer retângulo $R\left(p_{1}, p ;, r_{1}, r\right) \subset \mathbb{R}^{2} \backslash \bar{D}_{s}$,

$$
L\left([p, r]^{*}\right)-L\left(\left[p_{1}, r_{1}\right]^{*}\right)=\int_{R} \operatorname{Traço}(D Y) d x \wedge d y<0
$$

Definindo $d=\sup \left\{\|Y(z)\|: z \in\left[p_{1}, q_{1}\right]^{*}\right\}$ e usando (3) do Teorema 2.2.9 resulta

$$
c \ell\left([p, r]^{*}\right) \leq\left|\int_{[p, r]^{*}}\|Y\| d s\right|=L\left([p, r]^{*}\right)<L\left(\left[p_{1}, r_{1}\right]^{*}\right)=\left|\int_{\left[p_{1}, r_{1}\right]^{*}}\|Y\| d s\right| \leq d \ell\left(\left[p_{1}, r_{1}\right]^{*}\right) .
$$

Portanto, definindo $K=\frac{d}{c} \ell\left(\left[p_{1}, q_{1}\right]^{*}\right)$, obtemos

$$
\ell\left([p, r]^{*}\right) \leq \frac{d}{c} \ell\left(\left[p_{1}, r_{1}\right]^{*}\right) \leq \frac{d}{c} \ell\left(\left[p_{1}, q_{1}\right]^{*}\right)=K
$$

Lema 2.4.4 Seja $S=S\left(p_{1}, p_{2} ; q_{1}, q_{2},\left\{\sigma_{i}\right\}\right)$ um pseudo setor hiperbólico de $Y$ contido em $\mathbb{R}^{2} \backslash \bar{D}_{s}$ para algum $s \geq s_{0}$. Então para cada $q \in \partial_{+} S$, existe $p \in\left[p_{1}, p_{2}\right]$ e arco de trajetória $[p, q]^{*} \subset S$ de $Y^{*}$ partindo de $p$ e terminando em $q$.

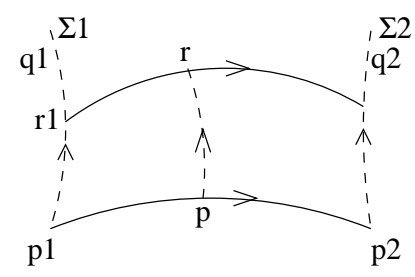

Figura 2.3:

Demonstração: Sejam $q \in \partial_{+} S$ e $\pi:\left[p_{1}, q_{1}\right)^{*} \rightarrow\left[p_{2}, q_{2}\right]^{*}$ a aplicação de Poincaré induzida pelo semifluxo positivo gerado por $\left.Y\right|_{\mathbb{R}^{2} \backslash \bar{D}_{s_{0}}}$. Seja $\left\{z_{n}\right\}_{1}^{\infty} \rightarrow q_{1}$ uma sequência em $\left[p_{1}, q_{1}\right]^{*}$. Coloque $w_{n}=\pi\left(z_{n}\right) \in\left[p_{2}, q_{2}\right]^{*}$. Então $w_{n} \rightarrow q_{2}$ conforme $n \rightarrow \infty$ e o arco de trajetória $\left[z_{n}, w_{n}\right]$ de $Y$ se acumula em $\partial_{+} S$. Seja $\gamma_{q}^{-}$qualquer semitrajetória negativa de $Y^{*}$ se iniciando em $q$. Assim, para algum $n \in \mathbb{N}, \gamma_{q}^{-}$vai para o retângulo compacto $R\left(p_{1}, p_{2}, z_{n}, w_{n}\right)$. Agora, pelo Lema 2.2.4, $\gamma_{q}^{-}$ intercepta $\left[p_{1}, p_{2}\right] \cup\left[p_{1}, z_{n}\right]^{*} \cup\left[p_{2}, w_{n}\right]^{*}$ e assim $\gamma_{q}^{-}$intercepta $A=\left[p_{1}, p_{2}\right] \cup\left[p_{1}, q_{1}\right]^{*} \cup\left[p_{2}, q_{2}\right]^{*}$. Através de um procedimento de colagem de arcos, como descrito acima, podemos encontrar um arco de trajetória $[p, q]^{*}$ de $Y^{*}$ como requerido pelo lema. De fato, se $\gamma_{q}^{-}$encontra $A$, pela primeira vez, em $p \in\left[p_{1}, p_{2}\right]$, então o subarco $[p, q]$ de $\gamma_{q}^{-}$satisfaz as condições requeridas neste lema; se $\gamma_{q}^{-}$intercepta $A$, pela primeira vez, em $r \in\left[p_{1}, q_{1}\right]^{*}$ (resp. em $r \in\left[p_{2}, q_{2}\right] *$ ), o arco $[p, q]$ formado 
pela união de subarco $\left[p_{1}, r\right]^{*}$ de $\left[p_{1}, q_{1}\right]^{*}$ (resp. $\left[p_{2}, r\right]^{*}$ de $\left[p_{2}, q_{2}\right] *$ ) como o subarco $[r, q]^{*}$ de $\gamma_{q}^{-}$ satisfaz as condições requeridas neste lema.

Lema 2.4.5 Sejam $s \geq s_{0}$ e $S=S\left(p_{1}, p_{2} ; q_{1}, q_{2},\left\{\sigma_{i}\right\}\right) \subset \mathbb{R}^{2} \backslash \bar{D}_{s}$ um pseudo setor hiperbólico de $Y$. Então existe constante $K>0$ tal que qualquer arco de trajetória $\gamma^{*}=[p, q]^{*} \subset S$ de $Y^{*}$ conectando um ponto $p \in\left[p_{1}, p_{2}\right]$ com um ponto $q \in \partial S$ satisfaz $\ell\left(\gamma^{*}\right) \leq K$.

Demonstração: Como $\gamma^{*}=[p, q]^{*}$ termina em $q \in \partial S$, então ou $\gamma^{*}$ termina em $\left[p_{1}, q_{1}\right]^{*} \cup\left[p_{2}, q_{2}\right]^{*}$, ou ele termina em $\partial_{+} S$. Por um procedimento de colagem de arcos, como decrito na prova do Lema 2.4.4, podemos assumir que $q \in \partial_{+} S$. Seja $\left\{r_{1}^{(n)}\right\}_{1}^{\infty} \rightarrow q_{1}$ uma sequência em $\left[p_{1}, q_{1}\right]^{*}$. Denote por $\gamma_{n}$ a semitrajetória positiva de $\left.Y\right|_{R^{2} \backslash \bar{D}_{s}}$ iniciando-se em $r_{1}^{(n)}$, cuja unicidade segue de (7) do Teorema 2.2.9. Coloque $r^{(n)}=\gamma_{n} \cap \gamma^{*}$. Ver Figura 4. Como $\gamma_{n}$ se acumula em $\partial_{+} S$ conforme $n$ tende ao infinito, temos que $\gamma^{*}=\limsup \left[p, r^{(n)}\right]^{*}$. Então, do Lema 2.4.3, existe constante $K>0$, não dependendo de $\gamma^{*}$, tal que $\ell\left(\gamma^{*}\right)=\lim _{n \rightarrow \infty} \ell\left(\left[p, r^{(n)}\right]^{*}\right) \leq K$.

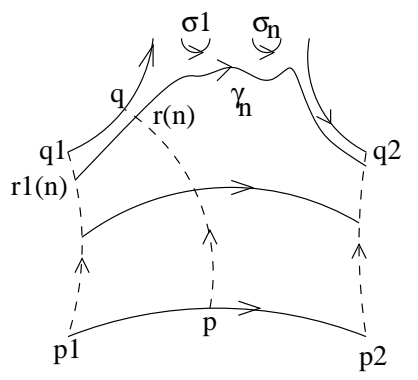

Figura 2.4:

Lema 2.4.6 Seja $s \geq s_{0}$ e seja $S=S\left(p_{1}, p_{2} ; q_{1}, q_{2},\left\{\sigma_{i}\right\}\right) \subset \mathbb{R}^{2} \backslash \bar{D}_{s}$ um pseudo setor hiperbólico de $Y$. Então há uma constante $K>0$ tal que $d\left(q,\left[p_{1}, p_{2}\right]\right) \leq K$, para todo $q \in \partial_{+} S$.

Demonstração: Seja $q \in \partial_{+} S$. Dos Lemas 2.4 .4 e 2.4.5, existe constante $K>0$ não dependendo de $q$, e um arco de trajetória $[p, q]^{*} \subset S$ de $Y^{*} \operatorname{com} p \in\left[p_{1}, p_{2}\right]$ and $\ell\left([p, q]^{*}\right) \leq K$. Assim $d\left(q,\left[p_{1}, p_{2}\right]\right) \leq K$, para todo $q \in \partial_{+} S$.

Demonstração: (do Teorema 2.4.2). Assuma, por contradição, que $Y$ admita um pseudo setor hiperbólico $S=S\left(p_{1}, p_{2} ; q_{1}, q_{2},\left\{\sigma_{i}\right\}\right)$ contido em $\mathbb{R}^{2} \backslash \bar{D}_{s}$ para algum $s \geq s_{0}$. Pelo Lema 2.4.6, existe constante $K>0$ tal que $d\left(q,\left[p_{1}, p_{2}\right]\right) \leq K$, para todo $q \in \partial_{+} S$. Em particular, como $\left[p_{1}, p_{2}\right]$ é compacto, temos que $\partial_{+} S$ é um conjunto limitado. Isto é um absurdo. 


\subsection{Círculos Transversais ao Redor do Infinito}

Esta seção é devotada à construção de um círculo $C^{1}$, contido em $\mathbb{R}^{2} \backslash \bar{D}_{s}$, transversal ao campo vetorial diferenciável $Y$, para $s$ arbitrariamente grande. Denote por $\mathscr{C}=\mathscr{C}_{s}$ a classe de círculos $C^{1}$ por partes contidos em $\mathbb{R}^{2} \backslash \bar{D}_{s}$. Um círculo $C \in \mathscr{C}$ é dito ser internamente (resp. externamente) tangente a um campo vetorial diferenciável $X: \mathbb{R}^{2} \backslash \bar{D}_{s} \rightarrow \mathbb{R}^{2}$ em $p \in C$ se para cada trajetória $\gamma$ passando por $p$, existe $\varepsilon>0$ tal que $\gamma(t) \in D(C)$ (resp. $\gamma(t) \in \mathbb{R}^{2} \backslash \bar{D}(C)$ ) para todo $0<|t|<\varepsilon$, onde $D(C)$ (resp. $\bar{D}(C)$ ) denota o disco aberto (resp. compacto) limitado por $C$. Se este é o caso, dizemos que $C$ tem tangência interna (resp. externa) $\operatorname{com} X$ em $p$. Um círculo $C \in \mathscr{C}$ é dito estar em posição geral com o campo vetorial diferenciável $X: \mathbb{R}^{2} \backslash \bar{D}_{s} \rightarrow \mathbb{R}^{2}$ se existir um subconjunto $F$ de $C$ no máximo finito tal que (i) $X$ é transversal a $C$ em $C \backslash F$; (ii) $C$ é internamente ou externamente tangente a $X$ em cada ponto de $F$; (iii) Qualquer trajetória de $X$ encontra $C$ tangencialmente em no máximo um ponto. Denotamos a classe de círculos em $\mathbb{R}^{2} \backslash \bar{D}_{s}$ em posição geram com $X$ por $\mathscr{G} \mathscr{P}(X, s)$. No que segue, $Y$ é o campo vetorial do Teorema 2.2.9.

Lema 2.5.1 Para cada $s \geq s_{0}, \mathscr{G} \mathscr{P}(Y, s) \neq \emptyset$.

Demonstração: Seja $C=\left\{p \in \mathbb{R}^{2}:\|p\|=s+1\right\}$ e seja $0<\varepsilon<0.1$. Por (4) do Teorema 2.2.9, $p \mapsto \frac{Y(p)}{\|Y(p)\|}$ é uma aplicação contínua definida em $\mathbb{R}^{2} \backslash \bar{D}_{s} \subset \mathbb{R}^{2} \backslash \bar{D}_{s_{0}}$. Assim existe uma cobertura $\{B i\}_{i=1}^{N}$ de $C$ por bolas abertas contidas em $\mathbb{R}^{2} \backslash \bar{D}_{s}$ tão pequenas que

(a) se $p, q$ pertencem à mesma bola $B_{i}$ então $\left\|\frac{Y(p)}{\|Y(p)\|}-\frac{Y(q)}{\|Y(q)\|}\right\|<\varepsilon$.

Seja $m>0$ um número natural tão grande que $\frac{8(s+1)}{m}$ é um número de Lebesgue para a cobertura acima. Para todo $j \in\{0,1,2, \ldots, m\}$, seja $p_{j}=(s+1)\left(\cos \frac{2 \pi j}{m}, \sin \frac{2 \pi j}{m}\right) \in C$. Desta maneira, para todo $j \in\{0,1,2, \cdots, m-1\},\left\|p_{j+1}-p_{j}\right\|<\frac{2 \pi(s+1)}{m}<\frac{8(s+1)}{m}$. Para todo $j \in\{0,1,2, \ldots, m-1\}$, selecione $q_{j} \in \mathbb{R}^{2}$ de maneira que $\Delta_{j}=\left\{p_{j}, p_{j+1}, q_{j}\right\}$ consiste dos vértices de um triângulo equilátero; certamente, o diâmetro de $\Delta_{j}$ é menor que o número de Lebesgue $\frac{8(s+1)}{m}$ e assim $\Delta_{j} \subset \mathbb{R}^{2} \backslash \bar{D}_{s}$, para todo $j$. Se o arco $\left[p_{j}, p_{j+1}\right]_{C} \subset C$ é transversal a $Y$, defina $\Gamma_{j}=\left[p_{j}, p_{j+1}\right]_{C}$; de outra maneira, defina $\Gamma_{j}$ como a união dos segmentos de retas $\left[p_{j}, q_{j}\right]$ e $\left[q_{j}, p_{j+1}\right]$. Tome $m$ suficientemente grande, digamos $m>16$, de modo que a variação angular do vetor tangente unitário a $C$ dentro de $\left[p_{j}, p_{j+1}\right]_{C}$ é menor que $\frac{\pi}{8}$ para todo $j \in\{0,1,2, \cdots, m-1\}$. Disto e de $(a)$ segue-se que $\Gamma_{j} \backslash \Delta_{j}$ é transversal a $Y$. O círculo $\Gamma=\cup_{j=0}^{m-1} \Gamma_{j}$ é transversal a $Y$ exceto possivelmente em um subconjunto finito $\cup_{j=0}^{m-1} \Delta_{j}$. Como $\left.Y\right|_{\mathbb{R}^{2} \backslash \bar{D}_{s}}$ não tem singularidades nem trajetórias periódicas, pelo Teorema de Poincaré-Bendixson para semifluxos (ver [10]) nenhuma semitrajetória positiva de $\left.Y\right|_{\mathbb{R}^{2} \backslash \bar{D}_{s}}$ é recorrente. 
Não é difícil concluir disto que $\Gamma$ pode ser aproximada por um círculo $C^{1}$ por partes de $\mathscr{G} \mathscr{P}(Y, s)$.

Observação 2.5.2 Sejam $s \geq s_{0} e C \in \mathscr{G} \mathscr{P}(Y, s)$ um círculo $C^{1}$ por partes em posição geral com $Y$. Assuma que $C$ tenha uma tangência interna com $Y$ no ponto q. Então olhando para as trajetórias de $Y$ ao redor de $q$ vemos que deve existir subintervalos fechados $[p, q]_{C} \subset C$ e $[q, r]_{C} \subset C$, com $[p, q]_{C} \cap[q, r]_{C}=\{q\}$ e um aplicação sobrejetora, contínua, revertendo orientação $T:[p, q]_{C} \rightarrow$ $[q, r]_{C}$ induzida pelo semifluxo positivo associado com $\left.Y\right|_{\mathbb{R}^{2} \backslash \bar{D}_{s_{0}}}$ com as seguintes propriedades

P.1 Para cada $z \in(p, q)$, existe um arco de trajetória $[z, T(z)] \subset \mathbb{R}^{2} \backslash D(C)$ de $Y$ que intercepta $C$ transversalmente e precisamente em $\{z, T(z)\}$.

P.2 A família $\{[z, T(z)]: z \in(p, q)\}$ depende continuamente de z e tende ao conjunto unitário $\{q\}$ quando $z \rightarrow q$.

Lema 2.5.3 Seja $s \geq s_{0}$ e $C \in \mathscr{G} \mathscr{P}(Y, s)$ um círculo $C^{1}$ por parte em posição geral com $Y$. Assuma que $C$ tenha uma tangência interna com $Y$ no ponto $q$. Se $[p, q]_{C} \subset C$ é maximal com relação à propriedade P.1 da Observação 2.5.2 então

(i) A semitrajetória positiva $\gamma_{p}^{+}$se iniciando em $p$ contém um arco de trajetória $[p, r]$ de $Y$ que encontra $C$ precisamente em $\{p, r\}$;

(ii) Cé transversal a $[p, r]$ em um de seus extremos e tem um tangência externa no outro extremo;

(iii) Seja $\Gamma=[p, r]_{C} \cup[p, r]$. Se r (resp. p) é a tangência externa então $\bar{D}(\Gamma)$ está contido em $\mathbb{R}^{2} \backslash D(C)$ e os pontos $\gamma_{p}^{+} \backslash[p, r]$ numa vizinhança de $r$ (resp. $p$ ) não pertencem a $\bar{D}(\Gamma)$.

Demonstração: (i) Primeiramente mostramos que $\gamma_{p}^{+} \cap C \supsetneq\{p\}$. Assuma o contrário, isto é, que $\gamma_{p}^{+} \cap C=\{p\}$. Então ou $\gamma_{p}^{+} \subset \bar{D}(C)$ ou $\gamma_{p}^{+} \subset \mathbb{R}^{2} \backslash D(C)$. Pela Propriedade P.1 não é difícil ver que $\gamma_{p}^{+} \subset \mathbb{R}^{2} \backslash D(C)$. Por (6) do Teorema 2.2.9 não exitem nem singularidades nem trajetórias periódicas em $\mathbb{R}^{2} \backslash D(C) \subset \mathbb{R}^{2} \backslash \bar{D}_{s_{0}}$. Agora seja $r \in C$ o único ponto satisfazendo $[q, r)=T((p, q])$ e seja $\gamma_{r}^{-}$ qualquer semitrajetória negativa de $Y$ se iniciando em $r$. Vamos mostrar que $\alpha\left(\gamma_{r}^{-}\right)=\infty$. Assuma por contradição que existe algum círculo $C_{1} \operatorname{com} C \subset D\left(C_{1}\right)$ e $\gamma_{r}^{-} \subset D\left(C_{1}\right)$. Mais uma vez, pelo Lema 2.2.4, como $\bar{D}\left(C_{1}\right) \backslash D(C)$ é uma região sem singularidades e trajetórias periódicas, e como todas tangências de $C \operatorname{com} Y$ são internas ou externas ( $C$ está em posição geral), temos que $\gamma_{r}^{-}$tem que cruzar $C$ transvesalmente em algum ponto $r_{1} \neq r$. Tome agora $z_{n} \rightarrow p, z_{n} \in(p, q]$. Segue-se 
da hipótese de maximalidade de $[p, q]_{C}$, que a seguência de arcos de trajetória $\left\{\left[z_{n}, T\left(z_{n}\right)\right]\right\}$ de $Y$ se acumula na trajetória positiva $\left[r_{1}, r\right]$ de $Y$. Assim para $n$ grande o suficiente $\left[z_{n}, T\left(z_{n}\right)\right] \cap C \supsetneq$ $\left\{z_{n}, T\left(z_{n}\right)\right\}$, o que contradiz P.1. Desta forma, $\alpha\left(\gamma_{r}^{-}\right)=\infty$. Não é difícil ver que $\gamma_{p}^{+}$e $\gamma_{r}^{-}$formam a fronteira de um pseudo setor hiperbólico, mesmo no caso em que $p=r$. Esta contradição com o Teorema2.4.2 prova (i). Item (ii) segue da maximalidade de $[p, q]_{C}$. A prova de (iii) é a mesma que a do Lema 2 em [27].

Lema 2.5.4 Seja $s \geq s_{0} e C \in \mathscr{G} \mathscr{P}(Y, s)$ um círculo $C^{1}$ por partes em posição geral com $Y$. Assuma que $C$ tenha uma tangência interna com $Y$ no ponto q. Mantenha toda a notação do Lema 2.5.3. Então existe $\widetilde{r} \in C$ arbitrariamente próximo de $r$ tal que o subintervalo $[p, \widetilde{r}]_{C}$ de $C$ contém o subintervalo $[p, r]_{C} \subset C$, e o seguinte vale

(i) podemos deformar o círculo $C$ em um novo círculo $C_{1} \in \mathscr{G} \mathscr{P}(Y, s)$ de tal maneira que a deformação fixa $C \backslash(p, \widetilde{r})_{C}$ e leva $[p, \widetilde{r}]_{C} \subset C$ em um intervalo $[p, \widetilde{r}]_{C_{1}} \subset C_{1}$ transversal $a Y, e$ assim livre de tangências com $Y$, que é próximo ao arco de trajetória $[p, r]$ de $Y$, ver Figura 5;

(ii) O número de tangências internas de $C_{1} \operatorname{com} Y$ é estritamente menor que aquele de $C$.

Demonstração: (i) Seja $\gamma_{p}^{+}$uma semitrajetória positiva de $\left.Y\right|_{\mathbb{R}^{2} \backslash \bar{D}_{s}}$ se iniciando em $p$ e seja $\Sigma_{2}$ uma seção transversal local a $Y$ passando por $z_{2} \in \gamma_{p}^{+} \backslash[p, r]$, onde $[p, r]$ é o (único) arco de trajetória de $\left.Y\right|_{\mathbb{R}^{2} \backslash \bar{D}_{s}}$ que se inicia em $p$ e termina em $r$. Pelo Teorema 2.3.2, podemos escolher algum campo vetorial $Y_{\lambda}: \mathbb{R}^{2} \backslash \bar{D}_{s} \rightarrow \mathbb{R}^{2}$, transversal a $\left.Y\right|_{\mathbb{R}^{2} \backslash \bar{D}_{s}}$, e algum arco de trajetória $\Delta$ de $Y_{\lambda}$ que parte de $p$, e termina em $\widetilde{z}_{2} \in \Sigma_{2} \backslash\left\{z_{2}\right\}$ e é próximo ao arco de trajetória $\left.\left[p, z_{2}\right] \subset \gamma_{p}^{+} \operatorname{de} Y\right|_{\mathbb{R}^{2} \backslash \bar{D}_{s}}$.

Além disto, com um ajuste de $Y_{\lambda}$, podemos assumir $\widetilde{z}_{2}$ arbitrariamente próximo a $z_{2}$ e em quaisquer das duas componentes conexas de $\Sigma_{2} \backslash\left\{z_{2}\right\}$. Assim, tomando $\widetilde{z}_{2}$ na componente conexa apropriada de $\Sigma_{2}$, temos que o correspondente arco de trajetória $\Delta=\left[p, \widetilde{z_{2}}\right]$ de $Y_{\lambda}$ intercepta $C$ em algum ponto $\widetilde{r}$ próximo a $r$ e de tal maneira que $[p, r]_{C} \subset[p, \widetilde{r})_{C}$. O subarco de trajetória $[p, \widetilde{r}] \subset \Delta$ de $Y_{\lambda}$ tem todas as propriedades requeridas. Substituindo $[p, \widetilde{r}]_{C}$ em $C$ por $[p, \tilde{r}] \subset \Delta$ obtemos um círculo $C_{1}$; (ii) Apenas observe que nos pontos de colagem $p$ e $\tilde{r}$ de $C \backslash(p, \tilde{r})_{C} \operatorname{com}[p, \tilde{r}] \subset \Delta$ o campo vetorial $Y$ é ainda transversal a $C_{1}$. Portanto, a deformação substitui o intervalo $[p, r]_{C}$ pelo segmento $[p, \widetilde{r}] \subset \Delta$, que elimina pelo menos duas tangências internas de $C \operatorname{com} Y$ deixando a outra inalterada.

Teorema 2.5.5 Para cada $s \geq s_{0}$, existe um círculo $C^{1}$ transversal a $Y$ contido em $\mathbb{R}^{2} \backslash \bar{D}_{s}$. 


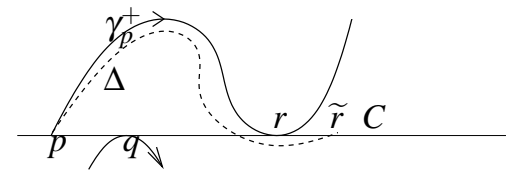

(a)

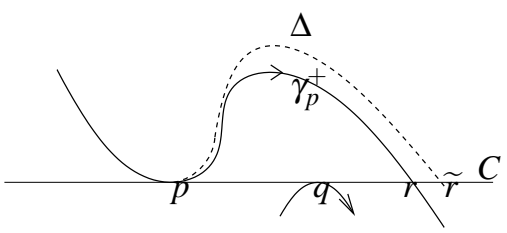

(b)

Figura 2.5: Seção transversal a um semifluxo positivo

Demonstração: Tome um círculo $C \in \mathscr{G} \mathscr{P}(Y, s)$. Como $C$ tem um número finito de tangências internas com $Y$, ao aplicar Lema 2.5.4 um número finito de vezes, podemos obter um círculo $\widetilde{C} \in \mathscr{G} \mathscr{P}(Y, s)$ com um número finito de tangências, todas externas. Denote por $\operatorname{deg}\left(\left.Y\right|_{\widetilde{C}}\right)$ o grau de Brower da aplicação $\left.Y\right|_{\widetilde{C}}$. Pelo Teorema 2.2.9, a aplicação $\left.Y\right|_{\widetilde{C}}$ é injetiva e preserva orientação; isto implica que $\operatorname{deg}\left(\left.Y\right|_{\widetilde{C}}\right)=1$. Por outro lado, como $\widetilde{C} \in \mathscr{G} \mathscr{P}(Y, s)$, temos que

$$
\operatorname{deg}\left(\left.Y\right|_{\widetilde{C}}\right)=\frac{2-n_{e}(Y, \widetilde{C})+n_{i}(Y, \widetilde{C})}{2}
$$

onde $n_{e}(Y, \widetilde{C})$ (resp. $\left.n_{i}(Y, \widetilde{C})\right)$ é o número de tangências externas (resp. internas) de $\widetilde{C}$ como $Y$ (ver [32, Teoremas 9.1 and 9.2, p. 166-174]).

Como $n_{i}(Y, \widetilde{C})=0$, fórmula (2.6) implica que $n_{e}(Y, \widetilde{C})=n_{i}(Y, \widetilde{C})=0$. Observando que $\widetilde{C}$ é um círculo $C^{1}$ por partes transversal a $Y$, podemos deformá-lo em um círculo $C^{1} \in \mathscr{G} \mathscr{P}(Y, s)$ transversal a $Y$.

\subsection{Estabilidade Assintótica no Infinito}

Nesta seção provamos o teorema principal. No que segue, $X \in \mathscr{D}_{\sigma}$ é um campo vetorial diferenciável e $Y: \mathbb{R}^{2} \rightarrow \mathbb{R}^{2}$ é o campo vetorial associado com $X$ dado pelo Teorema 2.2.9. O vetor constante $v$ é como no Teorema 2.2.9.

Lema 2.6.1 O ponto $\infty$ é um atrator ou repulsor de $Y$.

Demonstração: Pelo Teorema 2.5.5, existe uma família encaixada $\left\{\Gamma_{n} \subset \mathbb{R}^{2} \backslash \bar{D}_{s_{0}}: n \in \mathbb{N}\right\}$ de $C^{1}$ círculos transversais a $Y$ tendendo a infinito. Denote por $A_{n}=\bar{D}\left(\Gamma_{n}\right) \backslash D\left(\Gamma_{n-1}\right)$ a sequência correspondente de ânulos. Pelo item (6) do Teorema 2.2.9, não há nem singularidades nem trajetórias periódicas em $A_{n}$ de maneira que pelo Lema 2.2.4 nenhuma trajetória de $Y$ tem pontos de 
acumulação em $A_{n}$, para todo $n \in \mathbb{N}$. Isto implica que as trajetórias de $Y$ que interceptam $\Gamma_{1}$ têm que cruzar todos os círculos $\Gamma_{n}$. É claro que sob estas condições $\infty$ é ou um atrator ou um repulsor $Y$.

Teorema 2.6.2 O ponto no infinito de $\mathbb{R}^{2} \cup\{\infty\}$ é um atrator ou repulsor de $X+v$. Mais especificamente, se $\mathscr{I}(X) e ́<0(\geq 0)$, então $\infty$ é um repulsor (resp. um atrator) do campo vetorial $X+v$.

Demonstração: Que $\infty$ é um atrator ou repulsor de $X+v$ segue diretamente do lema anterior pois $Y$ e $X+v$ coincidem ao redor do infinito. Para terminar a prova note que $\mathscr{I}(X)=\mathscr{I}(X+$ $v)=\mathscr{I}(Y)$. Agora procedemos como em [28]. Assuma que $\infty$ seja um repulsor de $X+v$. Tome um círculo $C^{1} C \subset \mathbb{R}^{2} \backslash \bar{D}_{s}$ transversal a $Y$ tal que $\left.Y\right|_{C}$ aponta para dentro do disco $D(C)$ limitado por $C$. Pela Formula de Green $\int_{D(C)} \operatorname{Traço}(D Y)<0$. Por outro lado, por (4) do Teorema 2.2.9, $\int_{\mathbb{R}^{2} \backslash D(C)} \operatorname{Traço}(D Y)<0$. Assim,

$$
\begin{aligned}
\mathscr{I}(X) & =\mathscr{I}(Y)=\int_{\mathbb{R}^{2}} \operatorname{Traço}(D Y) d x \wedge d y= \\
& =\int_{\bar{D}(C)} \operatorname{Traço}(D Y) d x \wedge d y+\int_{\mathbb{R}^{2} \backslash \bar{D}(C)} \operatorname{Traço}(D Y) d x \wedge d y<0 .
\end{aligned}
$$

Portanto, se $\mathscr{I}(X) \geq 0$ então $\infty$ é um atrator de $X+v$. A prova do outro caso é similar.

Agora provamos nosso teorema principal.

Teorema D. Seja $X: \mathbb{R}^{2} \backslash \bar{D}_{\sigma} \rightarrow \mathbb{R}^{2}$ um campo vetorial diferenciável (mas não necessariamente $\left.C^{1}\right)$. Se para algum $\varepsilon>0, \operatorname{Spec}(X)$ é disjunto de $(-\varepsilon, 0] \cup\{z \in \mathbb{C}: \mathscr{R}(z) \geq 0\}$, então

a) Para todo $p \in \mathbb{R}^{2} \backslash \bar{D}_{\sigma}$, há uma única semitrajetória positiva de $X$ se iniciando em $p$;

b) $\mathscr{I}(X)$, o índice de $X$ no infinito, é um número bem definido da reta real estendida $[-\infty, \infty)$;

c) Existe um vetor constante $v \in \mathbb{R}^{2}$ tal que se $\mathscr{I}(X) e ́<0$ (resp. $\geq 0$ ), então o ponto no infinito da esfera de Riemann $\mathbb{R}^{2} \cup\{\infty\}$ é um repulsor (resp. um atrator) do campo vetorial $X+v$.

Demonstração: Temos que $X \in \mathscr{D}_{\sigma}$ de maneira que pelo Lema 2.2.6, $X \in \mathscr{D}\left(\mathbb{R}^{2} \backslash \bar{D}_{\sigma}\right)$. A prova de (a) segue pela aplicação do Teorema 2.2.3. As provas de b) e c) seguem do Corolário 2.2.12 e do Teorema 2.6.2, respectivamente. 


\section{Referências Bibliográficas}

[1] F. Abdenur, A. Avila, and J. Bochi. Robust transitivity and topological mixing for $C^{1}$-flows. Proc. Amer. Math. Soc., 132(3):699-705 (electronic), 2004.

[2] B. Alarcón, V. Guiñéz, and C. Gutierrez. Hopf bifurcation at infinity for planar vector fields. Accepted for publication in Disc. Cont. Dyn. Sys.

[3] S. K. Aranson, G. R. Belitsky, and E. V. Zhuzhoma. Introduction to the qualitative theory of dynamical systems on surfaces, volume 153 of Translations of Mathematical Monographs. American Mathematical Society, Providence, RI, 1996. Translated from the Russian manuscript by H. H. McFaden.

[4] S. K. Aranson and E. V. Zhuzhoma. On the $C^{r}$-closing lemma and the Koebe-Morse coding of geodesics on surfaces. J. Dynam. Control Systems, 7(1):15-48, 2001.

[5] S. R. Bernfeld. Liapunov functions and global existence without uniqueness. Proc. Amer. Math. Soc., 25:571-577, 1970.

[6] C. Bonatti and S. Crovisier. Récurrence et généricité. Invent. Math., 158(1):33-104, 2004.

[7] C. R. Carrol. Rokhlin towers and $C^{r}$ closing for flows on $\mathbf{T}^{2}$. Ergod. Th. Dynam. Sys., 12(1):683-706, 1992.

[8] T. M. Cherry. Topological Properties of the Solutions of Ordinary Differential Equations. Amer. J. Math., 59(4):957-982, 1937.

[9] C. Chicone. Ordinary differential equations with applications, volume 34 of Texts in Applied Mathematics. Springer-Verlag, New York, 1999.

[10] K. Ciesielski. The Poincaré-Bendixson theorems for two-dimensional semiflows. Topol. Methods Nonlinear Anal., 3(1):163-178, 1994. 
[11] M. Cobo, C. Gutierrez, and J. Llibre. On the injectivity of $C^{1}$ maps of the real plane. Canad. J. Math., 54(6):1187-1201, 2002.

[12] J. de Vries. Elements of topological dynamics, volume 257 of Mathematics and its Applications. Kluwer Academic Publishers Group, Dordrecht, 1993.

[13] F. Dumortier and P. De Maesschalck. Topics on singularities and bifurcations of vector fields. In Normal forms, bifurcations and finiteness problems in differential equations, volume 137 of NATO Sci. Ser. II Math. Phys. Chem., pages 33-86. Kluwer Acad. Publ., Dordrecht, 2004.

[14] F. Dumortier, R. Roussarie, J. Sotomayor, and H. Zoladek. Bifurcations of planar vector fields, volume 1480 of Lecture Notes in Mathematics. Springer-Verlag, Berlin, 1991. Nilpotent singularities and Abelian integrals.

[15] A. Fernandes, C. Gutierrez, and R. Rabanal. On local diffeomorphisms of $\mathbf{R}^{n}$ that are injective. Qual. Theory Dyn. Syst., 4(2):255-262 (2004), 2003.

[16] A. Fernandes, C. Gutierrez, and R. Rabanal. Global asymptotic stability for differentiable vector fields of $\mathbb{R}^{2}$. J. Differential Equations, 206(2):470-482, 2004.

[17] R. Feßler. A proof of the two-dimensional Markus-Yamabe stability conjecture and a generalization. Ann. Polon. Math., 62(1):45-74, 1995.

[18] H. Giacomini and M. Grau. On the stability of limit cycles for planar differential systems. $J$. Differential Equations, 213(2):368-388, 2005.

[19] C. Gutierrez. Structural stability for flows on the torus with a cross-cap. Trans. Amer. Math. Soc., 241:311-320, 1978.

[20] C. Gutierrez. Smoothability of Cherry flows on two-manifolds, volume 1007 of Springer Lecture Notes in Mathematics. American Mathematical Society, Providence, RI, 1981. Geometric Dynamics, Proc. Rio de Janeiro.

[21] C. Gutierrez. Smoothing continuous flows on two-manifolds and recurrences. Ergodic Theory Dynam. Systems, 6(1):17-44, 1986.

[22] C. Gutierrez. A counter-example to a $C^{2}$-closing lemma. Erg. Theor. Dyn. Sys., 7:509-30, 1987.

[23] C. Gutierrez. A solution to the bidimensional global asymptotic stability conjecture. Ann. Inst. H. Poincaré Anal. Non Linéaire, 12(6):627-671, 1995. 
[24] C. Gutierrez. On $C^{r}$-closing for flows on 2-manifolds. Nonlinearity, 13(6):1883-1888, 2000.

[25] C. Gutierrez and B. Pires. On Peixoto's conjecture for flows on non-orientable 2-manifolds. Proc. Amer. Math. Soc., 133(4):1063-1074 (electronic), 2005.

[26] C. Gutierrez and R. Rabanal. Injectivity of differentiable maps $\mathbb{R}^{2} \rightarrow \mathbb{R}^{2}$ at infinity. Preprint.

[27] C. Gutierrez and A. Sarmiento. Injectivity of $C^{1}$ maps $\mathbb{R}^{2} \rightarrow \mathbb{R}^{2}$ at infinity and planar vector fields. Astérisque, (287):xviii, 89-102, 2003. Geometric methods in dynamics. II.

[28] C. Gutierrez and M. A. Teixeira. Asymptotic stability at infinity of planar vector fields. Bol. Soc. Brasil. Mat. (N.S.), 26(1):57-66, 1995.

[29] C. Gutierrez and N. Van Chau. On nonsingular polynomial maps of $\mathbb{R}^{2}$. Preprint.

[30] C. Gutierrez and N. Van Chau. A remark on an eigenvalue condition for the global injectivity of maps of $\mathbb{R}^{2}$. Notas do ICMC, 224, 2005.

[31] P. Hartman. On local homeomorphisms of Euclidean spaces. Bol. Soc. Mat. Mexicana (2), 5:220-241, 1960.

[32] P. Hartman. Ordinary differential equations. John Wiley \& Sons Inc., New York, 1964.

[33] S. Hayashi. Connecting invariant manifolds and the solution of the $C^{1}$ stability and $\Omega$-stability conjectures for flows. Ann. of Math. (2), 145(1):81-137, 1997.

[34] S. Hayashi. Correction to: "Connecting invariant manifolds and the solution of the $C^{1}$ stability and $\Omega$-stability conjectures for flows" [Ann. of Math. (2) 145 (1997), no. 1, 81-137; MR1432037 (98b:58096)]. Ann. of Math. (2), 150(1):353-356, 1999.

[35] S. Hayashi. A $C^{1}$ make or break lemma. Bol. Soc. Brasil. Mat. (N.S.), 31(3):337-350, 2000.

[36] M.-R. Herman. Différentiabilité optimale et contre-exemples à la fermeture en topologie $C^{\infty}$ des orbites récurrentes de flots hamiltoniens. C. R. Acad. Sci. Paris Sér. I Math., 313(1):49-51, 1991.

[37] M.-R. Herman. Exemples de flots hamiltoniens dont aucune perturbation en topologie $C^{\infty}$ n'a d'orbites périodiques sur un ouvert de surfaces d'énergies. C. R. Acad. Sci. Paris Sér. I Math., 312(13):989-994, 1991.

[38] M. D. Hirsch. Dense recurrence in area-preserving flows on surfaces. Nonlinearity, 12:1545$1553,1994$. 
[39] A. Katok. Invariant measures of flows on orientable surfaces. Dokl. Akad. Nauk SSSR, 211:775-778, 1973.

[40] A. Katok and B. Hasselblatt. Introduction to the modern theory of dynamical systems, volume 54 of Encyclopedia of Mathematics and its Applications. Cambridge University Press, Cambridge, 1995. With a supplementary chapter by Katok and Leonardo Mendoza.

[41] R. Mañé. An ergodic closing lemma. Ann. of Math. (2), 116(3):503-540, 1982.

[42] N. G. Markley. The Poincaré-Bendixson theorem for the Klein bottle. Trans. Amer. Math. Soc., 135:159-165, 1969.

[43] N. G. Markley. On the number of recurrent orbit closures. Proc. Amer. Math. Soc., 25:413416, 1970.

[44] M. Martens, S. van Strien, W. de Melo, and P. Mendes. On Cherry flows. Ergodic Theory Dynam. Systems, 10(3):531-554, 1990.

[45] I. Nikolaev and E. Zhuzhoma. Flows on 2-dimensional manifolds, volume 1705 of Lecture Notes in Mathematics. Springer-Verlag, Berlin, 1999. An overview.

[46] C. Olech. On the global stability of an autonomous system on the plane. Contributions to Differential Equations, 1:389-400, 1963.

[47] C. Olech. Global phase-portrait of a plane autonomous system. Ann. Inst. Fourier (Grenoble), 14(fasc. 1):87-97, 1964.

[48] F. Oliveira. On the generic existence of homoclinic points. Ergodic Theory Dynam. Systems, 7(4):567-595, 1987.

[49] F. Oliveira. On the $C^{\infty}$ genericity of homoclinic orbits. Nonlinearity, 13(3):653-662, 2000.

[50] J. Palis, Jr. and W. de Melo. Geometric theory of dynamical systems. Springer-Verlag, New York, 1982. An introduction, Translated from the Portuguese by A. K. Manning.

[51] M. M. Peixoto. Structural stability on two-dimensional manifolds. Topology, 1:101-120, 1962.

[52] M. M. Peixoto. On a classification of flows on 2-manifolds. Proc. Symp. Dyn. Syst. Salvador, 1973.

[53] W. F. Pfeffer. The multidimensional fundamental theorem of calculus. J. Austral. Math. Soc. Ser. A, 43(2):143-170, 1987. 
[54] C. C. Pugh. An improved closing lemma and a general density theorem. Amer. J. Math., 89:1010-1021, 1967.

[55] C. C. Pugh. Against the $C^{2}$ closing lemma. J. Differential Equations, 17:435-443, 1975.

[56] C. C. Pugh. A special $C^{r}$ closing lemma. In Geometric dynamics (Rio de Janeiro, 1981), volume 1007 of Lecture Notes in Math., pages 636-650. Springer, Berlin, 1983.

[57] C. C. Pugh. The $C^{1}$ connecting lemma. J. Dynam. Differential Equations, 4(4):545-553, 1992.

[58] C. C. Pugh and C. Robinson. The $C^{1}$ closing lemma, including Hamiltonians. Ergodic Theory Dynam. Systems, 3(2):261-313, 1983.

[59] R. Roussarie. Bifurcation of planar vector fields and Hilbert's sixteenth problem, volume 164 of Progress in Mathematics. Birkhäuser Verlag, Basel, 1998.

[60] A. J. Schwartz. A generalization of a Poincaré-Bendixson theorem to closed two-dimensional manifolds. Amer. J. Math. 85 (1963), 453-458; errata, ibid, 85:753, 1963.

[61] A. Strauss and J. A. Yorke. On the fundamental theory of differential equations. SIAM Rev., 11:236-246, 1969.

[62] F. Takens. Singularities of vector fields. Inst. Hautes Études Sci. Publ. Math., (43):47-100, 1974. 